\title{
THE IN VITRO EFFECTS OF PLATELET PRODUCTS ON THE BIOPHYSIOLOGICAL FUNCTIONS OF HUMAN BONE MARROW MESENCHYMAL STROMAL CELLS: A SYSTEMATIC REVIEW
}

\author{
J. Vun ${ }^{1,2,3, *}$, M. Panteli ${ }^{1,2,3}$, E. Jones ${ }^{2}$ and P.V. Giannoudis ${ }^{1,2,3,4}$ \\ ${ }^{1}$ Academic Department of Trauma and Orthopaedics, School of Medicine, University of Leeds, Leeds, UK \\ ${ }^{2}$ Leeds Institute of Rheumatic and Musculoskeletal Medicine, University of Leeds, Leeds, UK \\ ${ }^{3}$ Leeds Orthopaedic and Trauma Sciences, Leeds General Infirmary, University of Leeds, Leeds, UK \\ ${ }^{4}$ NIHR Leeds Biomedical Research Centre, Chapel Allerton Hospital, Leeds, UK
}

\begin{abstract}
Platelet products (PP) and bone-marrow aspirate are popular sources of osteoinductive signalling molecules and osteogenic bone marrow mesenchymal stromal cells (BM-MSCs) used in the treatment of impaired bone healing. However, the combined use of PP and BM-MSCs in clinical studies has reported mixed results. Understanding the cellular and molecular interactions between PP and BM-MSCs plays the important role of guiding future research and clinical application. This systematic review investigates the effects of PP on the biophysiological functions of BM-MSCs in in vitro human studies, including (i) proliferation, (ii) migration, (iii) differentiation, (iv) growth factor/cytokine/protein expression, (v) immunomodulation, (vi) chemotactic effect on haematopoietic stem cells, (vii) response to apoptotic stress, and (viii) gene expression. In vitro studies in human have demonstrated the multi-faceted 'priming effect' of PP on the biophysiological functions of BM-MSCs. PP has been shown to improve proliferation, migration, osteogenic differentiation, reaction to apoptotic stress as well as immunomodulatory, pro-angiogenic and pro-inflammatory capacities of BMMSCs. Several factors are highlighted that restrict the transferability of these findings into clinical practice. Therefore, more collaborative in vitro research in humans modelled to reflect clinical practice is required to better understand the effects of PP exposure on the biophysiological function(s) of BM-MSCs in human.
\end{abstract}

Keywords: Bone marrow mesenchymal stem cell(s), mesenchymal stromal cell(s), platelet-rich plasma, platelet lysate, platelet concentrate, platelet releasate, platelet gel, platelet-rich fibrin, bone healing, non-union.

*Address for correspondence: Dr James Shen Hwa Vun, Clinical Research Fellow, Academic Department of Trauma and Orthopaedics, School of Medicine, University of Leeds, Clarendon Wing, Level A, Great George Street, Leeds, LS1 3EX, West Yorkshire, UK.

Telephone number: +44 1133922750 Fax number: +44 113392329 Email: j.vun@nhs.net

Copyright policy: This article is distributed in accordance with Creative Commons Attribution Licence (http://creativecommons.org/licenses/by-sa/4.0/).

\begin{tabular}{llll}
\hline & List of Abbreviations & BMP & bone morphogenetic protein \\
& & CEPBA & CCAAT/enhancer-binding protein $\alpha$ \\
aMEM & alpha-minimum essential medium & CFU & colony-forming unit \\
conditioned medium & CM & collagen type 1 \\
ACAN & activated PRP & COL1 & collagen type $1 \alpha 1$ \\
ACD-A & acid-citrate dextrose solution A & COL1A1 & collagen type 2 1 \\
ADIPOQ & adiponectin, C1Q and collagen & COL2A1 & cartilage oligomeric matrix protein \\
& domain containing & COMP & cyclooxygenase-2 \\
aFGF & acidic fibroblast growth factor & COX-2 & citrate phosphate dextrose \\
ALP & alkaline phosphatase & CPD & 4',6-diamidino-2-phenylindole \\
APOE & apolipoprotein E & DAPI & Dulbecco's modified Eagle's medium \\
bFGF & basic fibroblast growth factor & DMEM & ethylenediamine tetraacetic acid \\
BMA & bone marrow aspirate & EDTA & fatty acid binding protein 4 \\
BMAC & BMA concentrate & FABP4 & foetal bovine serum \\
BM-MSC & bone marrow mesenchymal stromal & FBS & filtered PL
\end{tabular}




\begin{tabular}{|c|c|c|c|}
\hline FTPL & frozen-thawed PL & RUNX2 & runt-related transcription factor 2 \\
\hline \multirow[t]{2}{*}{ G-CSF } & granulocyte colony-stimulating & SDF-1 & stroma-derived factor-1 \\
\hline & factor & SEM & scanning electron microscopy \\
\hline GLUT4 & glucose transporter type 4 & SNPL & PL obtained by sonification \\
\hline HG-DMEM & high-glucose DMEM & SOX & SRY-box transcription factor \\
\hline HGF & hepatocyte growth factor & SPARC & secreted protein acidic and cysteine \\
\hline HPLF & human platelet lysate from fresh & & rich \\
\hline \multirow{3}{*}{ HPLO } & platelet concentrates & SREBP1 & sterol regulatory element-binding \\
\hline & human platelet lysate from expired & & transcription factor 1 \\
\hline & platelet concentrates & TERT & telomerase reverse transcriptase \\
\hline HSCs & haematopoietic stem cells & TGF & transforming growth factor \\
\hline IL & interleukin & ucPRP & umbilical cord PRP \\
\hline IP-10 & interferon- $\gamma$-inducible protein 10 & uPA & urokinase-type plasminogen \\
\hline iNOS & inducible nitric oxide synthase & & activator \\
\hline ITS & insulin-transferrin-selenium & VEGF & vascular endothelial growth factor \\
\hline KO-DMEM & knock out-DMEM & & receptor \\
\hline L-PRP & leukocyte-rich PRP & & \\
\hline
\end{tabular}

\section{Introduction}

LG-DMEM low-glucose DMEM

LPL

$\mathrm{LXR} \alpha$

MCP-1

MMP

$\mathrm{MNC}$

MIP

naPPP

naPRP

$N F-\kappa B$

NM

$\mathrm{NO}$

$\mathrm{OM}$

PAI-1

PBMC

PD

PDGF

PG

PG-CM

PGE2

PL

PLGF

PLIN

POU5F1

PP

lipoprotein lipase

liver $X$ receptor alpha

monocyte chemoattractant protein-1

matrix metalloproteinases

mononuclear cell

macrophage inflammatory protein

non-activated PPP

non-activated PRP

nuclear factor kappa-light-chain-

enhancer of activated B cells

nutrient medium

nitric oxide

osteogenic differentiation medium

plasminogen activator inhibitor-1

peripheral mononuclear cells

population doubling

platelet-derived growth factor

platelet gel

platelet gel conditioned-medium

prostaglandin E2

platelet lysate

placental growth factor

perlipin

PPARG

POU class 5 homeobox 1

platelet product

PPP $\quad$ receptor gamma

P-PRP leukocyte-poor PRP

PRISMA Preferred Reporting Items for

Systematic Reviews and Meta-

Analyses

PR

platelet releasate

PRC platelet-rich concentrate

PRGF plasma rich in growth factors

PRF platelet-rich fibrin

PRP platelet-rich plasma

PRP-CM PRP-conditioned culture medium

PTGES prostaglandin E synthase

RANTES regulated on activation, normal $\mathrm{T}$

cell expressed and secreted

Bone healing is a unique biological process leading to bone repair and restoration of bone function to its pre-injury level (Einhorn and Gerstenfeld, 2015). According to the 'diamond concept', bone healing involves the orchestrated interaction between multiple factors at the molecular, physiological and biomechanical level (Giannoudis et al., 2007).

The three fundamental molecular components of bone healing are: (a) osteogenic progenitor cells, such as BM-MSCs; (b) osteoinductive signalling molecules, such as growth factors and cytokines; (c) osteoconductive scaffold/matrix (Calori and Giannoudis, 2011). In addition, other essential factors at the physiological and biomechanical levels crucial to successful bone healing include the host (i.e. patient) physiology, mechanical stability and vascularity of the affected local bone environment (Giannoudis et al., 2008).

Although every attempt has been made to optimise the physiological and biomechanical factors during primary surgery, impaired bone healing (e.g. delayed union and non-union) remains common, occurring in 5-10\% of all acute fractures (Ekegren et al., 2018; Mills and Simpson, 2013). Impaired bone healing is challenging to treat and poses a significant burden on the patient, socioeconomic and healthcare systems (Bishop et al., 2012; Hak et al., 2014). Multiple operations are often required to achieve treatment success, which exposes patient to further risks of peri-operative complications, prolonged periods of rehabilitation and delay in returning to work. From a socioeconomic perspective, the direct treatment costs have been estimated to be between $£ 7,000$ and $£ 79,000$ per person depending on complexity (Bishop et al., 2012). In European healthcare systems the indirect costs through productivity losses have been estimated to be 10 times higher (Hak et al., 2014).

In an effort to optimise bone healing, surgeons have recently attempted a 'polytherapy' approach involving the simultaneous application of all the 
three fundamental molecular components of bone healing, as mentioned previously (Calori et al., 2011). Autologous sources of these biological components, which guarantees histocompatibility, minimal morbidity and ease of access during the harvesting process, are ultimately desirable. This has made autologous iliac crest bone graft, PPs and BMA/ BMAC popular sources of osteoconductive scaffolds, osteoinductive molecules and osteogenic BM-MSCs, respectively (Calori and Giannoudis, 2011; Gianakos et al., 2017; Roffi et al., 2017).

PPs or platelet derivatives are terminologies often used interchangeably to represent haemoderivatives containing platelets and growth factors, cytokines and molecules beneficial for bone healing. PPs, which are commonly used in the clinical and laboratory settings, include PRP, PL, PRC, PR, PG and PRF.

Briefly, when harvested in the presence of anticoagulants, peripheral blood can be centrifuged to produce two distinct fractions: (i) PRP (also referred to as platelet concentrates) and (ii) PPP. On the other hand, centrifugation of blood harvested in the absence of anticoagulants induces coagulation, producing PRF. Finally, when peripheral blood is allowed to naturally coagulate and then is centrifuged, this produces autologous serum.

Inducing the release of growth factors from PRP ex vivo could be achieved through two common methods: (i) physical disruption/lysis and (ii) chemical activation. Physical disruption of PRP, such as repeated freeze-thaw cycles and ultrasound sonification, results in platelet lysis and, therefore, acellular PL rich in growth factors. Chemical activation of PRP (commonly with calcium salts or thrombin) leads to the production of two distinct products, due to two distinct processes that occur during activation: (i) platelet degranulation and the release of a plasmatic fraction rich in growth factors, known as platelet releasate or PRGF or platelet released supernatants; (ii) fibrinogenesis leading to formation of a platelet-rich clot, also known as PG (Soares et al., 2020).

Several classification systems for platelet concentrates and PRP have been developed in the last decade (DeLong et al., 2012; Dohan Ehrenfest et al., 2009; Lana et al., 2017; Magalon et al., 2016; Mautner et al., 2015; Mishra et al., 2012). The strengths of these classifications lie in their call for a comprehensive documentation of centrifugation and preparation protocol, cellular constituents (platelet concentration, red blood cell count, white blood cell count) and method of platelet activation. However, the choice of using the term PRP for PP that have undergone exogenous chemical activation (which strictly speaking should be called PG) in these classification systems creates further confusion. Consequently, PRP is often used loosely as a universal term encompassing all forms of platelet-rich preparations with variable preparation protocols and composition. Therefore, there still remains a lack in standardised terminology that allows for the effective, accurate description of these PPs, reflective of its contents and physical properties (e.g. liquid, gel, fibrin membrane).

Clinical studies investigating the use of PPs either alone or in combination with BM-MSCs have reported mixed results (Andia and Maffulli, 2019; Bielecki et al., 2008; Calori et al., 2008; Chiang et al., 2007; Etulain, 2018; Galasso et al., 2008; Golos et al., 2014; Malhotra et al., 2015; Mariconda et al., 2008; Roffi et al., 2017; Sanchez et al., 2009; Say et al., 2014; Verboket et al., 2018). The pooling of fractures from different anatomical sites (e.g. tibia, femur, humerus) (Bielecki et al., 2008; Calori et al., 2008; Chiang et al., 2007; Galasso et al., 2008; Golos et al., 2014; Malhotra et al., 2015; Mariconda et al., 2008; Roffi et al., 2017; Sanchez et al., 2009; Say et al., 2014), coupled with the heterogeneity in techniques used to harvest and prepare PPs and BM-MSCs (Etulain, 2018; Roffi et al., 2017) made comparison between studies difficult, hindering the in-depth scientific understanding of these biological therapies.

A systematic review on in vitro studies in human will provide an improved scientific understanding of how PPs interacts with and affects the biophysiological functions of BM-MSCs at the cellular level. This has far-reaching importance, serving to guide future research and the clinical application of BM-MSCs and PPs when treating patients with or at risk of impaired bone healing.

The main objective of the present systematic review was to investigate the biophysiological functions of BM-MSCs influenced by PPs. Additionally, the different methods to harvest, prepare and culture BM-MSCs and PPs were also evaluated.

\section{Materials and Methods}

\section{Protocol}

This systematic review was conducted in accordance with the key principles recommended in PRISMA statement (Hutton et al., 2015; Moher et al., 2009).

\section{Information sources and search strategy}

A comprehensive systematic bibliographic search was performed in the following databases on the $8^{\text {th }}$ of September 2020: PubMed, Medline (via Ovid), Embase (via Ovid), Scopus, Google Scholar and the Cochrane Library. No restriction was applied to the date of publication. The following search terms were used: 'bone' or 'bone marrow' or 'mesenchymal stem cell(s)' or 'mesenchymal stromal cell(s)' or 'MSC' or 'platelet product' or 'platelet rich plasma' or 'PRP' or 'platelet lysate' or 'platelet rich concentrate' or 'platelet releasate' or 'platelet rich fibrin' or 'platelet gel' or 'platelet released growth factors'. The choice of studies was limited to human studies only. Bibliographies of all identified studies were manually searched for any additional eligible studies. 


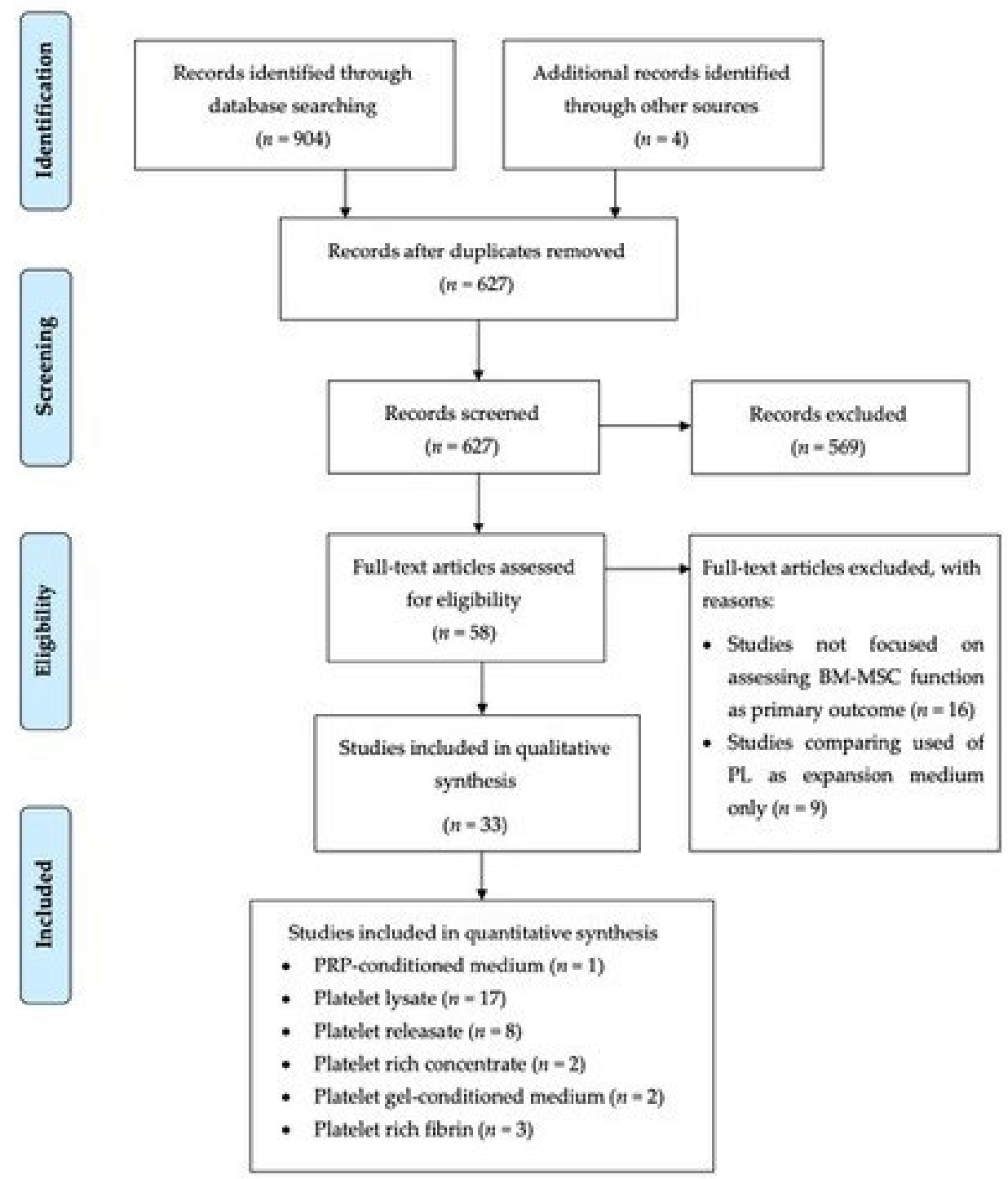

Figure 1. PRISMA flowchart on study selection.

\section{Eligibility criteria}

Studies were original research articles that fulfilled the following inclusion criteria: (i) in vitro studies in human; (ii) studies that included comparison between BM-MSCs versus PPs and BM-MSCs. Studies involving use of scaffold (in addition to PPs and BMMSCs) were excluded, since the presence of another osteoconductive \pm osteoinductive element (i.e. the scaffold itself) complicates the interpretation on how PP affects the biophysiological function of BM-MSCs. Furthermore, in vitro studies combining a xenogeneic component (PPs or BM-MSCs) with human BM-MSCs or PPs, clinical studies and case reports were also excluded.

\section{Study selection}

All retrieved records were first uploaded into EndNote followed by removal of duplicate records. Then, retrieved records were independently screened by two reviewers (JV, MP) in a blinded, standardised manner. Title and abstract sift was conducted first, followed by review of full text. Only studies fulfilling the eligibility criteria were included. Any discrepancies or disagreement between reviewers were resolved by consensus.

\section{Data extraction}

Relevant information on author, year of publication, patient demographics, site of harvest, processing methods (e.g. centrifugation), tissue characteristics, culture method, incubation period, laboratory assay, biophysiological functions of BM-MSCs were carefully extracted. The biophysiological functions of interest were proliferation, migration, differentiation, cytokine/protein/growth factor expression, gene expression, immunomodulation and reaction to apoptotic stress.

\section{Data analysis}

Outcomes of interest were entered into an electronic database. The following were compared across different studies: (i) harvesting process, (ii) centrifugation technique/preparatory steps, (iii) tissue culturing and incubation period of PPs and BM-MSCs, (iv) effect of PP exposure on the biophysiological functions of BM-MSCs. 


\section{Results}

\section{Study selection}

A total of 904 citations were identified during the searches, followed by removal of duplicate records $(n=281)$. At title and abstract sift, 569 citations not meeting the inclusion criteria were excluded. Full text sifting of the remaining citations $(n=58)$ excluded a further 25 citations, yielding a total of 33 citations for the final analysis (Fig. 1).

\section{Study characteristics}

Table 1 summarises the main study characteristics of the 33 studies included in the analyses and the BMMSC functions assessed. The choice of PPs used was as follows: PRP-CM $(n=1) ; \mathrm{PL}(n=17) ; \mathrm{PR}(n=8)$; PRC $(n=2)$; PG-CM $(n=2)$ and PRF $(n=3)$. The effect of PPs on the proliferation of BM-MSCs was most commonly assessed $(n=27)$, followed by differentiation $(n=23)$; gene expression $(n=14)$; migration $(n=8)$; cytokine/ growth factor release $(n=7)$ and immunomodulation $(n=6)$. Other functions less commonly assessed were the response of BM-MSCs towards apoptotic stress $(n=1)$ (Yin et al., 2016) and stimulation of fibrinolysis and plasminogen activity $(n=1)$ (Agis et al., 2009).

The majority of the studies (21 out of total 33) reported on the sample sizes for both PPs and BMMSCs. Only 6 of these 21 studies had a sample size of $n \geq 10$ for both components (PPs and BM-MSCs) (Ben Azouna et al., 2012; Jenhani et al., 2011; Lucarelli et al., 2003; Moisley et al., 2019; Verrier et al., 2010; Yin et al., 2016); with the remaining having a small sample size of $n \leq 6$ for one or both components. Discrepancy in sample size between PPs and BM-MSCs within the same study was also observed (Amable et al., 2014; Ben Azouna et al., 2012; Bernardi et al., 2017; Bernardi et al., 2013; Goedecke et al., 2011; Gottipamula et al., 2012; Infante et al., 2017; Jenhani et al., 2011; Kasten et al., 2008; Parsons et al., 2008; Perut et al., 2013; Prins et al., 2009; Vogel et al., 2006; Yin et al., 2016) (Table 2a,b and $3 \mathbf{a}, \mathbf{b})$.

\section{Harvesting process}

With the exception of two studies (Dohan Ehrenfest et al., 2010; Verrier et al., 2010), PPs and BM-MSCs used in most of these in vitro studies were harvested from different donors, explaining the discrepancy in sample size between PPs and BM-MSCs seen in some studies (Table 2a, b and $3 \mathbf{a}, \mathbf{b}$ ).

\section{PPS}

PRP-CM (Yin et al., 2016), PRC (Parsons et al., 2008; Samuel et al., 2016), PG-CM (Perut et al., 2013; Schar et al., 2015) and PRF (Dohan Ehrenfest et al., 2010; Lucarelli et al., 2010; Moradian et al., 2017) were all harvested from peripheral blood. For studies assessing PR, only half $(n=4)$ used PR processed from peripheral blood; whilst the rest were from platelet apheresis/concentrates. As for studies using PL, with the exception of 3 studies (peripheral blood: $n=2$; commercial grade PL: $n=1$ ), all other studies used PL processed using buffy coats or platelet apheresis/ concentrate acquired from a blood bank.

Only a small number of studies reported on harvest volume $(n=12)$. The health $(n=12)$ and age $(n=8)$ of volunteers/patients were only reported in some studies (Table 2a,b).

\section{BM-MSCs}

In most of the studies BM-MSCs were harvested from BMA $(n=20)$ and bone marrow harvested during surgery $(n=5)$. The remaining studies used either commercially purchased BM-MSCs $(n=4)$ or cryopreserved BM-MSCs $(n=4)$ (Table $3 \mathbf{a}, \mathbf{b})$.

The commonest harvest site was iliac crest $(n=11)$, followed by long bones $(n=3)$. Other sources of BM-MSCs include femoral head $(n=1)$ (Agis et al., 2009) and maxilla $(n=1)$ (Dohan Ehrenfest et al., 2010); whilst 17 studies did not specify harvest site of the BM-MSCs used. The volume of bone marrow harvested was only reported in 6 studies. Likewise, the age $(n=16)$ and health $(n=19)$ of volunteers/ patients were only reported in some studies.

\section{Preparation and processing of harvested samples} PPS - centrifugation, anticoagulant, platelet activation and concentrations of platelet and leukocytes

As illustrated in Table 4a-e, the centrifugation protocol was very variable among studies, most of which used a laboratory centrifugation system.

Similarly, the use of anticoagulants was variable and dependant on (i) protocol, (ii) source of platelet used (e.g. peripheral blood, platelet apheresis concentrate, commercial PL) and (iii) the choice of PPs intended for the experiment. Anticoagulants, such as ACD-A $(n=8)$, heparin $(n=3), \operatorname{CPD}(n=3)$ and sodium citrate $(n=1)$ were used if the first step involved producing PRP, before subjecting the sample to other processes, such as freeze-thawing or activation.

Activation of platelet to release the alpha-granules (Marx, 2004) was performed in studies assessing PR, PG-CM and PRF. Activation was achieved by exposing PPs to calcium chloride in 5 studies, to thrombin in 4 and to both thrombin and calcium gluconate in 1 study.

Leukocyte and platelet concentrations of PPs were not reported in most studies. Leukocyte concentration was only documented in 4 studies (Infante et al., 2017; Moisley et al., 2019; Perut et al., 2013; Yin et al., 2016), whereas platelet concentration was reported in more studies $(n=15)$. Only 3 studies compared the effects of leukocyte content on the biophysiological functions of BM-MSCs (Moisley et al., 2019; Perut et al., 2013; Yin et al., 2016).

BM-MSCs - centrifugation isolation method, fresh ws. subcultured cells

Most of the studies used a laboratory system to isolate mononuclear cells rich in BM-MSCs from the 
Table 1. Study characteristics. $\mathrm{O}=$ osteogenesis; $\mathrm{A}=$ adipogenesis; $\mathrm{C}=$ chondrogenesis.

\begin{tabular}{|c|c|c|c|c|c|c|c|}
\hline Author (year) & Proliferation & Differentiation & Migration & $\begin{array}{c}\text { Cytokine/ } \\
\text { protein } \\
\text { expression }\end{array}$ & $\begin{array}{c}\text { Gene } \\
\text { expression }\end{array}$ & Immunomodulation & Others \\
\hline \multicolumn{8}{|c|}{ PRP-CM } \\
\hline Yin et al., 2016 & $\checkmark$ & $\mathrm{O}$ & $\checkmark$ & $\checkmark$ & $\checkmark$ & $\checkmark$ & $\begin{array}{l}\text { Cell viability/ } \\
\text { apoptosis }\end{array}$ \\
\hline \multicolumn{8}{|c|}{ PL } \\
\hline $\begin{array}{l}\text { Karadjian } \\
\text { et al., } 2020\end{array}$ & $\checkmark$ & $\mathrm{O}$ & & & & & \\
\hline Moisley et al., 2019 & $\checkmark$ & & $\checkmark$ & & & & \\
\hline Skific et al., 2018 & $\checkmark$ & $\mathrm{O}$ and $\mathrm{A}$ & & & & & \\
\hline Infante et al., 2017 & $\checkmark$ & & $\checkmark$ & & $\checkmark$ & & \\
\hline $\begin{array}{l}\text { Muraglia } \\
\text { et al., } 2015\end{array}$ & $\checkmark$ & & & & & & \\
\hline $\begin{array}{c}\text { Muraglia } \\
\text { et al., 2014 } \\
\text { (lyophilised PL) }\end{array}$ & $\checkmark$ & & & & & & \\
\hline $\begin{array}{l}\text { Jonsdottir-Buch } \\
\text { et al., } 2013\end{array}$ & $\checkmark$ & $\mathrm{O}, \mathrm{A}$ and $\mathrm{C}$ & & & $\checkmark$ & $\checkmark$ & \\
\hline Bernardi et al., 2013 & $\checkmark$ & $\mathrm{O}, \mathrm{A}$ and $\mathrm{C}$ & & & & $\checkmark$ & \\
\hline Murphy et al., 2012 & $\checkmark$ & & $\checkmark$ & & & & \\
\hline Lange et al., 2012 & & $\mathrm{~A}$ & & & $\checkmark$ & ( & \\
\hline $\begin{array}{l}\text { Gottipamula } \\
\text { et al., } 2012\end{array}$ & $\checkmark$ & $\mathrm{O}, \mathrm{A}$ and $\mathrm{C}$ & & $\checkmark$ & $\checkmark$ & $\checkmark$ & \\
\hline $\begin{array}{l}\text { Ben Azouna } \\
\text { et al., } 2012\end{array}$ & $\checkmark$ & $\mathrm{O}, \mathrm{A}$ and $\mathrm{C}$ & & $\checkmark$ & $\checkmark$ & & \\
\hline Jenhani et al., 2011 & $\checkmark$ & & & $\checkmark$ & & & \\
\hline $\begin{array}{l}\text { Goedecke } \\
\text { et al., } 2011\end{array}$ & $\checkmark$ & $\mathrm{O}$ and $\mathrm{A}$ & $\checkmark$ & $\checkmark$ & & & \\
\hline Verrier et al., 2010 & & $\mathrm{O}$ & & $\checkmark$ & $\checkmark$ & & \\
\hline Prins et al., 2009 & $\checkmark$ & $\mathrm{O}, \mathrm{A}$ and $\mathrm{C}$ & & & & & \\
\hline Vogel et al., 2006 & & $\mathrm{O}, \mathrm{A}$ and $\mathrm{C}$ & & & & & \\
\hline \multicolumn{8}{|c|}{ PR } \\
\hline Nguyen et al., 2019 & $\checkmark$ & & $\checkmark$ & & & & \\
\hline Liou et al., 2018 & 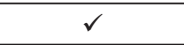 & $C$ & & & $\checkmark$ & & \\
\hline $\begin{array}{l}\text { Bernardi et al., } 2017 \\
\text { (assessed PR and } \\
\text { PL) }\end{array}$ & $\checkmark$ & $\mathrm{O}, \mathrm{A}$ and $\mathrm{C}$ & & & & $\checkmark$ & \\
\hline $\begin{array}{l}\text { Kosmacheva } \\
\text { et al., } 2014\end{array}$ & & $\mathrm{O}$ & & & $\checkmark$ & & \\
\hline Amable et al., 2014 & $\checkmark$ & $\mathrm{O}, \mathrm{A}$ and $\mathrm{C}$ & & $\checkmark$ & $\checkmark$ & & \\
\hline Agis et al., 2009 & & & & & & & $\begin{array}{l}\text { Stimulation of } \\
\text { fibrinolysis and } \\
\text { plasminogen } \\
\text { activity }\end{array}$ \\
\hline Gruber et al., 2004 & $\checkmark$ & $\mathrm{O}$ & $\checkmark$ & & $\checkmark$ & & \\
\hline Lucarelli et al., 2003 & $\checkmark$ & $\mathrm{O}$ & & & $\checkmark$ & & \\
\hline \multicolumn{8}{|c|}{ PRC } \\
\hline Samuel et al., 2016 & $\checkmark$ & $\mathrm{O}$ & & & $\checkmark$ & & \\
\hline Parsons et al., 2008 & $\checkmark$ & $\mathrm{O}$ & & & $\checkmark$ & & \\
\hline \multicolumn{8}{|c|}{ PG-CM } \\
\hline Schar et al., 2015 & & & $\checkmark$ & & & & \\
\hline Perut et al., 2013 & $\checkmark$ & $\mathrm{O}$ & & & & & \\
\hline \multicolumn{8}{|c|}{ PRF } \\
\hline $\begin{array}{l}\text { Moradian } \\
\text { et al., } 2017\end{array}$ & $\checkmark$ & & & & & & \\
\hline Lucarelli et al., 2010 & $\checkmark$ & & & & & & \\
\hline $\begin{array}{c}\text { Dohan Ehrenfest } \\
\text { et al., } 2010\end{array}$ & $\checkmark$ & $\mathrm{O}$ & & & & & \\
\hline
\end{tabular}


bone marrow harvested. This was achieved by using a density-gradient centrifugation system $(n=19)$, and laboratory-grade centrifugation into pellets and manual isolation by nucleated cell counting before seeding $(n=3) .5$ studies using clinically harvested bone marrow and 5 studies using commercially/tissue bank acquired BM-MSCs did not describe the method used to isolate mononuclear cells.

With the exception of 2 studies that strictly used freshly aspirated BM-MSCs only in all of their laboratory assays (Muraglia et al., 2014; Perut et al., 2013), the majority either used fresh BM-MSC in their proliferation assays only $(n=3)$ or subcultured BMMSCs (BM-MSCs transferred from previous culture into a new vessel for continued growth in fresh culture medium) $(n=31)$. Huge variation exists in tissue culture medium used for cultivation/expansion and the passage number of BM-MSCs used for assays (median: passage 3, range: passage 0 to 6) (Table 5a-c). Cellular detachment of subcultured BM-MSCs was performed by enzymatic methods (porcine trypsin, $n=22$; recombinant corn-derived enzyme TrypZean, $n=2)$.

\section{Incubation of BM-MSCs with PPs}

All studies were conducted by exposing BM-MSCs to a continuous dose of PPs. As previously mentioned, except for 2 donor-matched studies (Dohan Ehrenfest et al., 2010; Verrier et al., 2010), BM-MSCs and PPs used in all other in vitro studies were not harvested from the same donor(s). Most studies $(70.9 \%, n=22$ out of total 31) with more than one donor reported pooling PPs in experiments (Table 6a-c).

Incubation period of BM-MSCs with PPs was variable among studies and dependent on the physiological function of BM-MSCs assessed: (i) migration: 4 to $48 \mathrm{~h}$; (ii) proliferation: $2 \mathrm{~h}$ to $14 \mathrm{~d}$; (iii) differentiation: 8 to $21 \mathrm{~d}$; (iv) gene expression: 5 to $21 \mathrm{~d}$; (v) cytokine, protein, growth factor expression: 3 to $14 \mathrm{~d}$; (v) cell viability/apoptosis: $7 \mathrm{~d}$; (vi) fibrin dissolution assay: $24 \mathrm{~h}$ (Table 6a-c). The choices of tissue culture medium and seeding density used for each assay were reported in all studies. Notably, the choices of tissue culture medium, culture dish/ well plates and seeding density for assays were very variable among studies (Table 7a-c).

\section{Effects of PPs on proliferation of BM-MSCs}

Table 8a-c summarises the choice of laboratory assays, PPs used, comparative groups and their findings. The presence of PPs was found to significantly increase proliferation of BM-MSCs in most of the studies, when compared to controls: PRP-CM $(n=1)$, PL $(n=13), \mathrm{PR}(n=3), \mathrm{PRC}(n=1), \mathrm{PG}-\mathrm{CM}(n=1), \mathrm{PRF}$ $(n=2)$.

The effects of (i) concentration/dose of PPs, (ii) leukocyte content, and (iii) incubation time on proliferation of BM-MSCs have been assessed in some studies. In terms of concentrations of PPs in PP-conditioned medium, variable concentrations have been used, with $5 \%$ to $10 \%$ being the commonest. The increase in proliferation of BMMSCs demonstrated a positive correlation with PP concentrations up to $10 \%$. Only 2 studies assessed the effect of PP concentrations over $10 \%$ (Amable et al., 2014; Lucarelli et al., 2010), with opposing findings. Amable et al. (2014) demonstrated that PR-CM with PR concentrations over $10 \%$ had an inhibitory effect towards BM-MSC proliferation, whereas Lucarelli et al. (2010) demonstrated that PRF-CM at $20 \%$ induced stronger proliferation when compared to $10 \%$ PRF$\mathrm{CM}$. A dose-dependent increase in proliferation was also observed in a study comparing single and double PRF membranes (Dohan Ehrenfest et al., 2010).

The effects of leukocyte content in PP was only assessed by 3 studies, each using a different PP (Moisley et al., 2019; Perut et al., 2013; Yin et al., 2016). When compared against its leukocyte-poor counterparts, BM-MSCs incubated in leukocyterich PG-CM demonstrated greater proliferative capacity (Perut et al., 2013); whereas L-PRP-CM was found to be inferior (Yin et al., 2016). However, leukocyte content in PL was not found to influence proliferation (Moisley et al., 2019). Increasing platelet concentrations in PR was found to result in improved BM-MSC proliferation (Gruber et al., 2004).

The effect of incubation time on PPs was only assessed by 1 study (Parsons et al., 2008), whereby BM-MSCs incubated with $2.5 \%$ PRC showed more proliferation in the first $24 \mathrm{~h}$ only, with proliferation equal to that of the control group at $48 \mathrm{~h}$ and inferior to control group at $72 \mathrm{~h}$.

\section{Effects of PP on migration of BM-MSCs}

The chemotactic effects of PP on the migratory capacity of BM-MSCs was only assessed by 8 studies (Table 9). PRP-CM, PR, PRF-CM and PG$\mathrm{CM}$ demonstrated positive chemotactic effects on the migratory capacity of BM-MSC in vitro (Gruber et al., 2004; Nguyen et al., 2019; Schar et al., 2015; Yin et al., 2016). Most PL studies reported on its positive chemotactic effects (Infante et al., 2017; Moisley et al., 2019; Murphy et al., 2012). Migratory capacity of BMMSCs improved with increasing PP concentration (up to $10 \%$ ) in most studies that compared between different PP concentrations [PL: $n=2$ (Goedecke et al., 2011; Murphy et al., 2012) and PR: $n=2$ (Gruber et al., 2004; Nguyen et al., 2019)]. However, no studies have compared PP concentrations over $10 \%$. The effects of leukocyte content in PP on the migratory capacity of BM-MSCs was assessed by 2 studies, with different conclusions (Moisley et al., 2019; Yin et al., 2016). Whilst Yin et al. (2016) demonstrated that P-PRP-CM performed significantly better than L-PRP-CM, Moisley et al. (2019) demonstrated that leukocyte depletion do not affect BM-MSC migration.

\section{Effects of PPs on differentiation of BM-MSCs}

Table 10a-c summarises the effects of PPs on the differentiation potential of BM-MSCs into different mesenchymal lineages. Osteogenesis was most commonly assessed $(n=18)$, whilst adipogenesis 


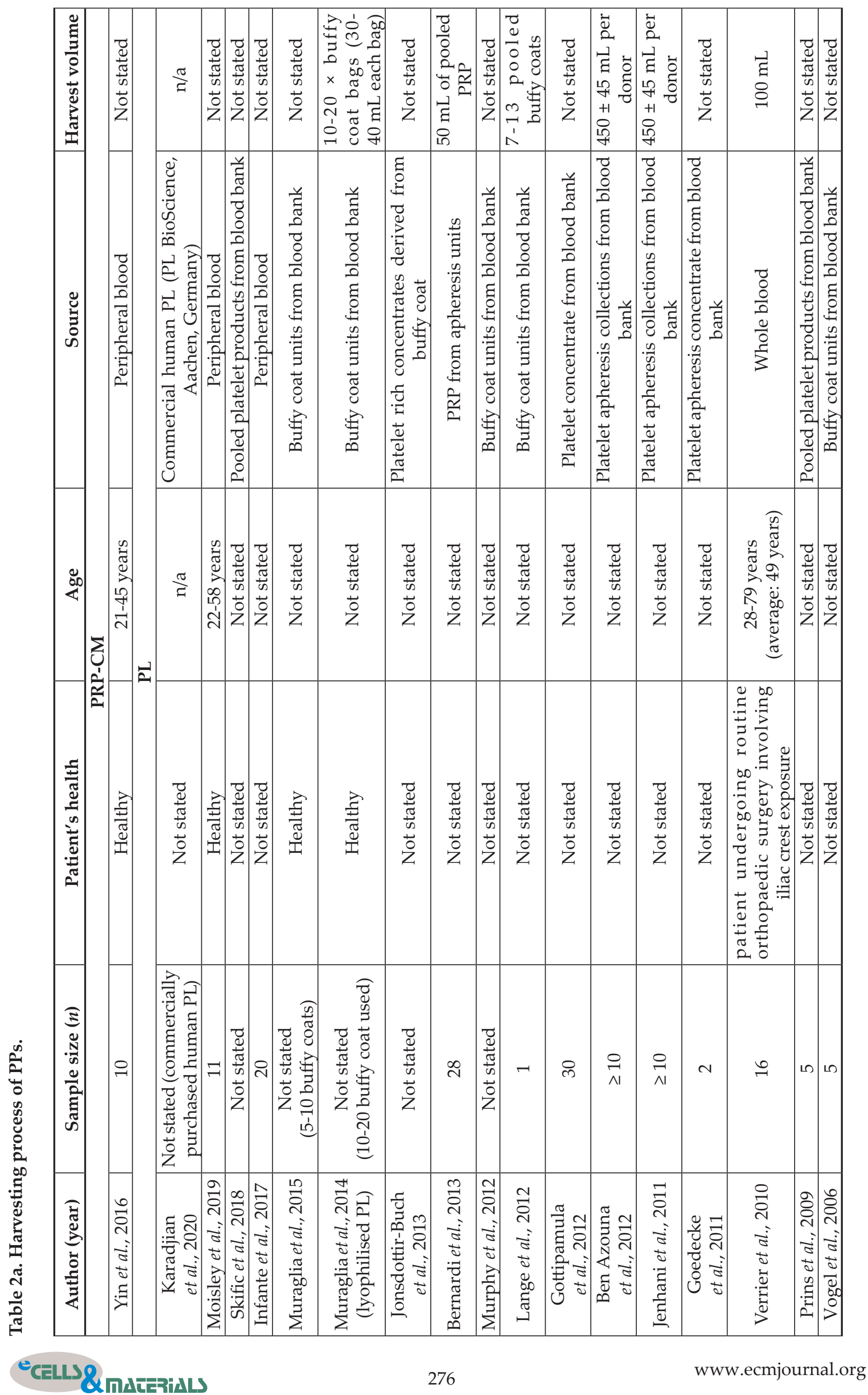




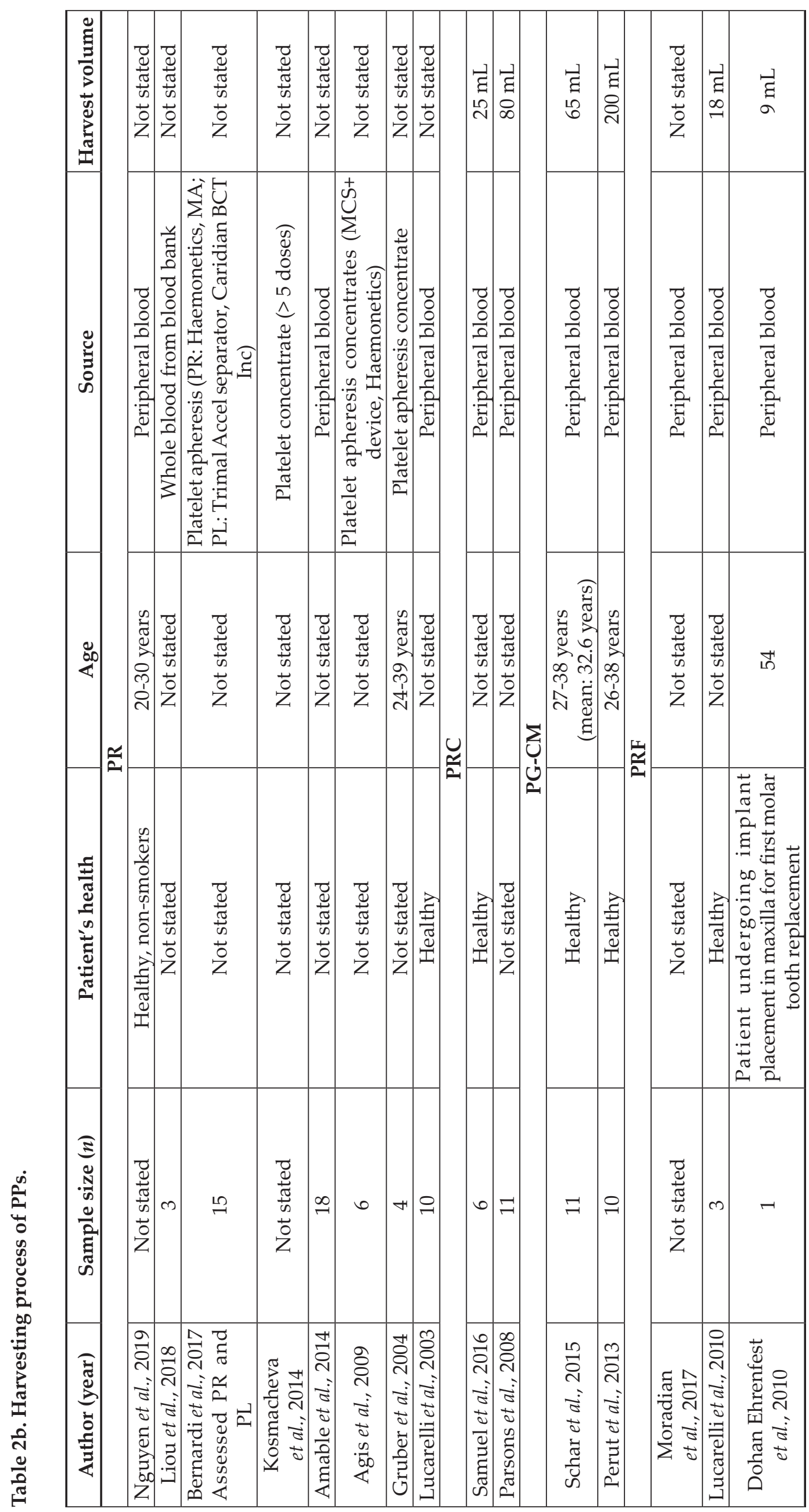




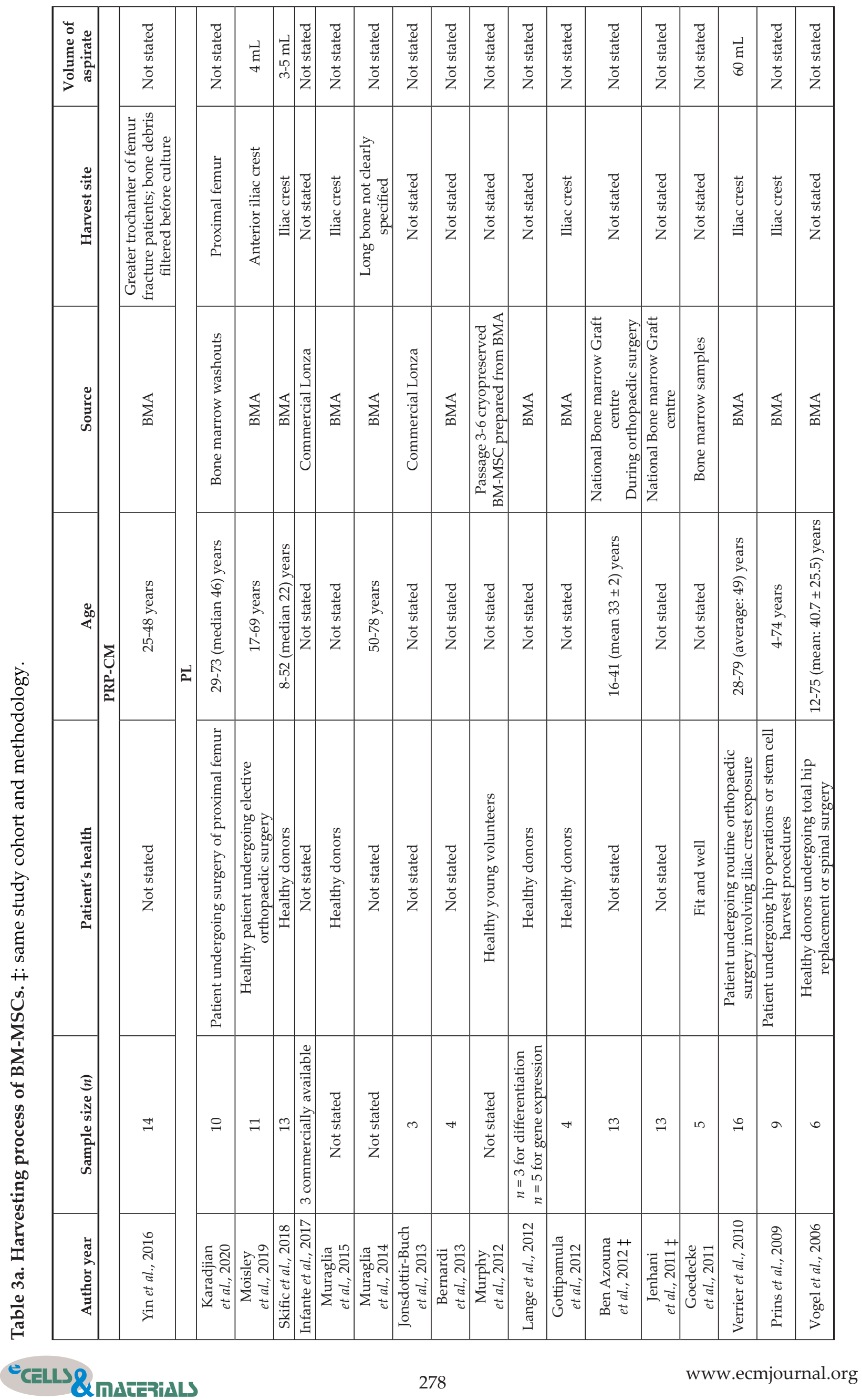




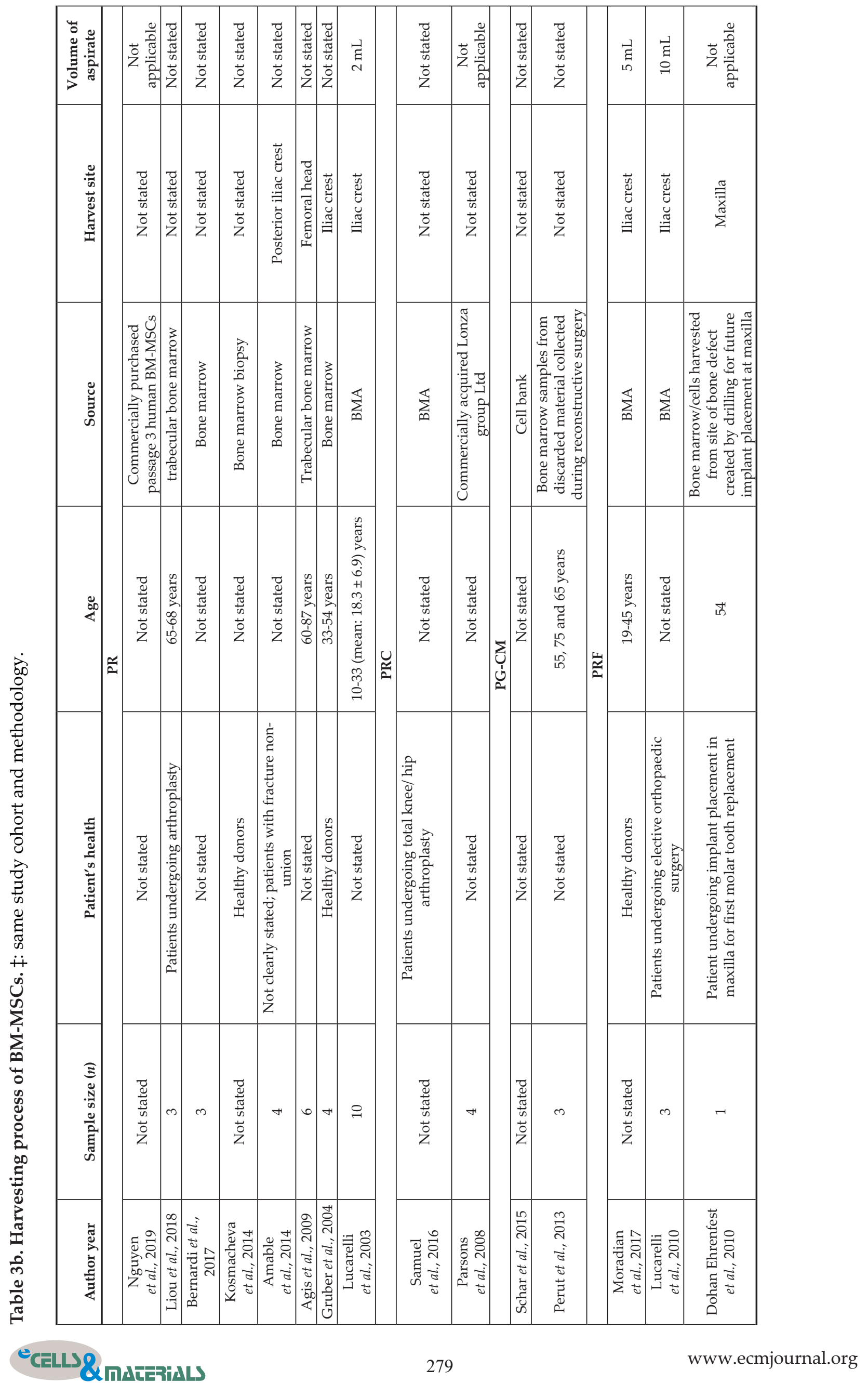


$(n=8)$ and chondrogenesis $(n=7)$ were less commonly investigated.

Osteogenic differentiation of BM-MSCs following PP exposure was found to be increased in most of the studies $(66.7 \%, n=12$ out of 18 studies) (Amable et al., 2014; Dohan Ehrenfest et al., 2010; Gottipamula et al., 2012; Karadjian et al., 2020; Kosmacheva et al., 2014; Lucarelli et al., 2003; Parsons et al., 2008; Perut et al., 2013; Samuel et al., 2016; Skific et al., 2018; Verrier et al., 2010; Yin et al., 2016). In contrast, PP exposure did not improve the adipogenic potential $(87.5 \%, n=7$ out of 8 studies) (Amable et al., 2014; Ben Azouna et al., 2012; Goedecke et al., 2011; Gottipamula et al., 2012; Lange et al., 2012; Prins et al., 2009; Vogel et al., 2006) and chondrogenic potential $(66.7 \%, n=4$ out of 6 studies) (Ben Azouna et al., 2012; Liou et al., 2018; Prins et al., 2009; Vogel et al., 2006) of BM-MSCs in the majority of the studies.

Only Lucarelli et al. (2003) investigated the effect of PP withdrawal on BM-MSC differentiation. They reported that despite PP withdrawal, BMMSCs previously exposed to PPs retained their improved osteogenic and chondrogenic capability, when compared against controls. Finally, only 2 studies investigated the effect of leukocyte content on osteogenic differentiation of BM-MSCs, with opposing findings (Perut et al., 2013; Yin et al., 2016).

\section{Effects of PPs on growth factor/cytokine/protein expression of BM-MSCs}

Table 11a,b summarises the findings of studies that have investigated the effect of PP on the expression of growth factors, cytokines, proteins and enzymes by BM-MSCs. Overall, PPs were found to have a chemotactic, pro-inflammatory, pro-osteogenic and pro-angiogenic effect on BM-MSCs. Chemotactic factors such as RANTES (Ben Azouna et al., 2012; Jenhani et al., 2011), SDF-1 $\alpha$ (Goedecke et al., 2011), eotaxin (Amable et al., 2014), IP-10 (Amable et al., 2014), MIP-1 $\beta$ (Amable et al., 2014) and MCP-1 (Amable et al., 2014) were upregulated in BM-MSCs exposed to PPs.

Pro-inflammatory cytokines (PGE2, NO, IL-2R, IL-6, IL-7, IL-8, IL-12 and IL-15) were universally upregulated by PPs in all in vitro studies (Amable et al., 2014; Ben Azouna et al., 2012; Jenhani et al., 2011; Yin et al., 2016). Comparing the effects of leukocyte content in PRP-CM on the release of pro-inflammatory cytokines PGE2 and NO by BM-MSCs, Yin et al. (2016) demonstrated that only L-PRP-CM upregulates the production of these pro-inflammatory cytokines significantly when compared to P-PRP-CM and controls; whereas no difference was observed when comparing P-PRP-CM to control (FBS).

Exposure to PPs was found to significantly upregulate most pro-osteogenic proteins by BMMSCs. These include osteocalcin (Samuel et al., 2016; Yin et al., 2016), RUNX2 (Yin et al., 2016) and BMP2 (Verrier et al., 2010). Comparing the effect of leukocyte concentration, Yin et al. (2016) reported stronger upregulation in P-PRP-CM in comparison to L-PRP-
CM $(p<0.001)$. With regards to angiogenesis, PR resulted in an increased release of thrombospondin (Amable et al., 2014), PLGF (Amable et al., 2014), aFGF (Amable et al., 2014), VEGF-D (Amable et al., 2014) by BM-MSCs (Amable et al., 2014). However, VEGF release was found to be equivocal [PR: upregulated $(n=1)$ (Amable et al., 2014); PL: less than control $(n=2)$ (Ben Azouna et al., 2012; Jenhani et al., 2011)]. On the other hand, the release of adipogenic molecules L-PGDS (Lange et al., 2012) and leptin (Ben Azouna et al., 2012) by BM-MSCs following PP exposure was found to be weaker than control group.

PPs increased the secretion of catabolic MMPs (MMP1, MMP3, MMP7, MMP8 and MMP13) and extracellular matrix proteins and other molecules (heparan sulphate, elastin, laminin, collagens I and III) (Amable et al., 2014). Amable et al. (2014) studied also the release of other growth factors and found that PP increases the secretion of HGF, G-CSF, TGF- $\beta 1$ and TGF- $\beta 2$; whereas it downregulates PDGF-BB secretion.

\section{Effects of PPs on other BM-MSC functions}

The effect of PPs on the immunomodulatory function of BM-MSCs was assessed by 4 studies (Bernardi et al., 2017; Gottipamula et al., 2012; Jonsdottir-Buch et al., 2013; Yin et al., 2016) (Table 12). Whilst L-PRP$\mathrm{CM}$ was found to induce the activation of the NF$\kappa \mathrm{B}$ signalling pathway, which could activate genes responsible for inflammation and other immune responses (Yin et al., 2016), the immunomodulatory effects of BM-MSCs exposed to PL seemed to be equivocal. Jonsdottir-Buch et al. (2013) demonstrated PL to have no effect on the ability of BM-MSCs to reduce mononuclear cell proliferation; whilst 2 other studies demonstrated BM-MSCs cultured with PL to have weaker inhibitory effects on PBMCs (Bernardi et al., 2017) and T-cell proliferation (Gottipamula et al., 2012) when compared to controls.

Other biophysiological function of BM-MSCs assessed were (i) response towards apoptotic stress, (ii) chemotactic effect on HSCs and (iii) capacity to stimulate plasminogen activation. Yin et al. (2016) found that both L-PRP- and P-PRP-CM are able to inhibit camptothecin-induced apoptosis of BM-MSCs and, therefore, enhance its viability, with L-PRP$\mathrm{CM}$ having the strongest effect. The chemotactic effect of BM-MSCs in terms of inducing migration of HSCs was only assessed by 1 study, whereby the chemotactic effect of BM-MSCs cultured in $5 \%$ and $10 \%$ PL was demonstrably inferior when compared to control (Goedecke et al., 2011). Agis et al. (2009) found that BM-MSCs pre-incubated with PR demonstrated a stronger capacity to stimulate plasminogen activation and fibrinolysis.

\section{Gene expression of BM-MSCs following PP exposure}

Table 13a-c summarises the effect PP has on gene expression of BM-MSCs, based on the biophysiological functions of BM-MSCs. Samples were taken from BM- 


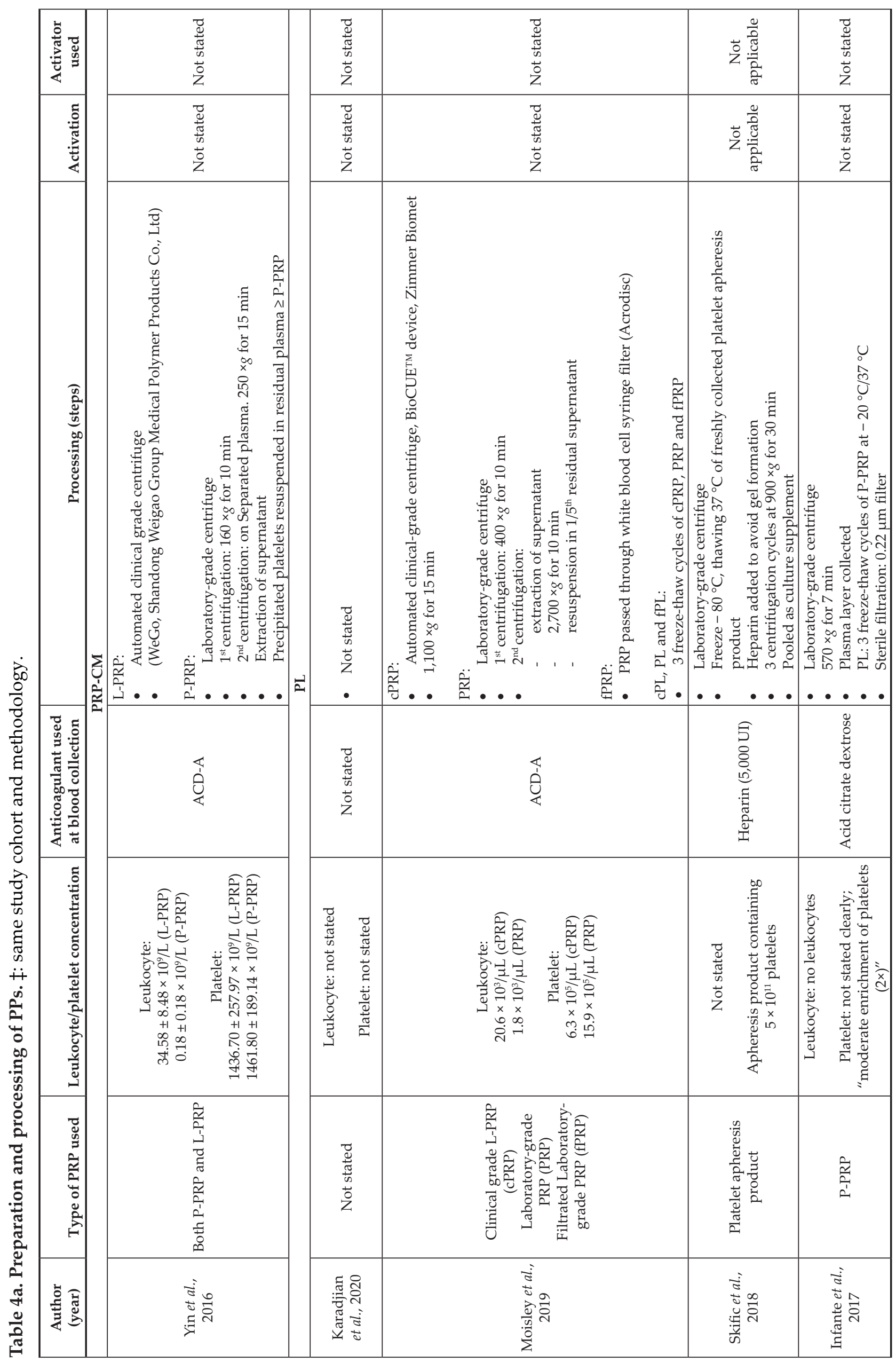




\begin{tabular}{|c|c|c|c|c|c|c|c|c|c|}
\hline 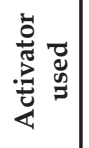 & 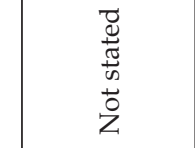 & 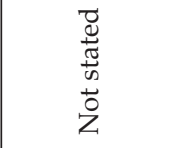 & 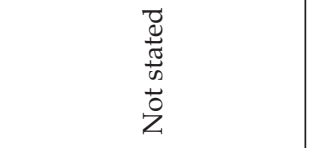 & 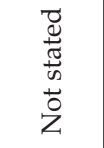 & 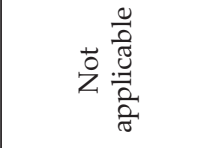 & 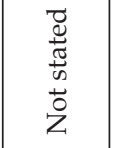 & 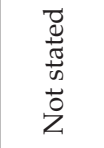 & 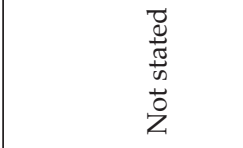 & \\
\hline 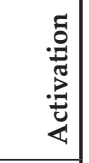 & 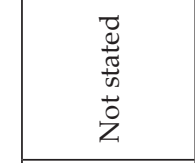 & 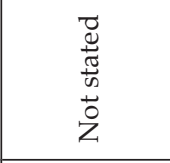 & 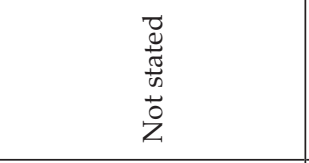 & 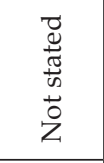 & Z & 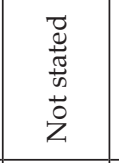 & $\begin{array}{l}\vec{J} \\
\vec{d} \\
\frac{\pi}{5} \\
0 \\
0 \\
z\end{array}$ & $\begin{array}{l}\overline{0} \\
\frac{\pi}{\pi} \\
0 \\
0 \\
0 \\
Z\end{array}$ & \\
\hline 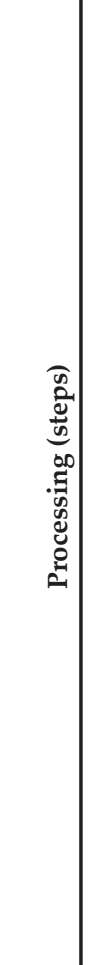 & 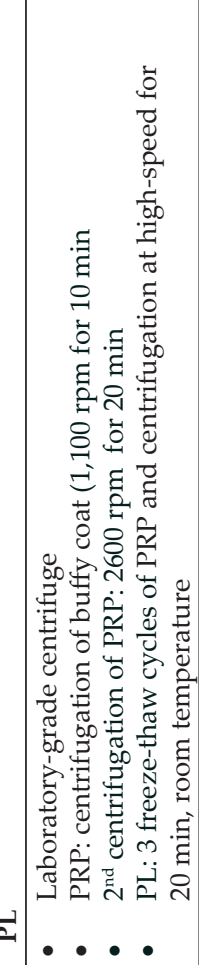 & 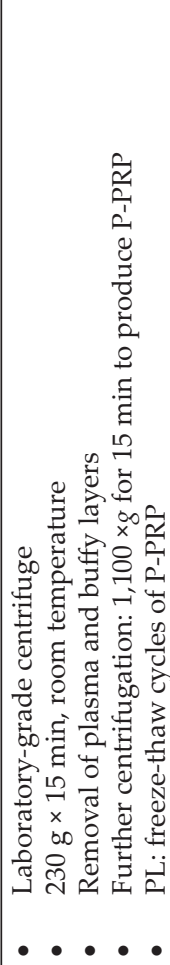 & 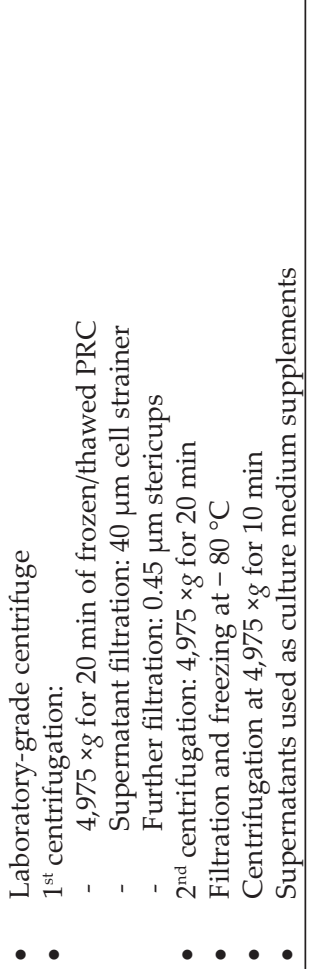 & 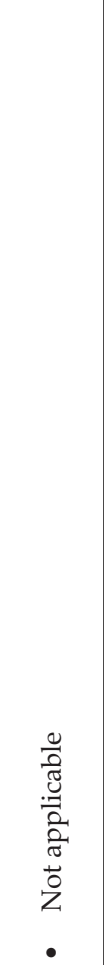 & 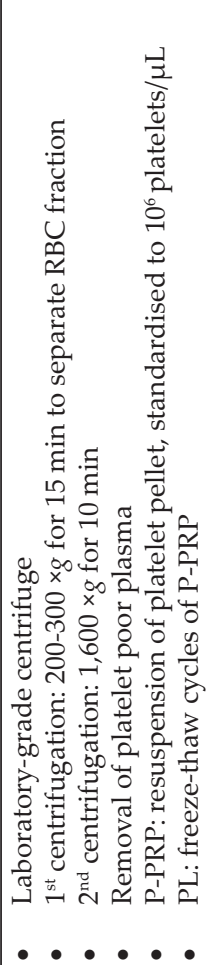 & 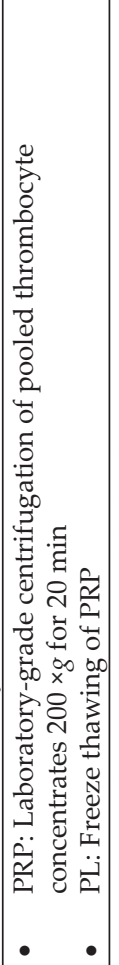 & 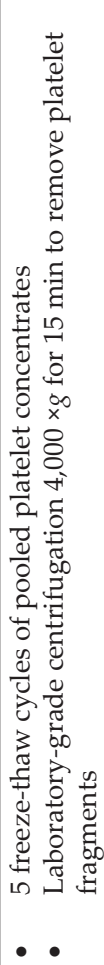 & 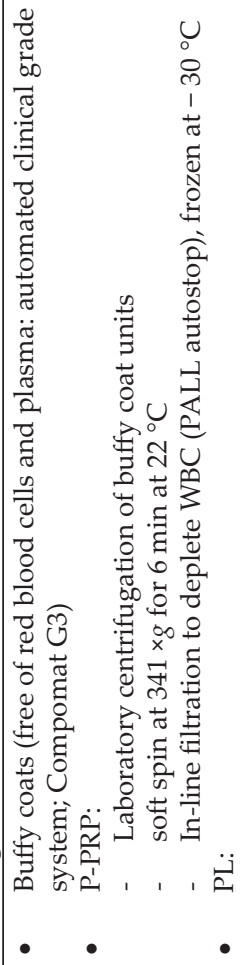 & 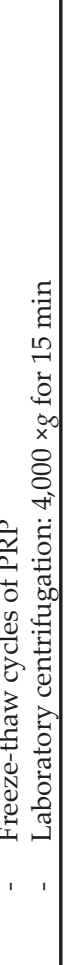 \\
\hline 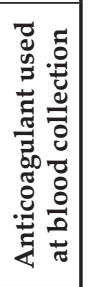 & 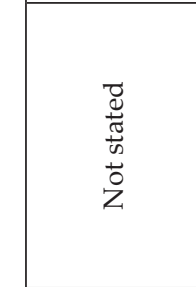 & 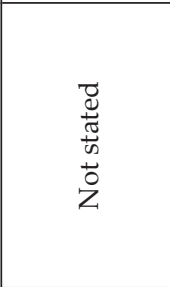 & 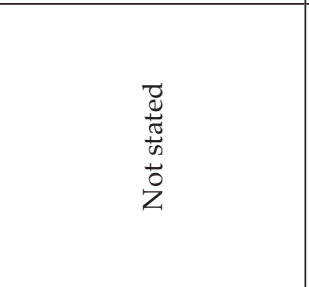 & 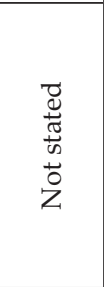 & 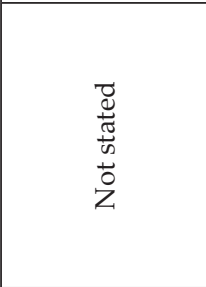 & 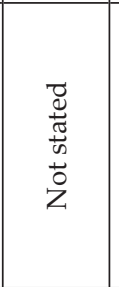 & 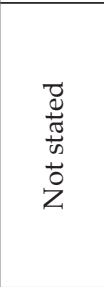 & 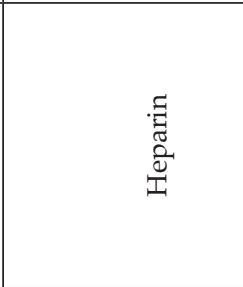 & \\
\hline 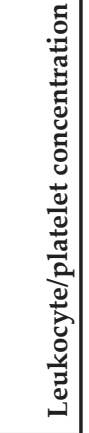 & 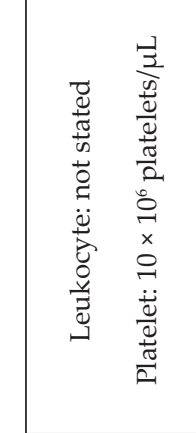 & 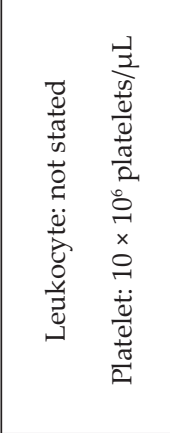 & 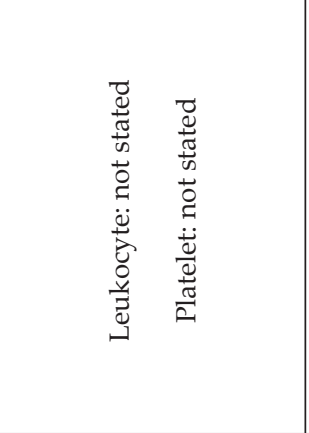 & 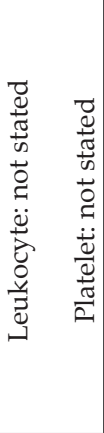 & 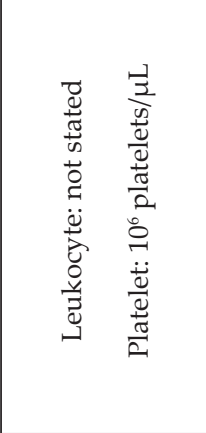 & 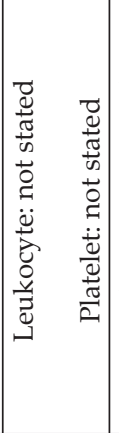 & 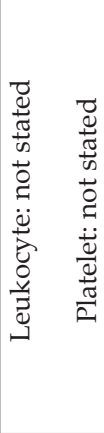 & 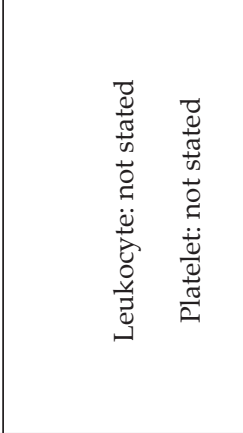 & \\
\hline 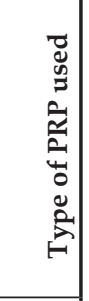 & 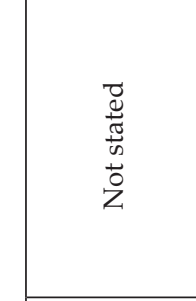 & 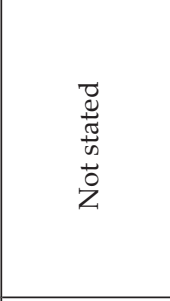 & 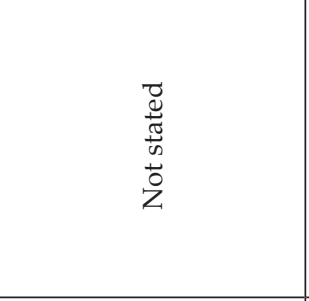 & 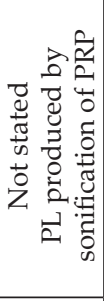 & 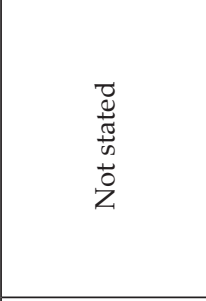 & 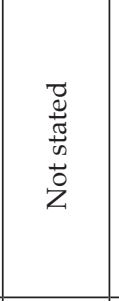 & 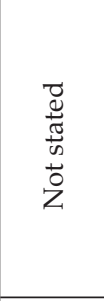 & 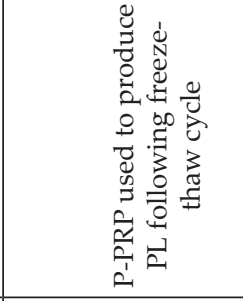 & \\
\hline 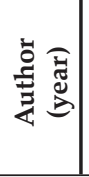 & 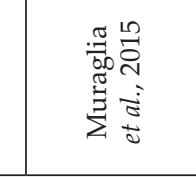 & 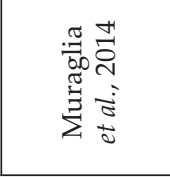 & 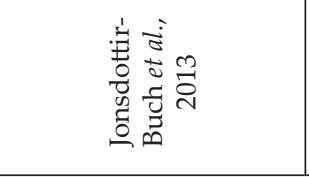 & 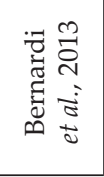 & 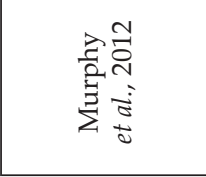 & 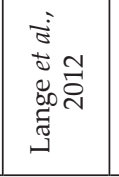 & 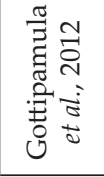 & 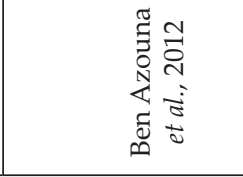 & \\
\hline
\end{tabular}




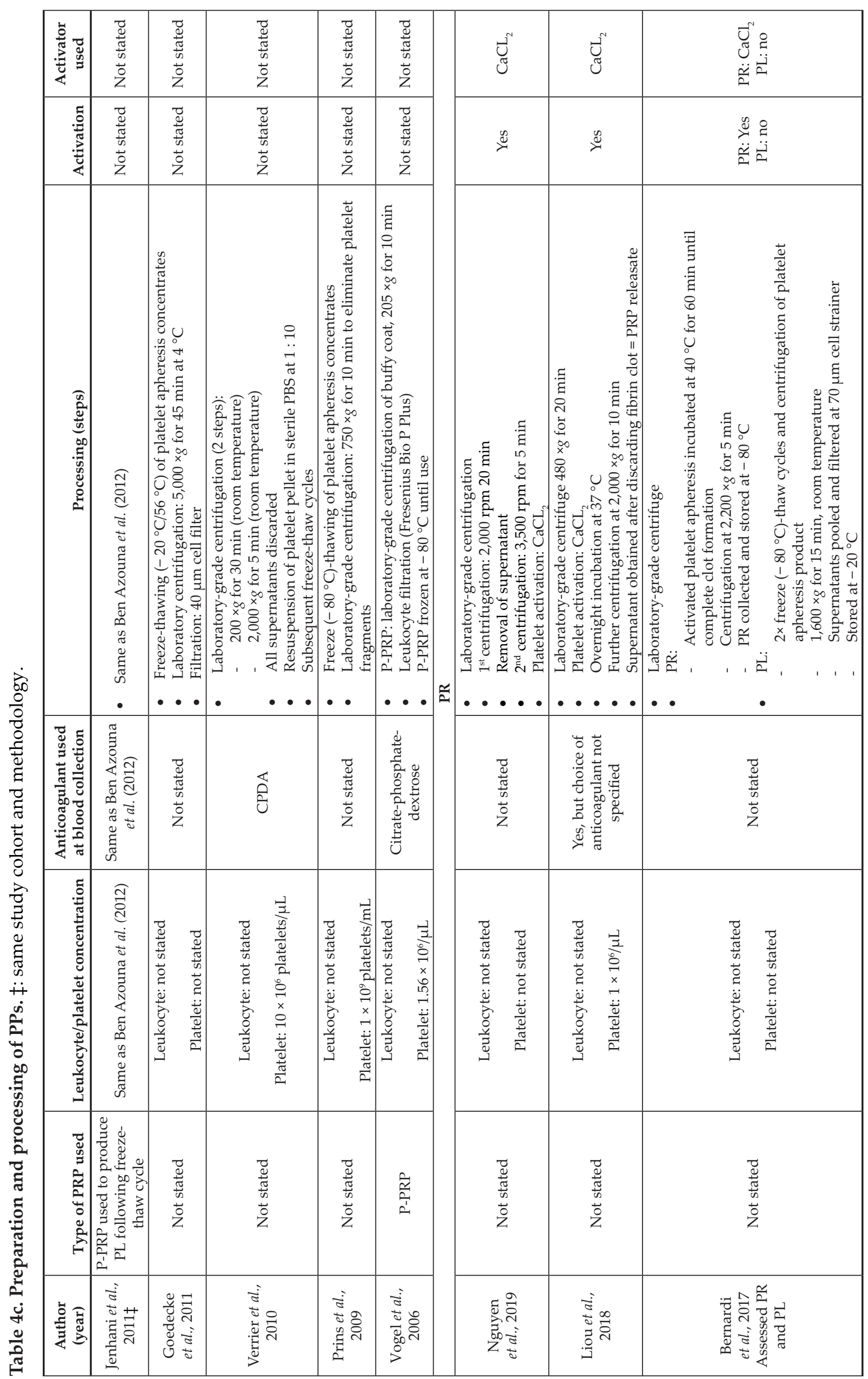




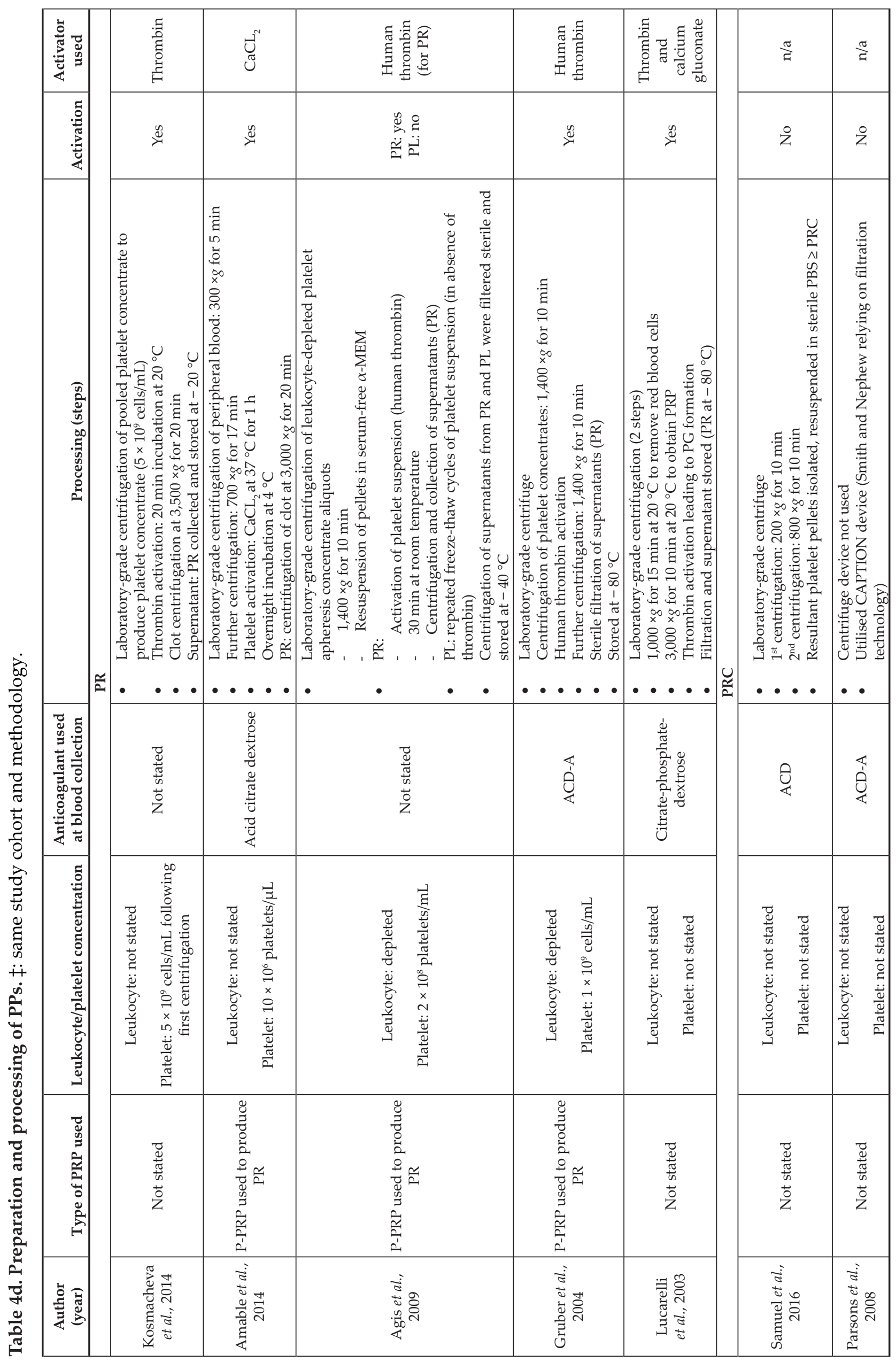




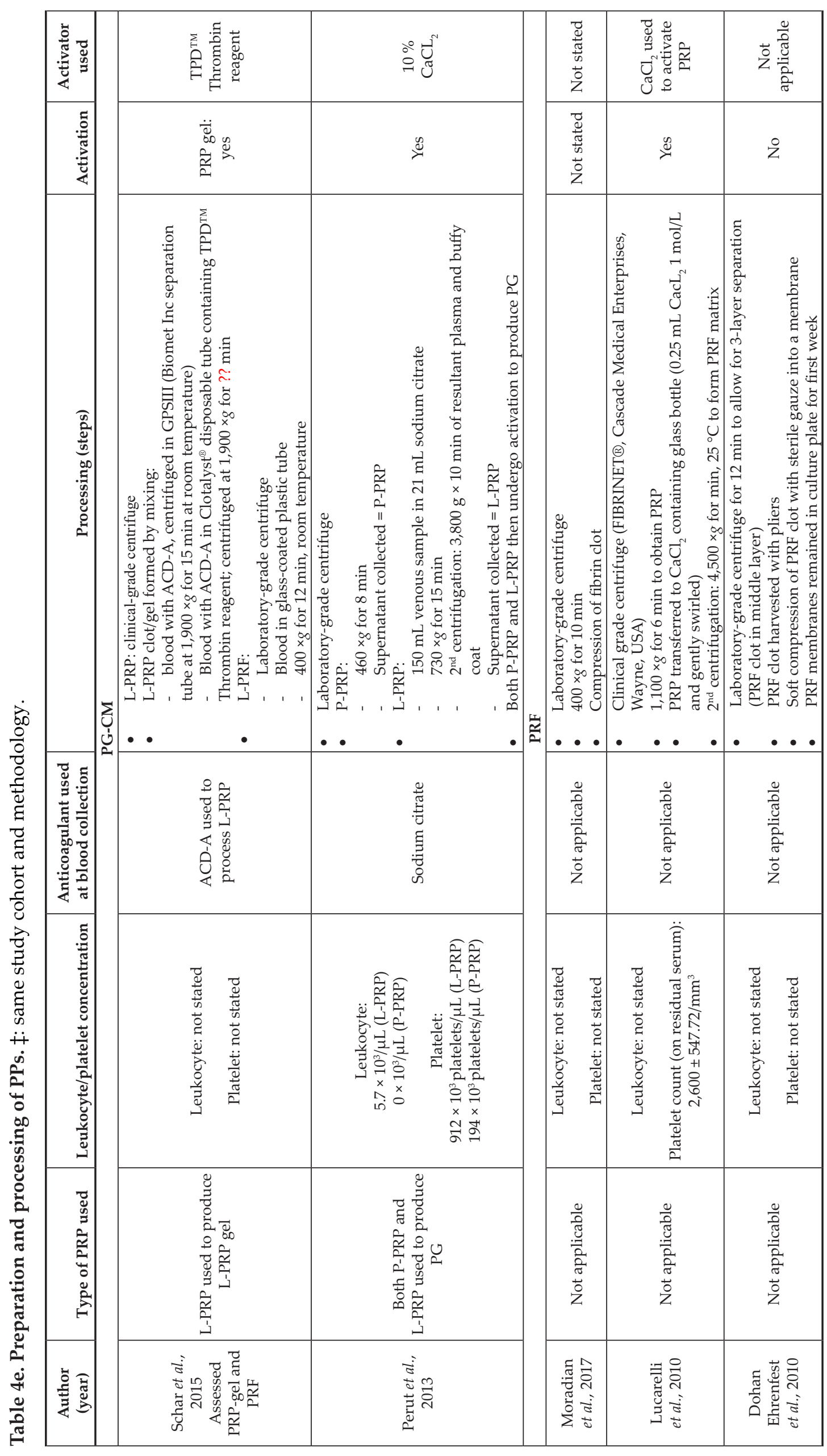




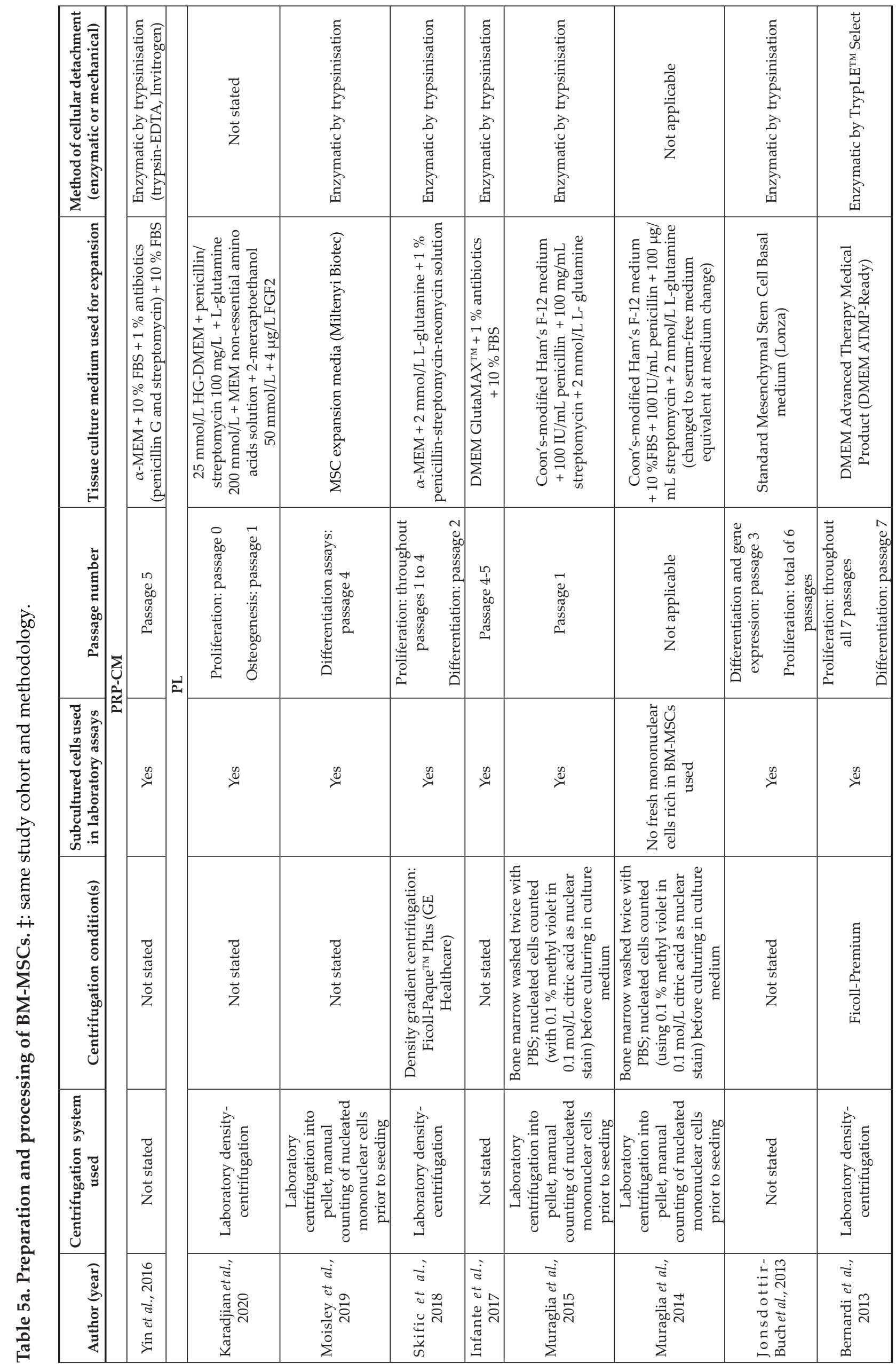




\begin{tabular}{|c|c|c|c|c|c|c|c|c|c|c|c|}
\hline 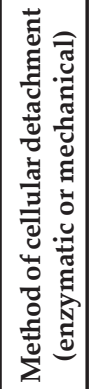 & 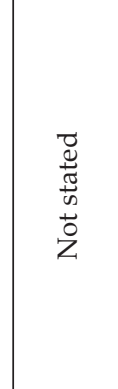 & 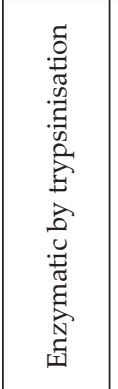 & 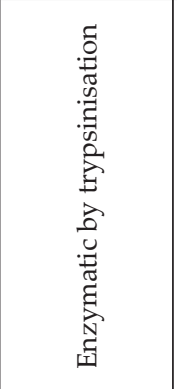 & 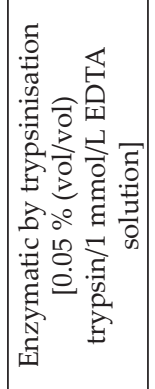 & 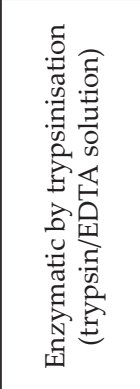 & 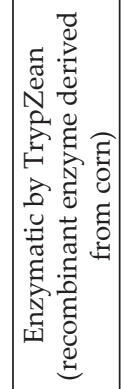 & 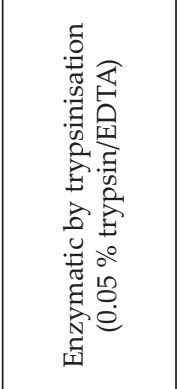 & 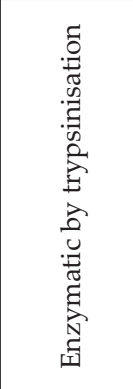 & 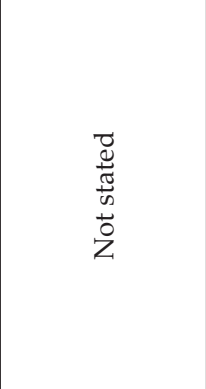 & 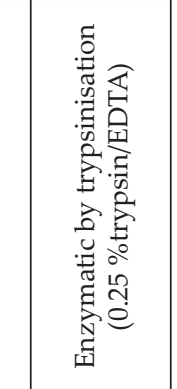 & 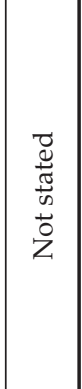 \\
\hline 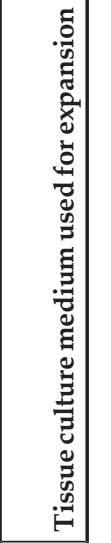 & 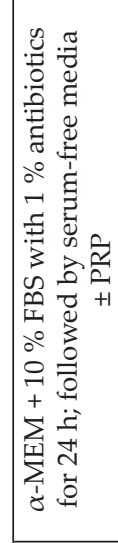 & 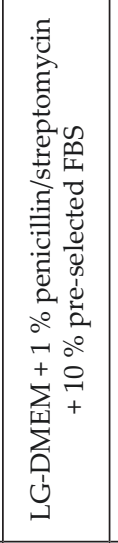 & 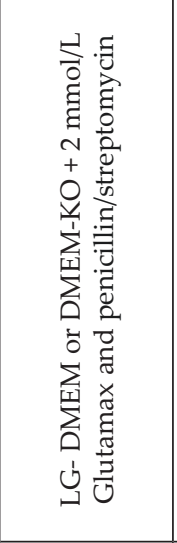 & 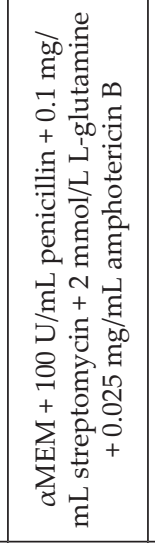 & 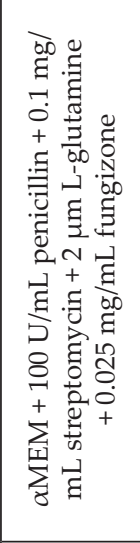 & 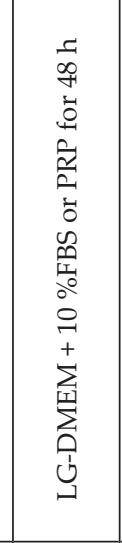 & 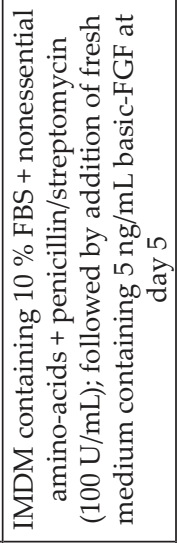 & 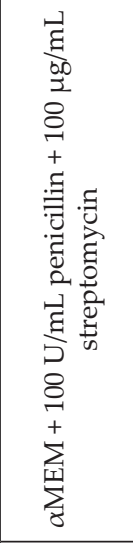 & 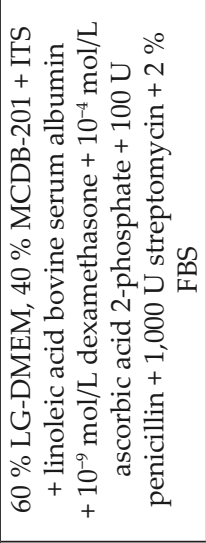 & 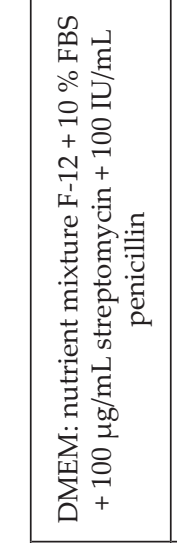 & 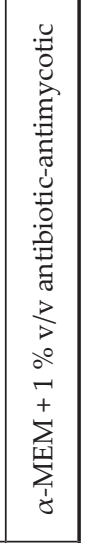 \\
\hline 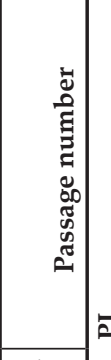 & 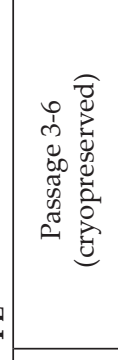 & 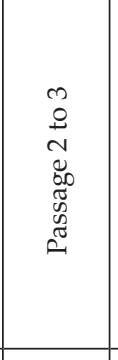 & 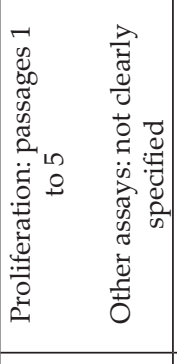 & 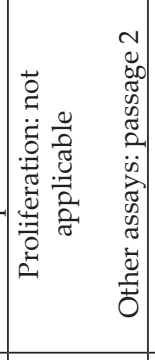 & 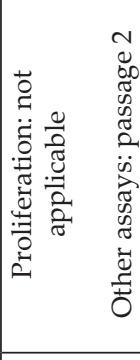 & 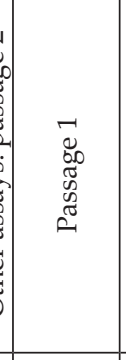 & 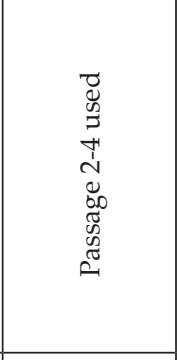 & 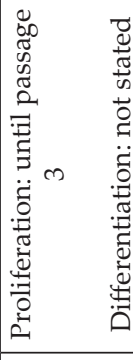 & 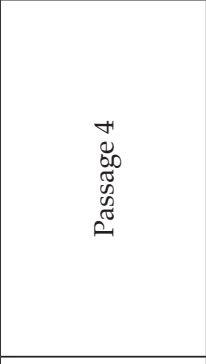 & 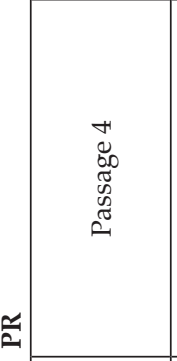 & 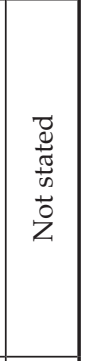 \\
\hline 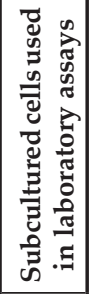 & ্्ֻर & ఫ્र & 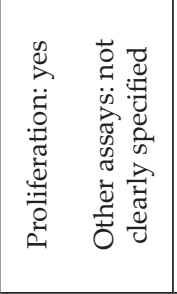 & 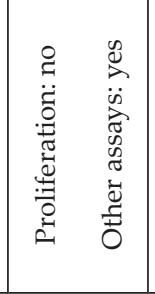 & 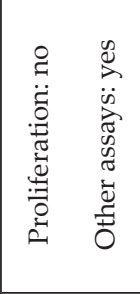 & $\stackrel{\mathscr{D}}{2}$ & $\overbrace{્}^{\mathscr{V}}$ & ્्ֻर & $\stackrel{\mathscr{C}}{\varnothing}$ & $\stackrel{D}{0}$ & $\stackrel{0}{\varnothing}$ \\
\hline 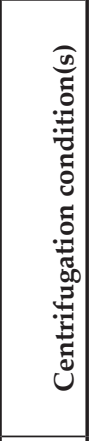 & $\begin{array}{l}\bar{D} \\
\frac{\pi}{\pi} \\
\frac{1}{\omega} \\
0 \\
z\end{array}$ & 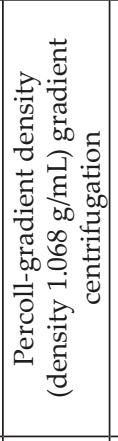 & 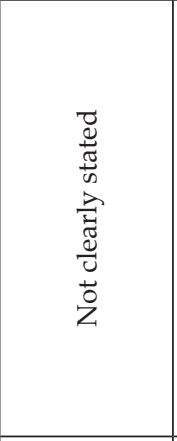 & 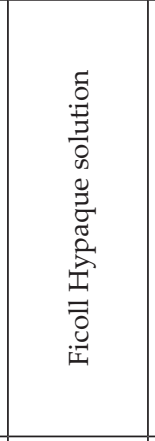 & 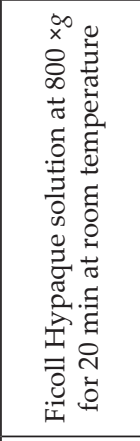 & 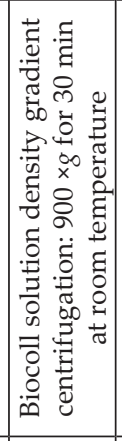 & 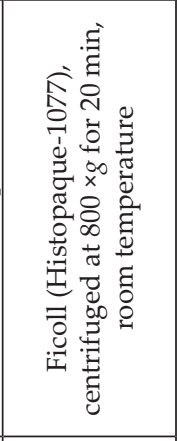 & 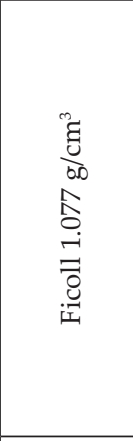 & 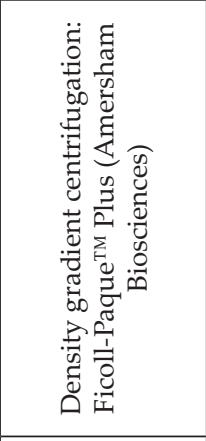 & 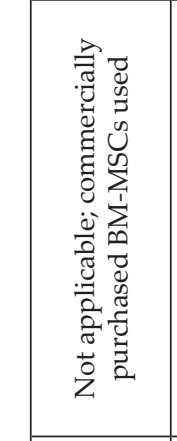 & 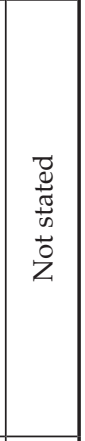 \\
\hline 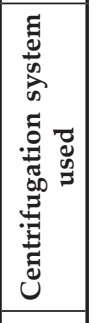 & $\begin{array}{l}\overline{0} \\
\frac{\pi}{\pi} \\
\frac{1}{0} \\
0 \\
z\end{array}$ & 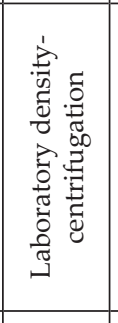 & 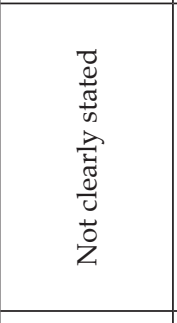 & 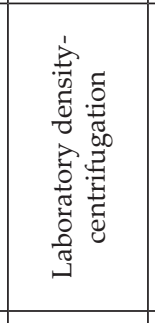 & 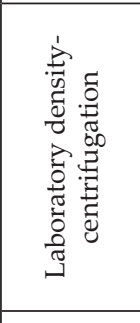 & 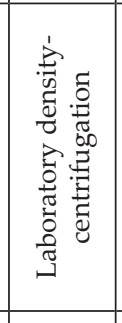 & 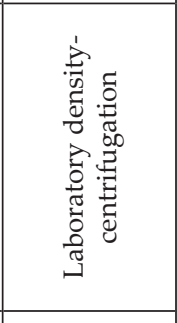 & 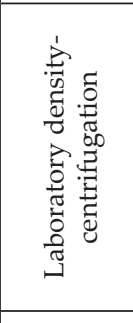 & 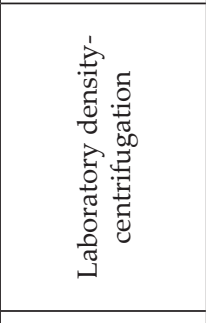 & 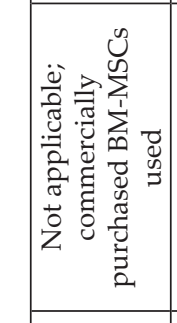 & 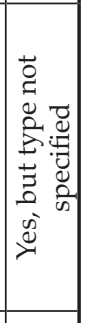 \\
\hline 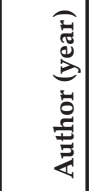 & 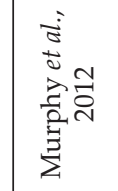 & 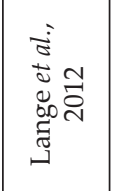 & 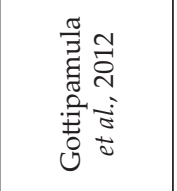 & 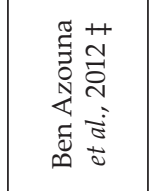 & 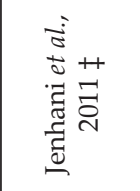 & 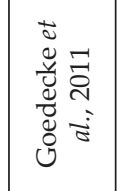 & 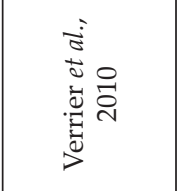 & 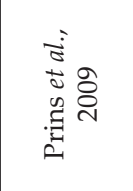 & 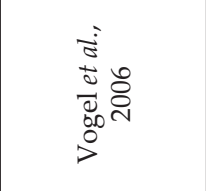 & 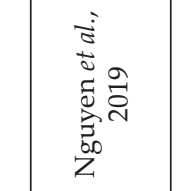 & 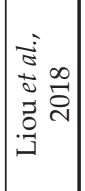 \\
\hline
\end{tabular}




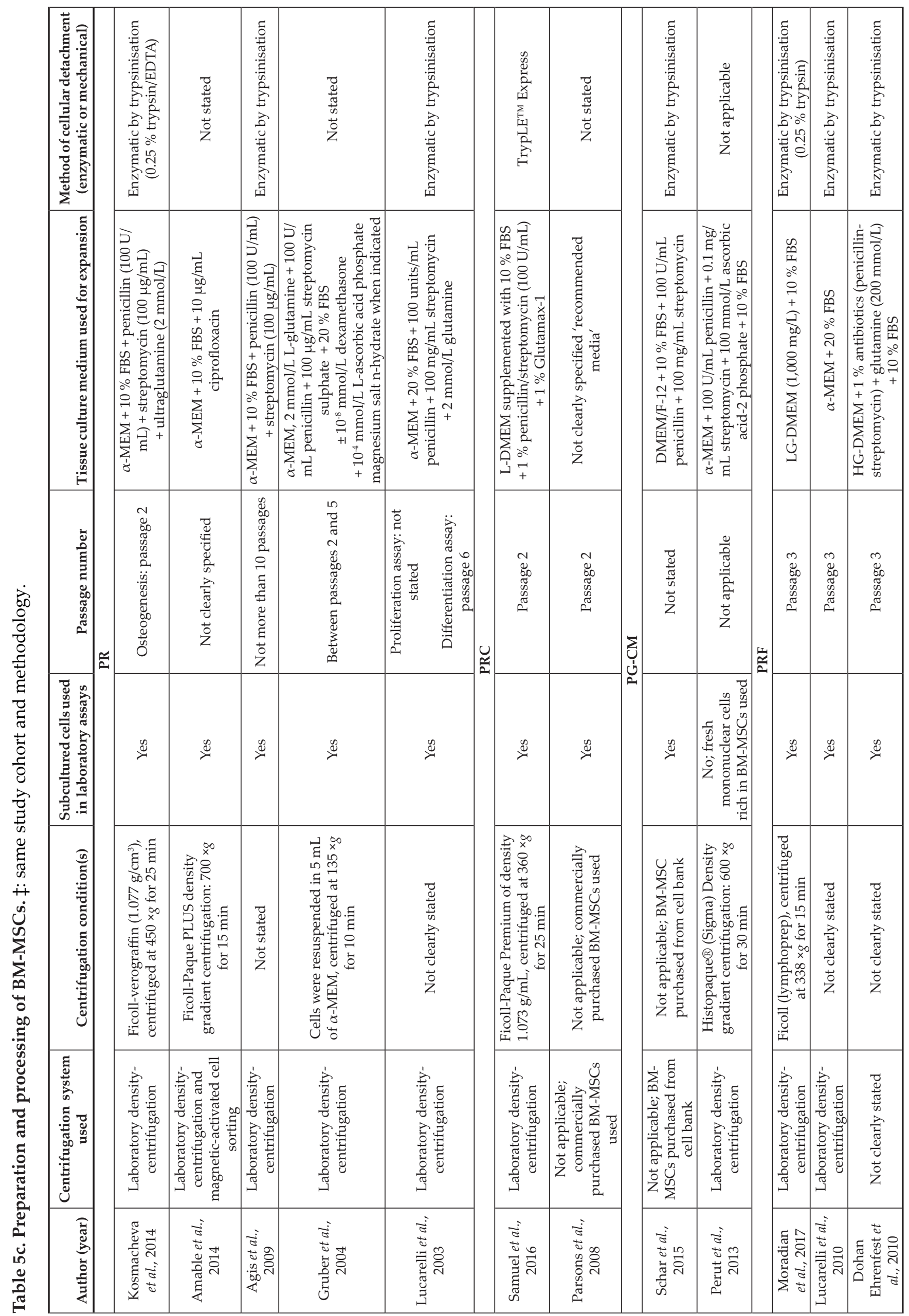




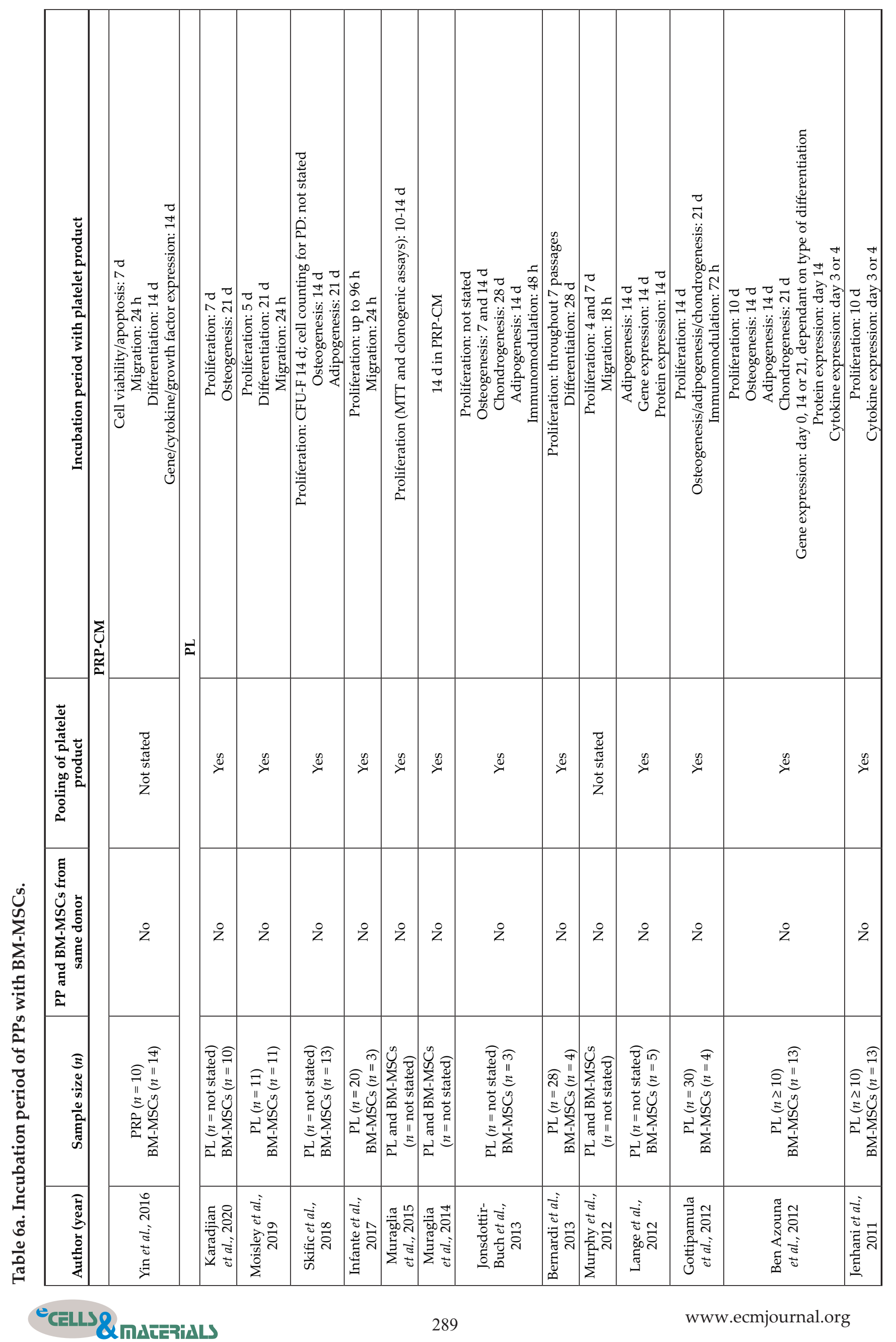




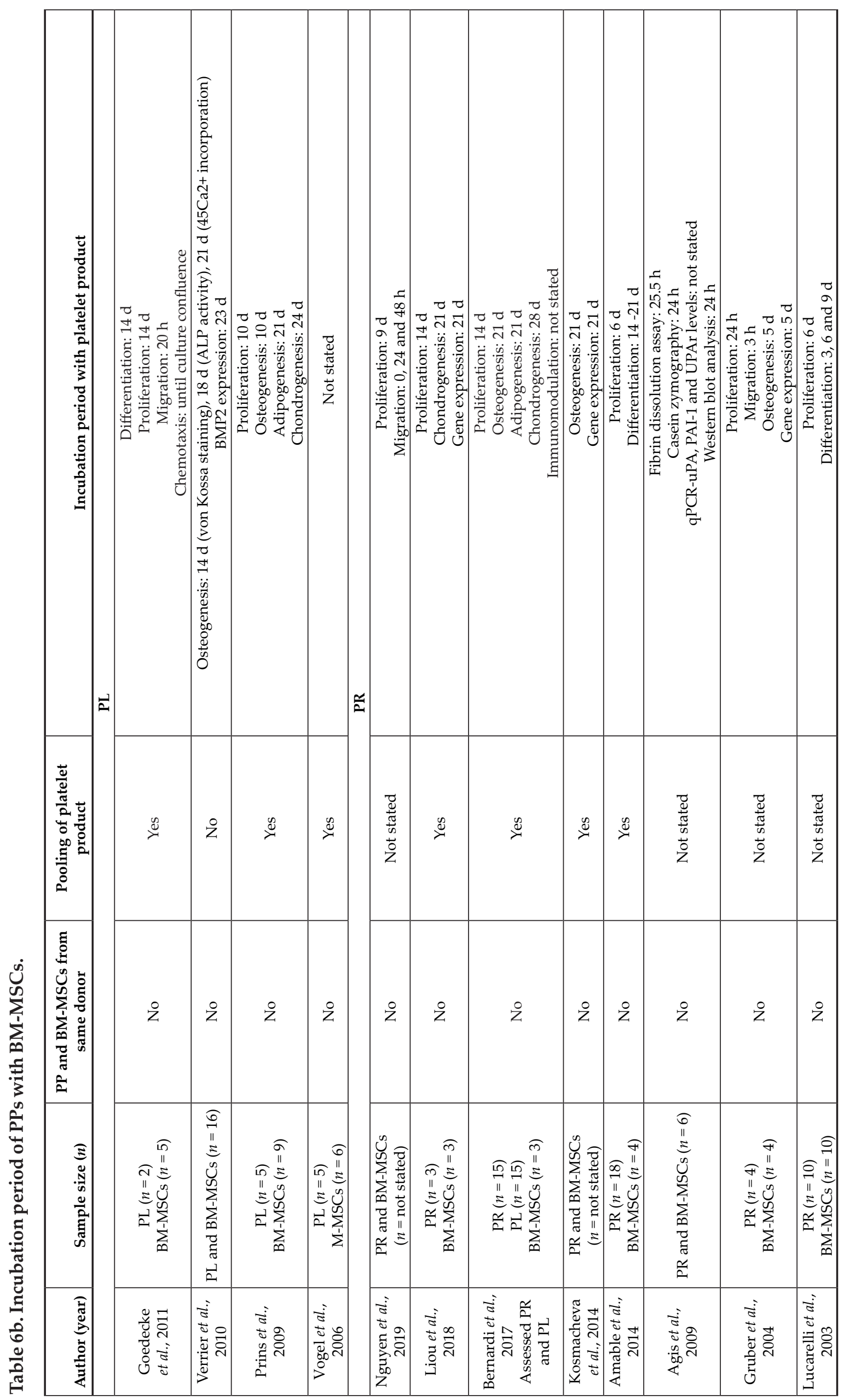




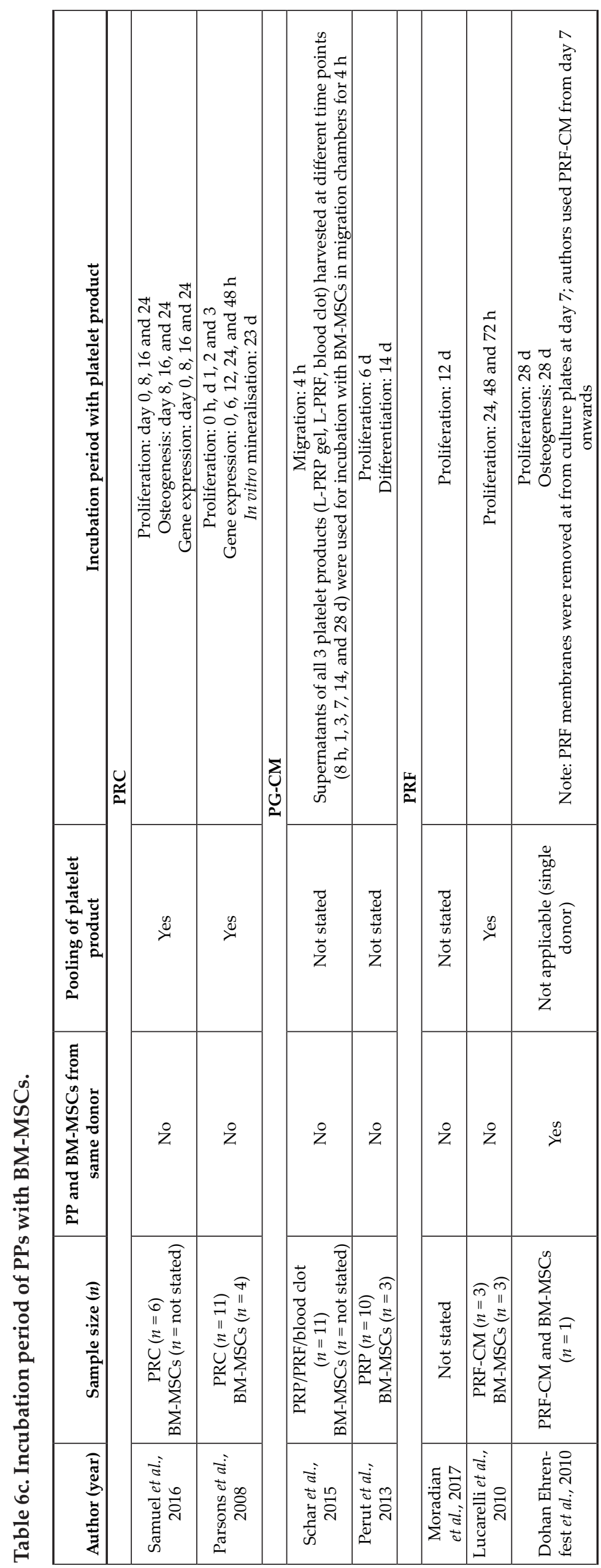


MSCs incubated in PRP-CM $(n=1)$, PL $(n=6)$ and $\operatorname{PRC}(n=2)$.

$\mathrm{PP}$ was found to upregulate the expression of BM-MSC gene markers for pluripotency and proliferation (POU5F1, SOX2) (Amable et al., 2014); immunomodulation/inflammation (COX-2, iNOS) (Yin et al., 2016) and matrix metalloproteinases (MMP13) (Verrier et al., 2010).

The effect of PP on expression of osteogenic gene markers was variable, dependent upon the gene marker measured and choice of PP used. Osteogenic genes such as BMP2 (Amable et al., 2014; Samuel et al., 2016; Verrier et al., 2010), osteocalcin (Gottipamula et al., 2012; Yin et al., 2016), osteopontin (Jonsdottir-Buch et al., 2013; Kosmacheva et al., 2014; Samuel et al., 2016), bone sialoprotein 2 (Samuel et al., 2016; Verrier et al., 2010), osterix (Gottipamula et al., 2012) and COL1 (Samuel et al., 2016) were upregulated in most studies. On the other hand, PP exposure resulted in equivocal expression of ALP (Jonsdottir-Buch et al., 2013; Parsons et al., 2008; Samuel et al., 2016; Verrier et al., 2010) and RUNX2 (Amable et al., 2014; Gruber et al., 2004; Jonsdottir-Buch et al., 2013; Parsons et al., 2008; Samuel et al., 2016; Yin et al., 2016) as well as downregulation of SPARC (Amable et al., 2014) and osteonectin (Samuel et al., 2016).

Effects of PP on expression of adipogenic gene markers of BM-MSCs were variable: $A D I P O Q$ (Amable et al., 2014), CEPBA (Amable et al., 2014) and PPARG (Amable et al., 2014) were upregulated; whilst L-PGDS (Lange et al., 2012), LPL (Ben Azouna et al., 2012), FABP4 (Lange et al., 2012), PLIN (Lange et al., 2012), GLUT4 (Lange et al., 2012) and APOE (Lange et al., 2012) were downregulated when compared to controls. Similarly, expression of chondrogenic gene markers were variable: COMP (Infante et al., 2017) and COL1A1 (Verrier et al., 2010) were upregulated; COL2A1 downregulated (Infante et al., 2017); ACAN (Amable et al., 2014; Infante et al., 2017; Liou et al., 2018) and SOX9 (Amable et al., 2014; Infante et al., 2017) equivocal. No effect on COL2 was observed (Liou et al., 2018).

\section{Discussion}

PPs and BM-MSCs are increasingly used in the current treatment of impaired bone healing. However, the clinical effectiveness of their combined use is difficult to conclude, due to differing clinical results and the huge heterogeneity in terms of harvesting, preparation techniques, contents and type of PPs used in these studies. Pre-clinical studies using animal models have their limitations, since animal cells do not always resemble human results. Systematically assessing the results of in vitro studies in humans should provide the best collective evidence which can guide clinical practice. This review represents the first systematic review in the literature that specifically assessed the effects of PPs on the biophysiological functions of BM-MSCs in human.
This systematic review has identified 33 in vitro studies in humans that compared the combined use of PP and BM-MSCs to BM-MSCs alone. It was evident that there is a lack of consistent terminology amongst these studies. For instance, several studies used the term PRP, which upon closer look at their methodology, were in fact using frozen-thawed PRP (Goedecke et al., 2011; Infante et al., 2017; Muraglia et al., 2014; Murphy et al., 2012; Vogel et al., 2006). The freeze-thawing process of the PRP in these studies would have resulted in lysis of platelets. Thus, PL would have been a more appropriate term to accurately reflect the acellularity and contents of the PP used in these studies. Therefore, the present study attempted to clearly categorise these studies into PRP-CM, PL, PR, PRC, PG-CM and PRF to reflect (i) the specific type of PP used, (ii) its consistency (i.e. liquid, solid) and (iii) its contents (e.g. cellularity, lysed platelets, leukocyte). Hopefully, this would aid comparison between the important findings of these in vitro studies with future laboratory work and aid in its transferability into clinical work.

The majority of these studies had a small sample size $(n \leq 6)$ (Agis et al., 2009; Amable et al., 2014; Bernardi et al., 2017; Bernardi et al., 2013; Dohan Ehrenfest et al., 2010; Goedecke et al., 2011; Gottipamula et al., 2012; Gruber et al., 2004; Infante et al., 2017; Liou et al., 2018; Lucarelli et al., 2010; Parsons et al., 2008; Perut et al., 2013; Prins et al., 2009; Vogel et al., 2006). Key aspects such as the health and demographics of patients/volunteers, harvest volume of BM-MSCs and PPs as well as the leukocyte and platelet concentration in PP were not reported by most studies, which would have otherwise substantiated the correct interpretation of the results reported. Harvested samples were processed using different centrifugation systems, mostly being laboratory grade (Agis et al., 2009; Amable et al., 2014; Ben Azouna et al., 2012; Bernardi et al., 2017; Dohan Ehrenfest et al., 2010; Goedecke et al., 2011; Gottipamula et al., 2012; Gruber et al., 2004; Infante et al., 2017; Jenhani et al., 2011; Jonsdottir-Buch et al., 2013; Kosmacheva et al., 2014; Lange et al., 2012; Liou et al., 2018; Muraglia et al., 2014; Muraglia et al., 2015; Murphy et al., 2012; Perut et al., 2013; Prins et al., 2009; Samuel et al., 2016; Schar et al., 2015; Skific et al., 2018; Verrier et al., 2010; Vogel et al., 2006). Comparisons between PL produced using a laboratory and clinical grade centrifugation system on proliferation and migration of BM-MSCs have been attempted (Moisley et al., 2019), but not yet on the other categories of PPs. Furthermore, the practice of pooling harvested PPs was common practice within the majority of in vitro studies analysed (Amable et al., 2014; Ben Azouna et al., 2012; Bernardi et al., 2017; Bernardi et al., 2013; Goedecke et al., 2011; Gottipamula et al., 2012; Infante et al., 2017; Jenhani et al., 2011; Jonsdottir-Buch et al., 2013; Karadjian et al., 2020; Kosmacheva et al., 2014; Lange et al., 2012; Liou et al., 2018; Lucarelli et al., 2010; Moisley et al., 2019; Muraglia et al., 2014; Muraglia et al., 2015; Parsons et al., 2008; Prins et al., 2009; Samuel 
et al., 2016; Skific et al., 2018; Vogel et al., 2006). This is not reflective of clinical practice, where patients are commonly treated with autologous PP and BM-MSCs (i.e. donor-matched) processed using a clinical-grade automated centrifugation system (Lee et al., 2014; Martin et al., 2013).

Being a biological reservoir containing a panel of potent biological factors, it is unsurprising that most in vitro studies have been focused upon harnessing these platelet secretomes (e.g. cytokines, growth factors, chemokines) either through physical lysis (e.g. freeze-thawing) or through exogenous chemical activation (e.g. $\mathrm{CaCl}_{2}$, thrombin). Noteworthy, these methods in essence lead to the immediate secretion of the potent platelet secretomes upon delivery (Marx, 2001; 2004). Therefore, it is unsurprising that most in vitro studies utilised PL (Agis et al., 2009; Ben Azouna et al., 2012; Bernardi et al., 2017; Bernardi et al., 2013; Goedecke et al., 2011; Gottipamula et al., 2012; Gruber et al., 2004; Infante et al., 2017; Jenhani et al., 2011; Jonsdottir-Buch et al., 2013; Kosmacheva et al., 2014; Lange et al., 2012; Moisley et al., 2019; Muraglia et al., 2014; Muraglia et al., 2015; Murphy et al., 2012; Prins et al., 2009; Skific et al., 2018; Vogel et al., 2006), PR (Agis et al., 2009; Amable et al., 2014; Bernardi et al., 2017; Gruber et al., 2004; Kosmacheva et al., 2014; Liou et al., 2018; Lucarelli et al., 2003; Nguyen et al., 2019) and PG-CM (Perut et al., 2013; Schar et al., 2015), all of which were induced to release platelet secretomes through its manufacturing process. Notably, a significantly larger number of studies used PL, traditionally regarded as a stable, xeno-free product, with less homogeneity when compared to FBS and vastly used in the mass ex vivo expansion of BM-MSCs for clinical applications (Schallmoser et al., 2020). However, a recent study by Scherer et al. (2012) comparing activated and non-activated platelets demonstrated better tissue healing and angiogenesis in the non-activated platelet group. The authors suggested that natural activation occurring intrinsically 'on demand' may be more effective than a bolus of exogenously activated platelets (Scherer et al., 2012). As yet, no in vitro studies have compared the effects of activated and non-activated PPs on the biophysiological functions of human BM-MSCs, which could guide clinical practice on whether these PPs should be activated ex vivo prior to delivery.

Another important aspect to consider is whether leukocyte content in PP would influence the biophysiological behaviour of BM-MSCs. P-PRP$\mathrm{CM}$ was found to result in stronger osteogenic gene expression in BM-MSCs (Yin et al., 2016). However, the effects of leukocyte content on proliferation, differentiation and migration remain unclear, due to the conflicting findings from the small number of studies available (Moisley et al., 2019; Perut et al., 2013; Yin et al., 2016). Furthermore, the effect of leukocyte content on other functions of BM-MSCs, such as cytokine expression and immunomodulation, were not assessed.
Expansion of harvested BM-MSCs and the subsequent use of subcultured (or passaged) BMMSCs were common in most studies (Agis et al., 2009; Amable et al., 2014; Bernardi et al., 2017; Bernardi et al., 2013; Dohan Ehrenfest et al., 2010; Goedecke et al., 2011; Gottipamula et al., 2012; Gruber et al., 2004; Infante et al., 2017; Jonsdottir-Buch et al., 2013; Kosmacheva et al., 2014; Lange et al., 2012; Liou et al., 2018; Lucarelli et al., 2003; Lucarelli et al., 2010; Moisley et al., 2019; Moradian et al., 2017; Muraglia et al., 2015; Murphy et al., 2012; Nguyen et al., 2019; Parsons et al., 2008; Prins et al., 2009; Samuel et al., 2016; Schar et al., 2015; Skific et al., 2018; Verrier et al., 2010; Vogel et al., 2006; Yin et al., 2016), since larger quantities of BM-MSCs were desired for laboratory assays. However, studies have demonstrated the 'internalisation' of bovine proteins in BM-MSCs expanded in culture medium containing FBS, a commonly used animal serum (Spees et al., 2004). The stemness of passaged BM-MSCs and, therefore, how closely it reflects fresh BM-MSCs used in clinical practice is questionable. Furthermore, in vitro passage of BM-MSCs could also result in the loss of homing (Rombouts and Ploemacher, 2003), migration (Bustos et al., 2014; Choumerianou et al., 2010), proliferation (Ganguly et al., 2017) and differentiation (Kim et al., 2012) capabilities of BM-MSCs. The use of fresh or minimally cultivated BM-MSCs would have been more representative of clinical practice.

Incubation time with PP reflects the duration which BM-MSCs were exposed to the inductive molecules contained in $\mathrm{PP}$, such as growth factors and cytokines (Di Matteo et al., 2015; Roffi et al., 2017). BM-MSCs in most of the studies were exposed to a continuous dose of PP through prolonged periods of co-incubation. Differently, in clinical practice, autologous PPs and BM-MSCs are commonly harvested on the day of surgery and incubated for a short duration prior to implantation (Lee et al., 2014; Martin et al., 2013). In vitro studies reflecting the short incubation periods seen in clinical settings would have been more informative, as it will elucidate whether short PP exposure is effective at optimising the potent biophysiological functions of BM-MSCs necessary for advantageous bone repair.

Despite the inherent weaknesses limiting transferability into clinical practice, these in vitro studies in humans have nonetheless demonstrated promising findings. Most in vitro studies focused on the impact of PP on proliferation and differentiation of BM-MSCs (Amable et al., 2014; Ben Azouna et al., 2012; Bernardi et al., 2017; Bernardi et al., 2013; Dohan Ehrenfest et al., 2010; Goedecke et al., 2011; Gottipamula et al., 2012; Gruber et al., 2004; Infante et al., 2017; Jenhani et al., 2011; Jonsdottir-Buch et al., 2013; Karadjian et al., 2020; Kosmacheva et al., 2014; Lange et al., 2012; Liou et al., 2018; Lucarelli et al., 2003; Lucarelli et al., 2010; Moisley et al., 2019; Moradian et al., 2017; Muraglia et al., 2014; Muraglia et al., 2015; Murphy et al., 2012; Nguyen et al., 2019; Parsons et al., 
2008; Perut et al., 2013; Prins et al., 2009; Samuel et al., 2016; Skific et al., 2018; Verrier et al., 2010; Vogel et al., 2006; Yin et al., 2016). The huge focus thrown upon these two biophysiological functions were perhaps unsurprising, given that the successful proliferation of BM-MSCs and its subsequent differentiation into the desired mesenchymal lineage are key determinants for successful bone repair. Overall, PP has been shown to increase cellular proliferation (Amable et al., 2014; Ben Azouna et al., 2012; Bernardi et al., 2013; Dohan Ehrenfest et al., 2010; Goedecke et al., 2011; Gottipamula et al., 2012; Gruber et al., 2004; Infante et al., 2017; Jenhani et al., 2011; JonsdottirBuch et al., 2013; Karadjian et al., 2020; Lucarelli et al., 2003; Moradian et al., 2017; Muraglia et al., 2014; Muraglia et al., 2015; Murphy et al., 2012; Nguyen et al., 2019; Perut et al., 2013; Prins et al., 2009; Samuel et al., 2016; Skific et al., 2018; Yin et al., 2016) and osteogenic differentiation of BM-MSCs in the majority of these studies (Amable et al., 2014; Dohan Ehrenfest et al., 2010; Gottipamula et al., 2012; Karadjian et al., 2020; Kosmacheva et al., 2014; Lucarelli et al., 2003; Parsons et al., 2008; Perut et al., 2013; Samuel et al., 2016; Skific et al., 2018; Verrier et al., 2010; Yin et al., 2016). The use of passaged BM-MSCs, known to affect differentiation potential (Kim et al., 2012), could explain these mixed findings in the remaining studies [no difference $(n=3)$ (Ben Azouna et al., 2012; Goedecke et al., 2011; Prins et al., 2009); reduced $(n=1)$ (Vogel et al., 2006); equivalent $(n=2)$ (Gruber et al., 2004; Jonsdottir-Buch et al., 2013)], therefore, warranting further research using fresh or minimally cultured BM-MSCs. On the other hand, chondrogenic and adipogenic differentiation were not improved by PPs in most studies (Amable et al., 2014; Ben Azouna et al., 2012; Goedecke et al., 2011; Gottipamula et al., 2012; Lange et al., 2012; Liou et al., 2018; Prins et al., 2009; Vogel et al., 2006). Increasing PP concentrations up to $10 \%$ have also been demonstrated to improve proliferation (Amable et al., 2014; Bernardi et al., 2017; Bernardi et al., 2013; Goedecke et al., 2011; Gottipamula et al., 2012; Jenhani et al., 2011; Karadjian et al., 2020; Lucarelli et al., 2003; Muraglia et al., 2014; Muraglia et al., 2015; Murphy et al., 2012; Nguyen et al., 2019; Perut et al., 2013; Prins et al., 2009; Skific et al., 2018; Yin et al., 2016). However, no studies have yet investigated the correlation between PP concentration and the differentiation potential of BM-MSCs.

The ability to migrate is a potent biophysiological function possessed by BM-MSCs, allowing it to be signalled and recruited to areas requiring more osteogenic cells (Su et al., 2018). Hence, being able to influence its migratory capacity is crucial, thereby improving the chances of successful bone repair. PP represents such a promising chemotactic stimulus since it possesses multiple osteoinductive molecules (Di Matteo et al., 2015; Roffi et al., 2017; Schallmoser et al., 2020). This was further confirmed by the improved migratory capacity in BM-MSCs exposed to PP (Goedecke et al., 2011; Gruber et al., 2004; Infante et al., 2017; Moisley et al., 2019; Murphy et al., 2012; Nguyen et al., 2019; Schar et al., 2015; Yin et al., 2016). The effects of PP concentration were compared up to $10 \%$ only, whereby increasing PP concentrations enhances the migratory capacity of BM-MSCs (Goedecke et al., 2011; Gruber et al., 2004; Murphy et al., 2012; Nguyen et al., 2019).

Comminuted, segmental and open fractures represent injuries with a higher risk of impaired bone healing. This is because the zone of injury in these high-risk fractures are subjected to immense amounts of inflammatory, oxidative and apoptotic stressors (Wang et al., 2017). Despite the paucity of research on immunomodulation and response towards apoptotic stress, findings from Yin et al. (2016) were encouraging. Yin et al. (2016) demonstrated PRP-CM to (i) have a protective effect and preserved the viability of BMMSCs in the face of apoptotic stress and (ii) improve immunomodulatory functions in BM-MSCs. Despite the use of subcultured BM-MSCs and PPs that were not donor-matched, it has nonetheless uncovered important biophysiological functions of BM-MSCs primed by PPs relevant to improving bone repair in high-risk fractures. Therefore, further research using fresh donor-matched BM-MSCs and PP is warranted given it bears closer resemblance to clinical practice.

An important question to ask when applying these biologics during surgery is whether the priming effect of PPs on BM-MSCs observed in in vitro studies would be lost following surgery, since it is subjected to the homeostatic and dilutional effects of circulation once inside the human body. Albeit not assessed by many studies, results from Lucarelli et al. (2003) were encouraging, where BM-MSCs were demonstrated to retain their improved osteogenic and chondrogenic capability despite PP withdrawal. This suggested that the priming effect of PPs is perhaps not transient and limited to the incubation period only, as could be expected.

In the attempt to uncover the underlying cellular and molecular mechanisms explaining the effects of PP on the bio-physiology of BM-MSCs, several studies have investigated the release of cytokines, growth factors and proteins. Following PP exposure, BM-MSCs were found to upregulate their release of (i) pro-inflammatory cytokines (IL-2R, IL-6, IL-7, IL-8, IL-12, IL-15, PGE2, NO) (Amable et al., 2014; Ben Azouna et al., 2012; Jenhani et al., 2011; Yin et al., 2016); (ii) chemokines (eotaxin, IP-10, MIP-1 $\beta$, MCP-1, RANTES, SDF-1 $\alpha$ ) (Amable et al., 2014; Ben Azouna et al., 2012; Goedecke et al., 2011; Jenhani et al., 2011); (iii) pro-angiogenic factors (thrombospondin, PLGF, aFGF, VEGF-D) (Amable et al., 2014) and (iv) osteogenic proteins (osteocalcin, RUNX2 and BMP2) (Samuel et al., 2016; Verrier et al., 2010; Yin et al., 2016). All of these are important for successful bone repair, since higher levels of pro-inflammatory cytokines advantageous for mediating the early inflammatory phase of fracture healing, whilst inducing an angiogenic and chemotactic state in BM-MSCs, lead to improved vascularity and recruitment of nonresident BM-MSCs, cytokines and growth factors to 


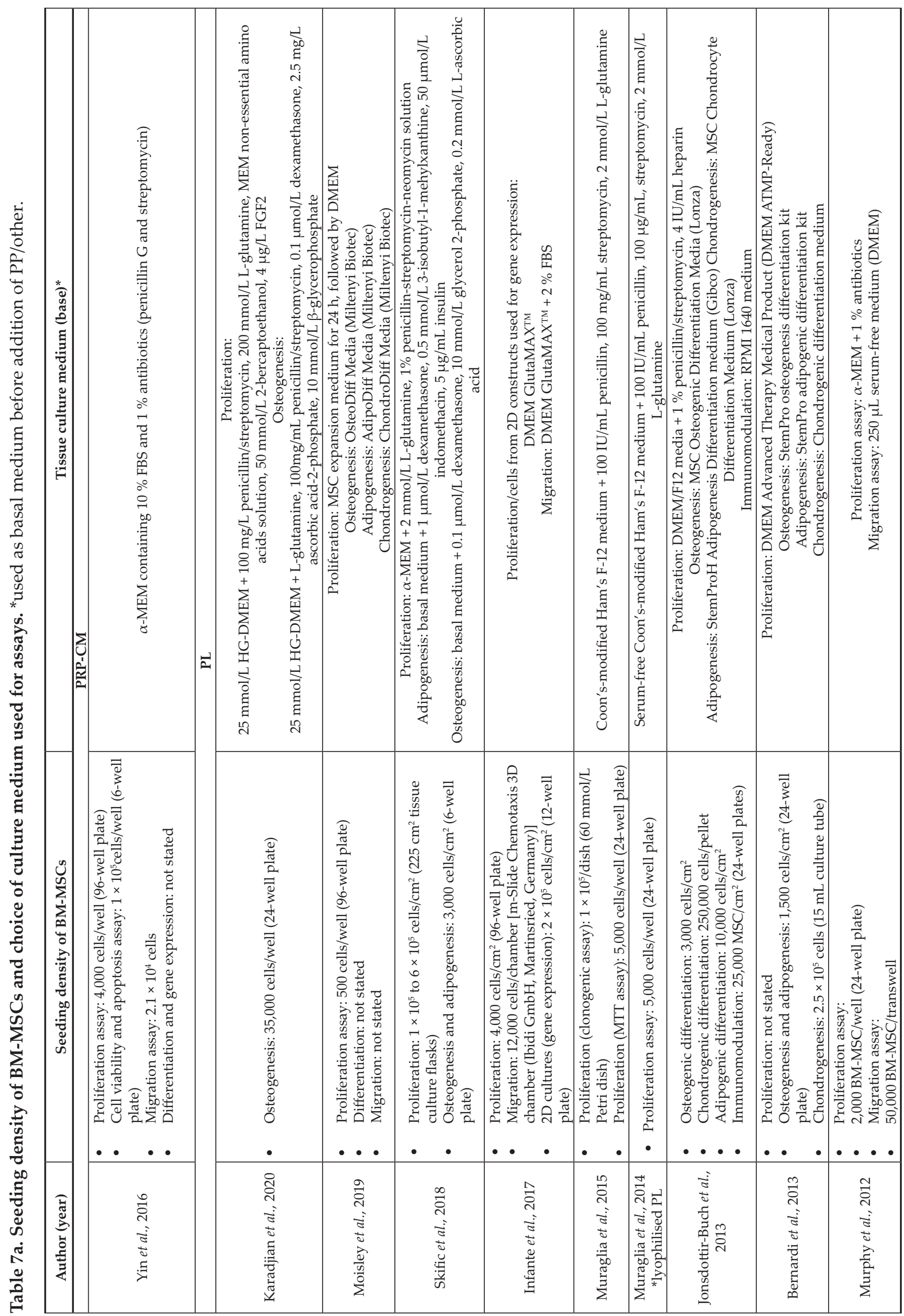




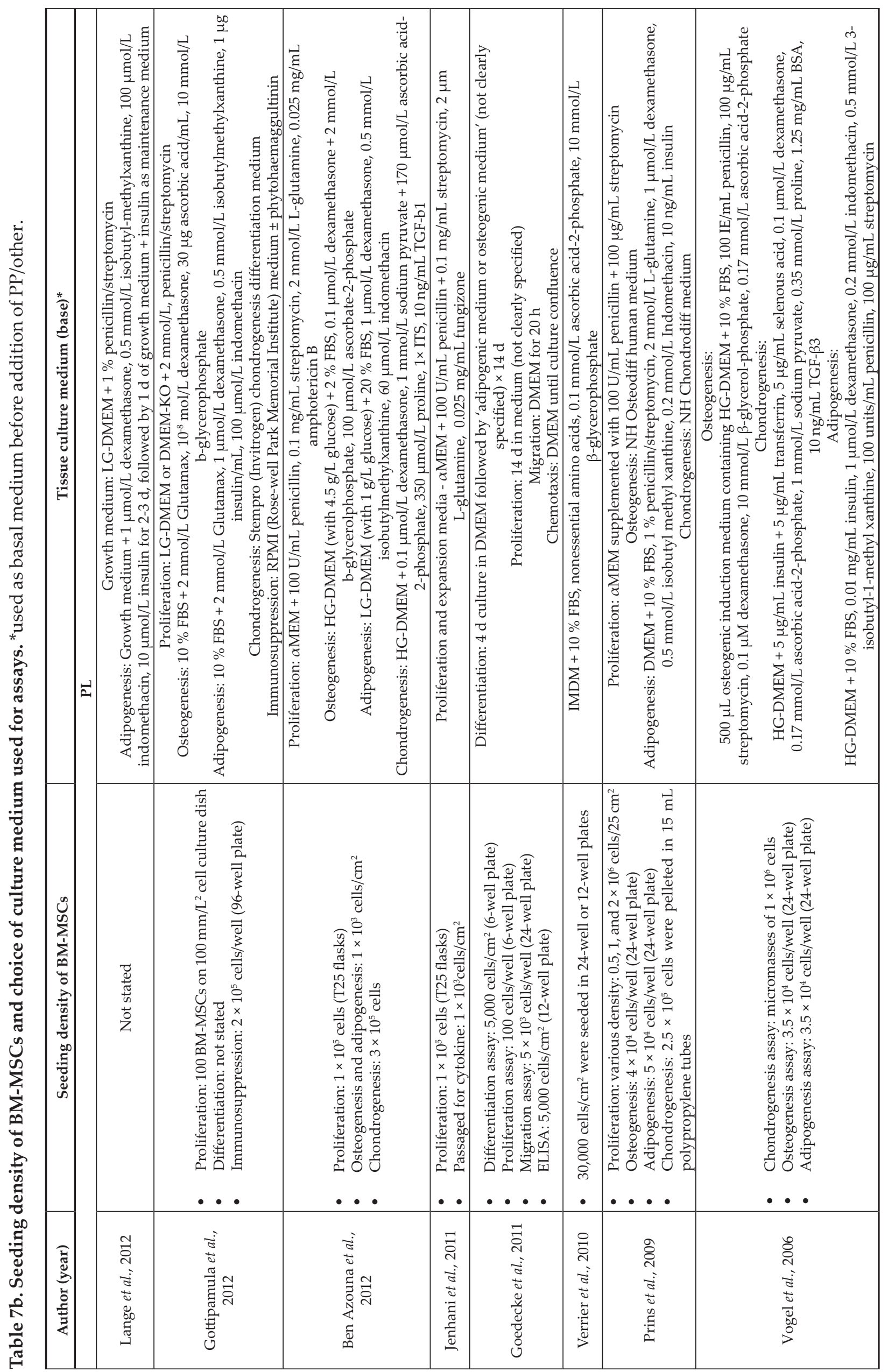




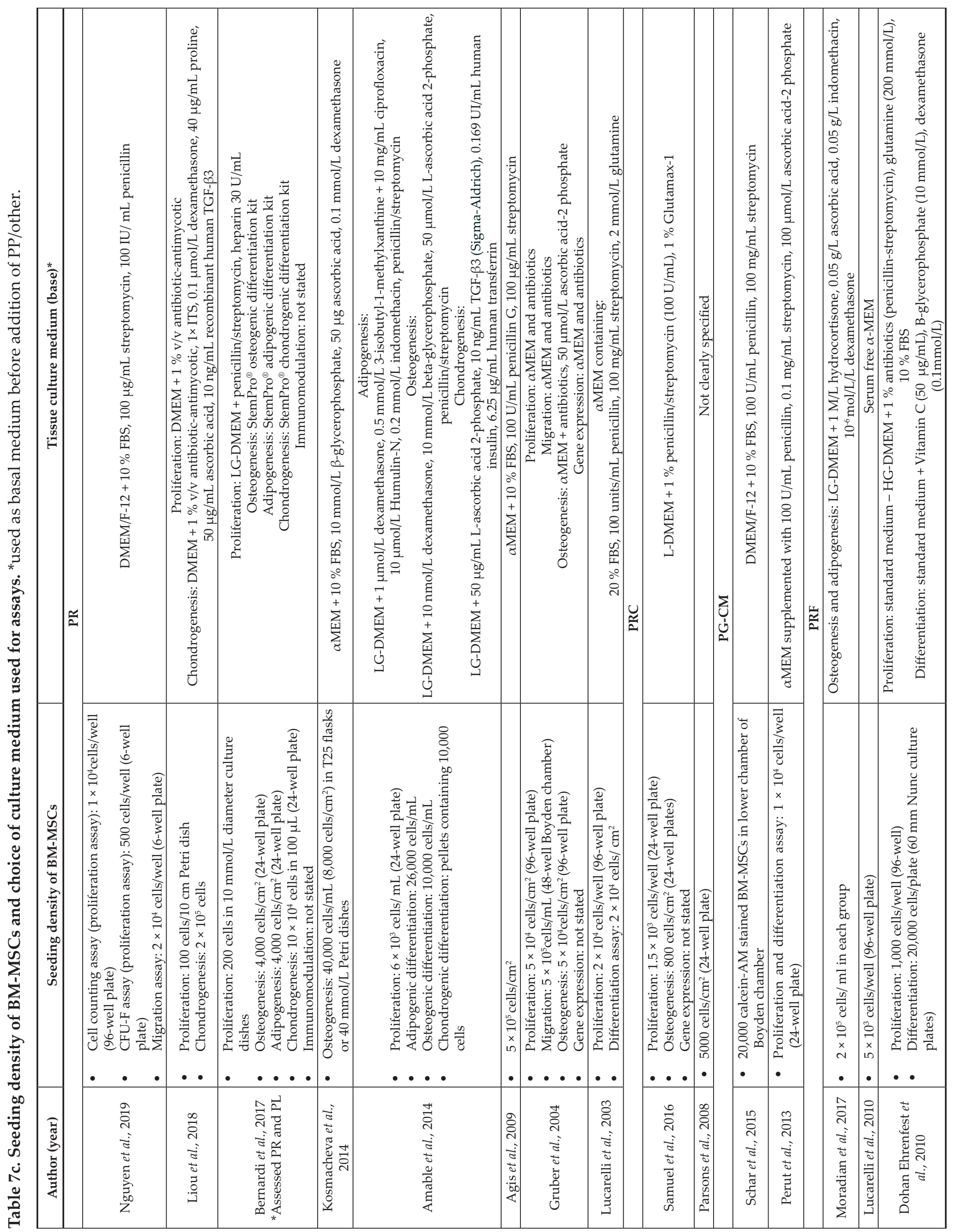




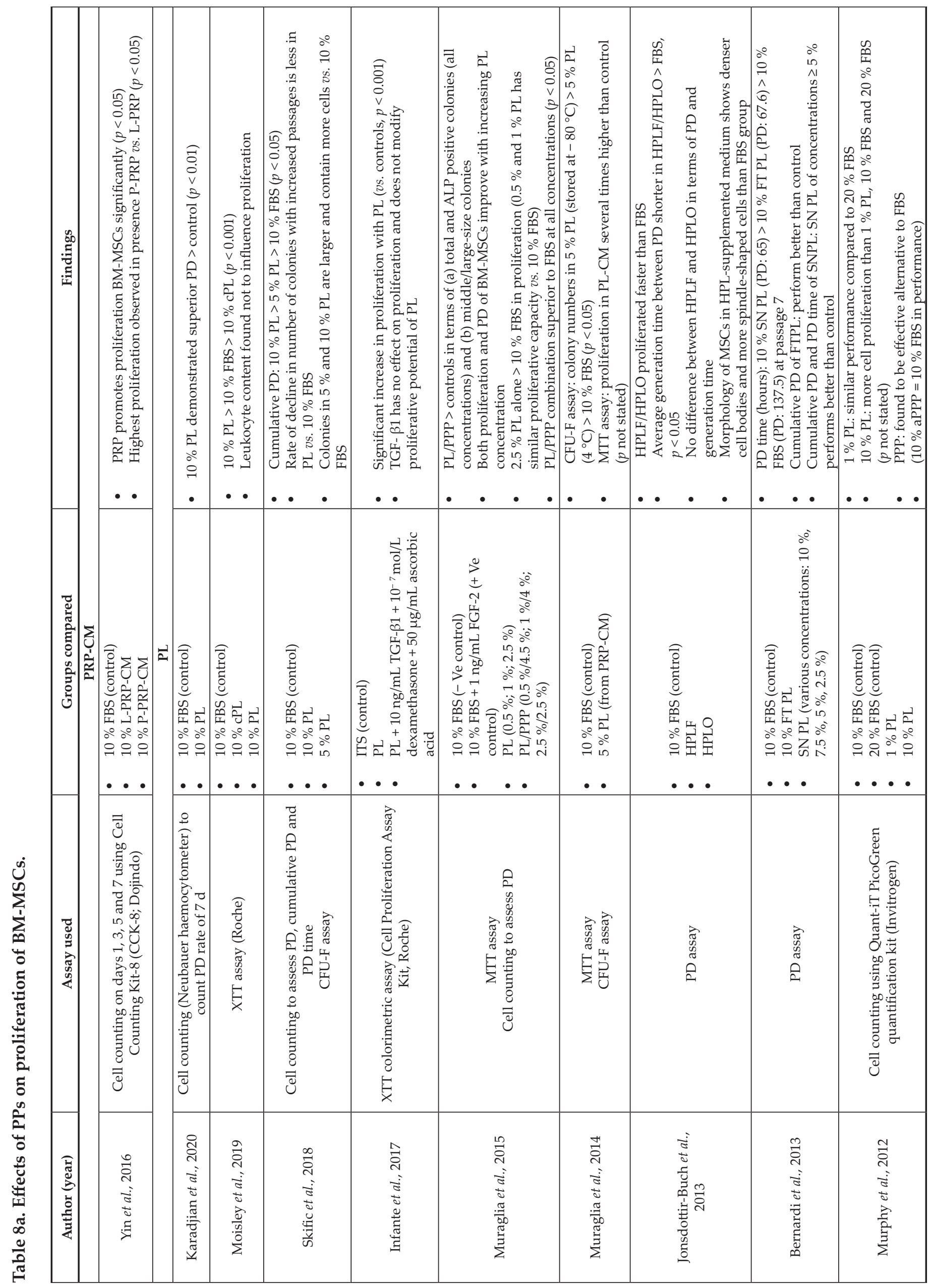




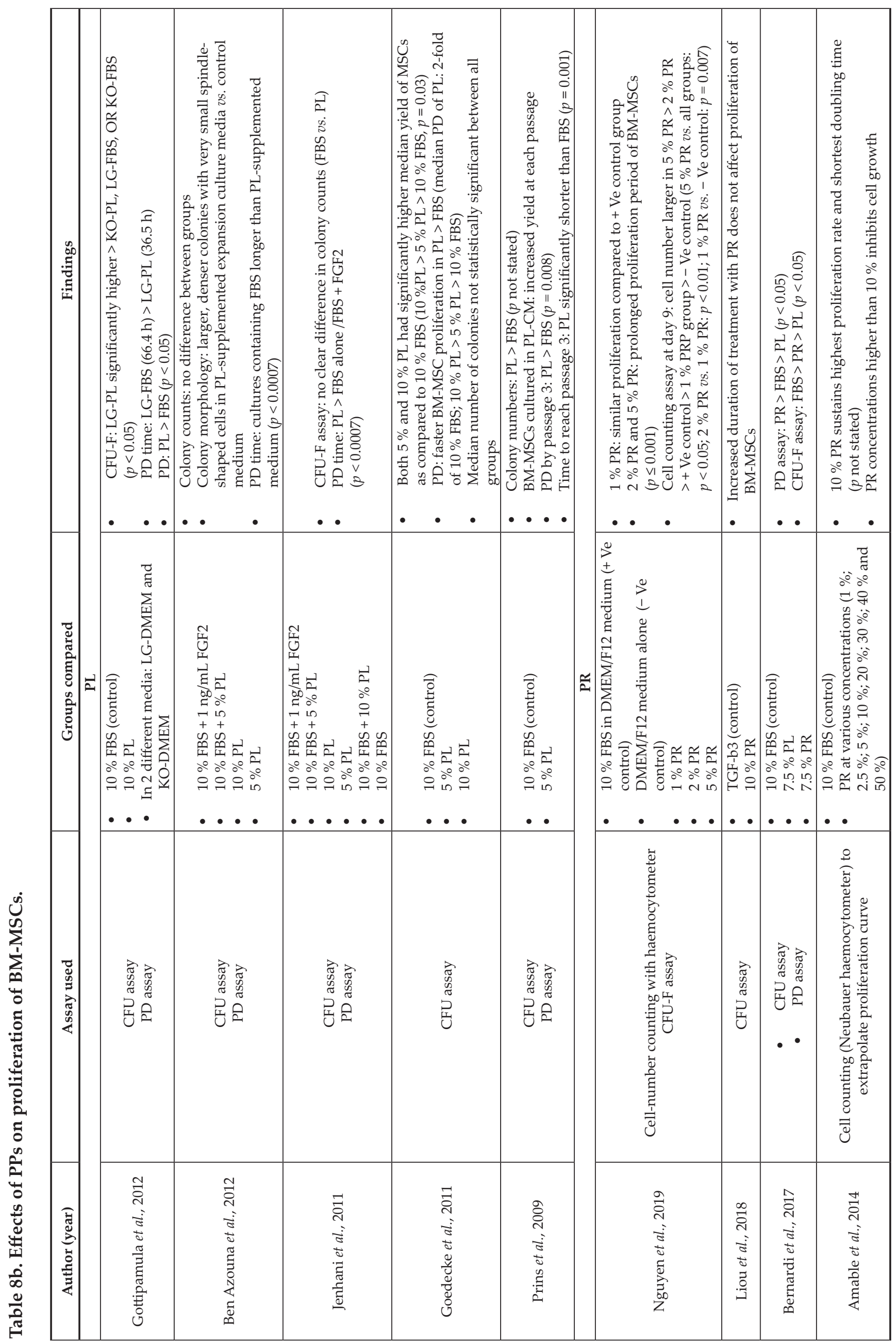




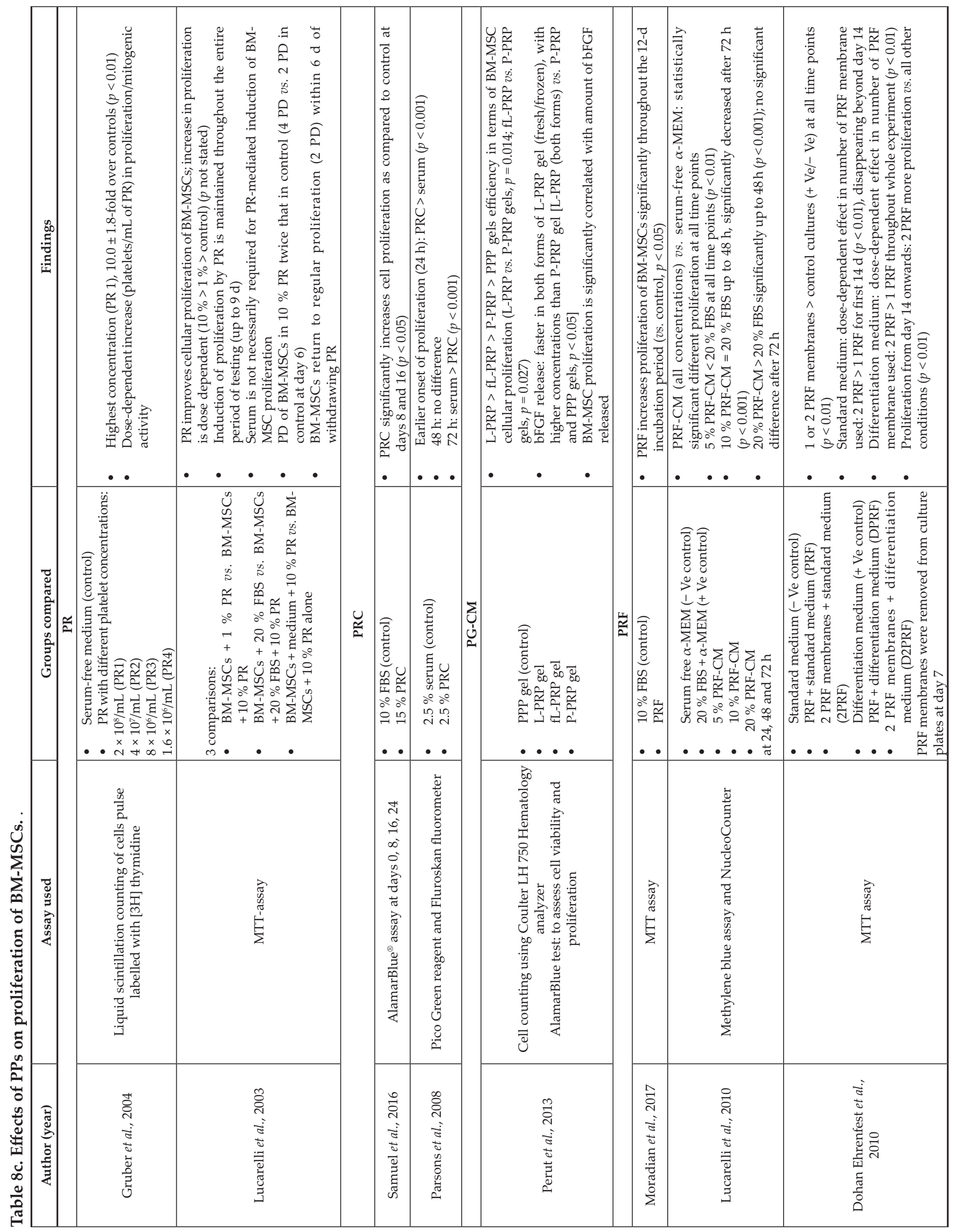




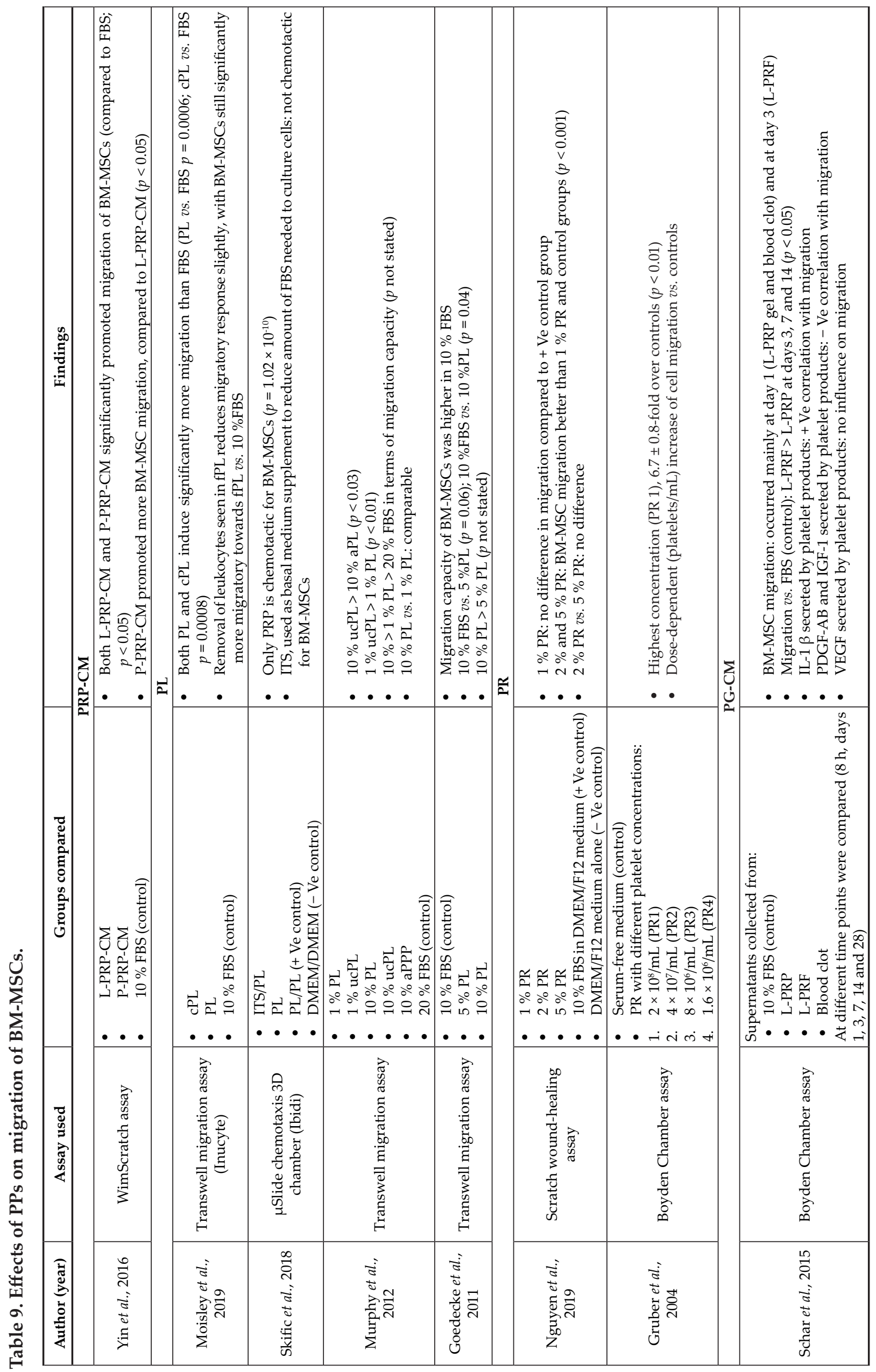




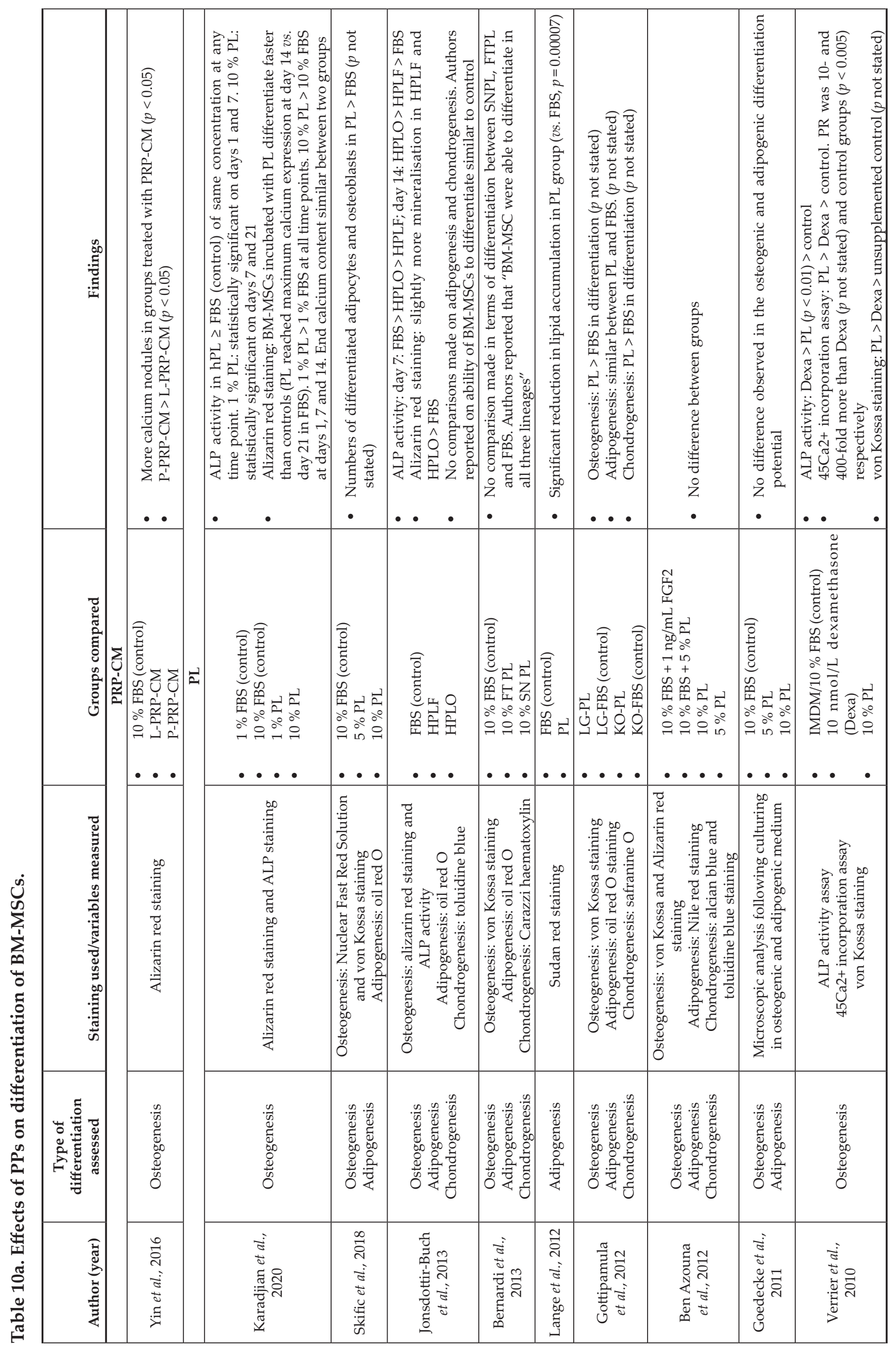




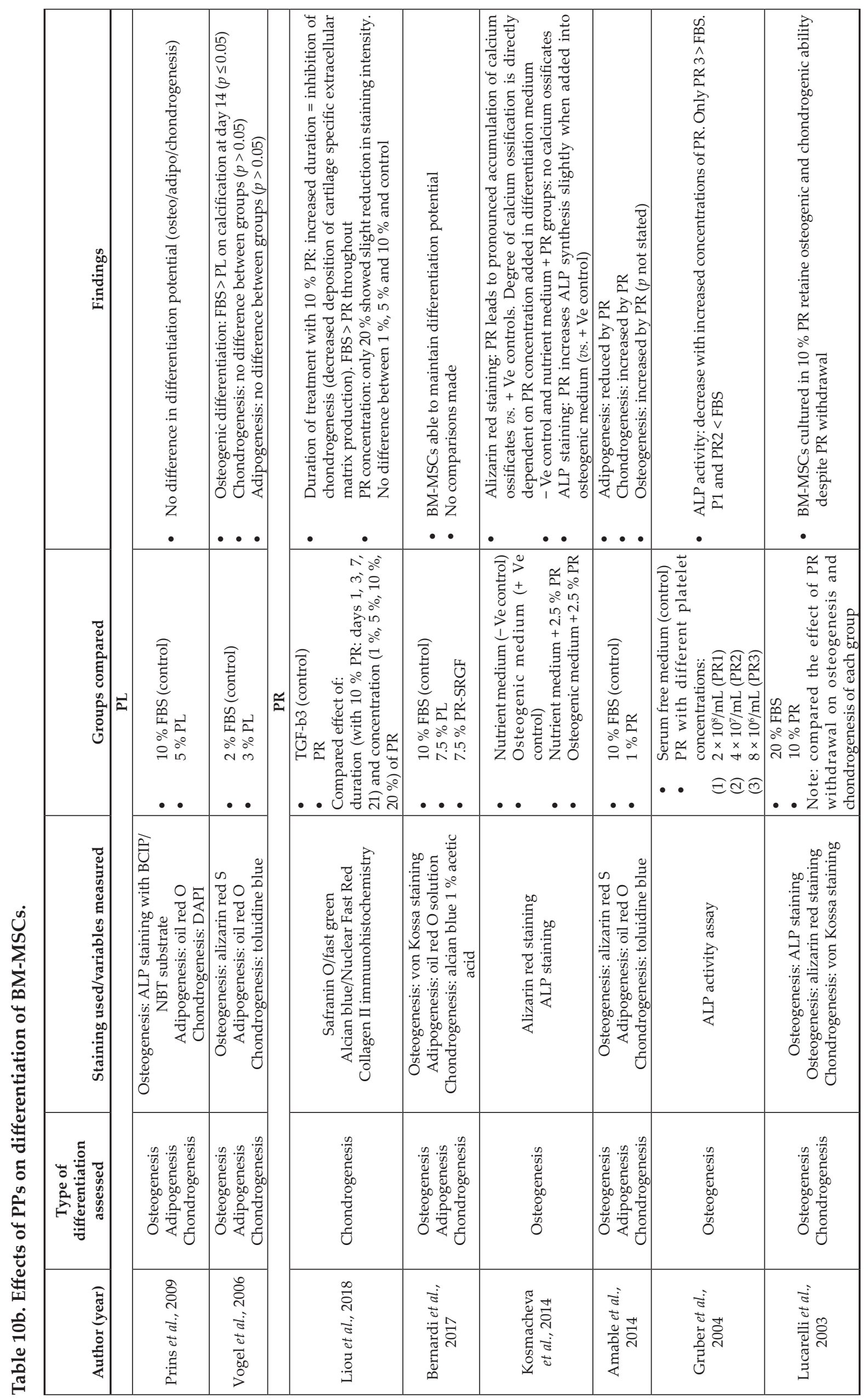




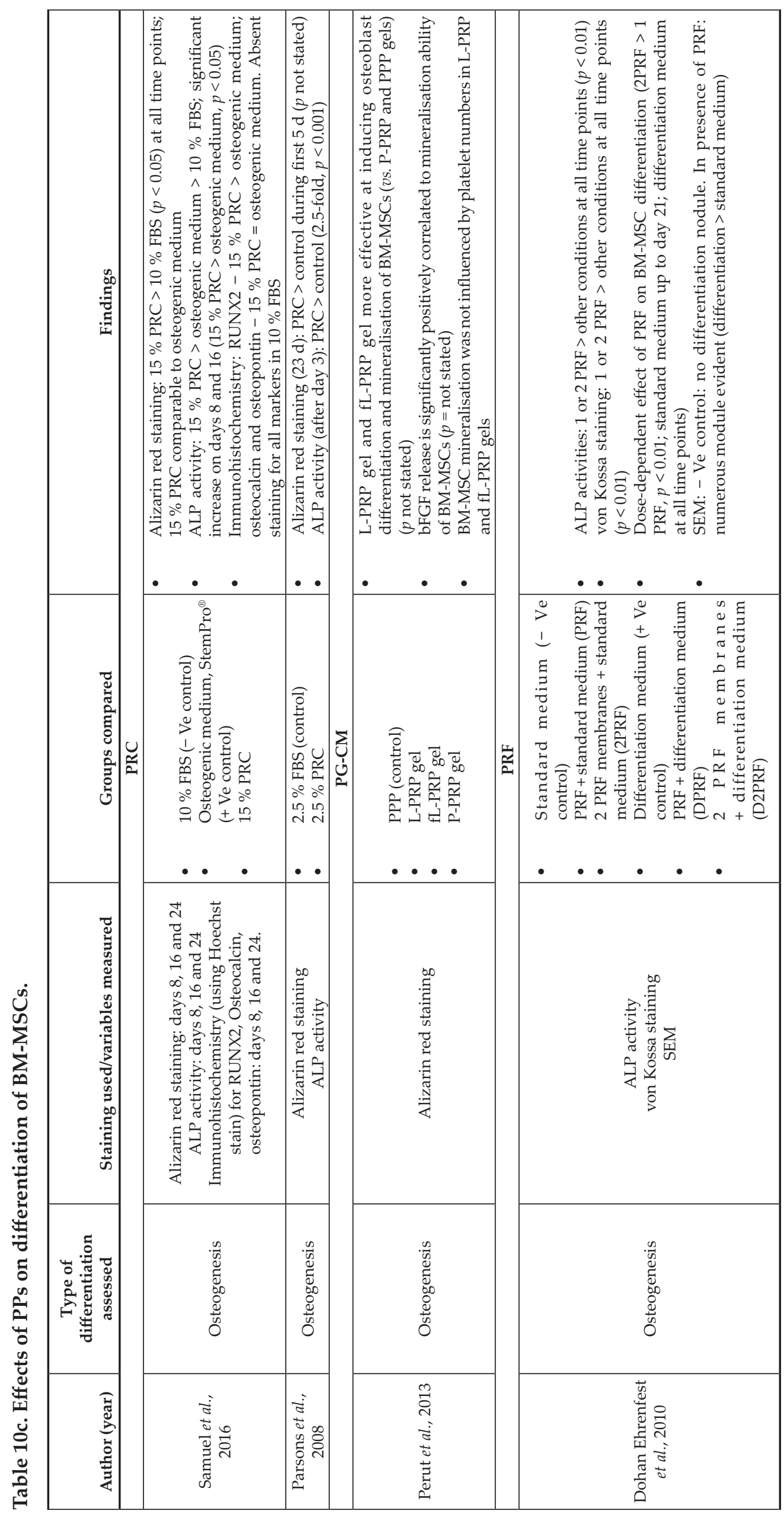




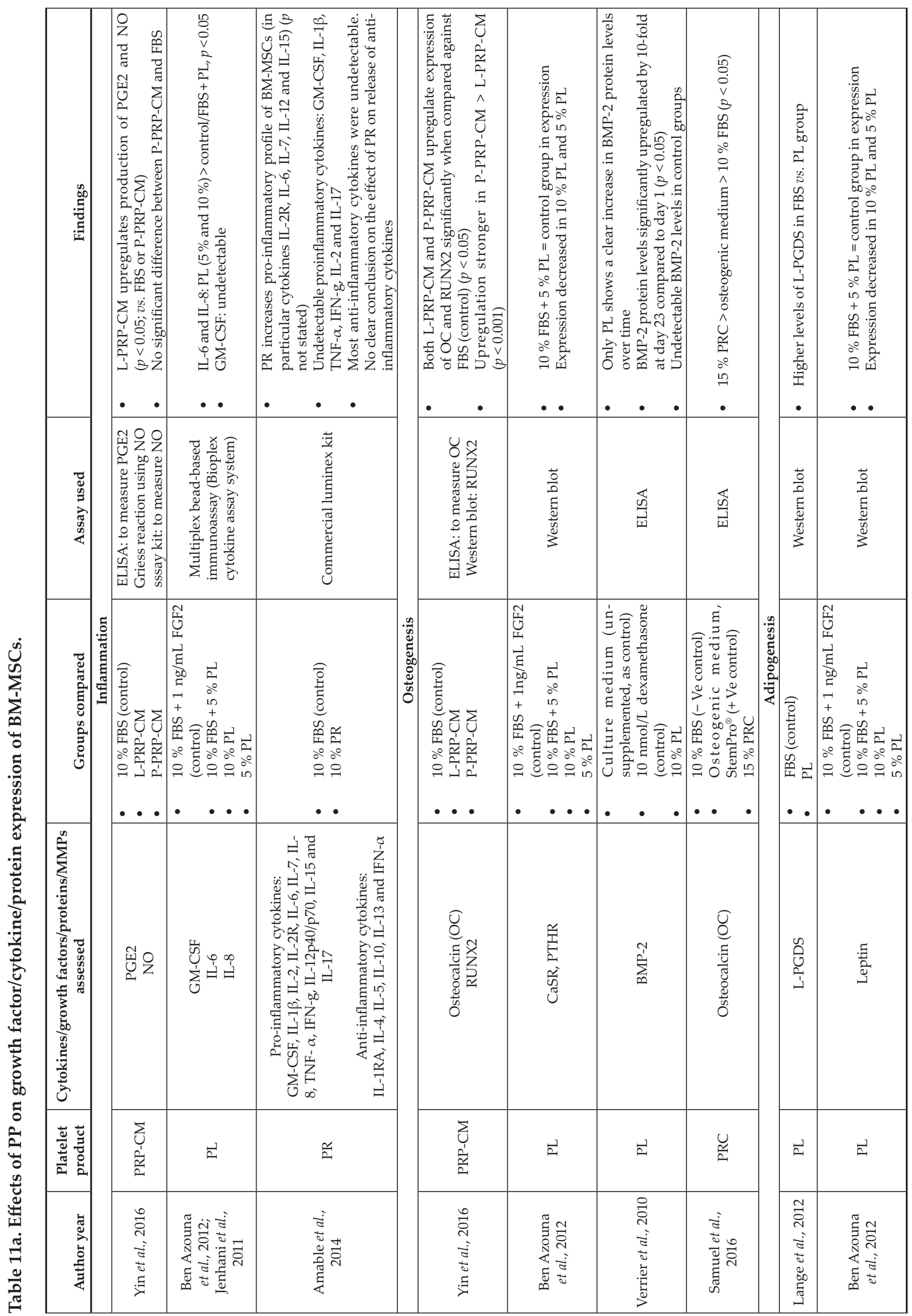




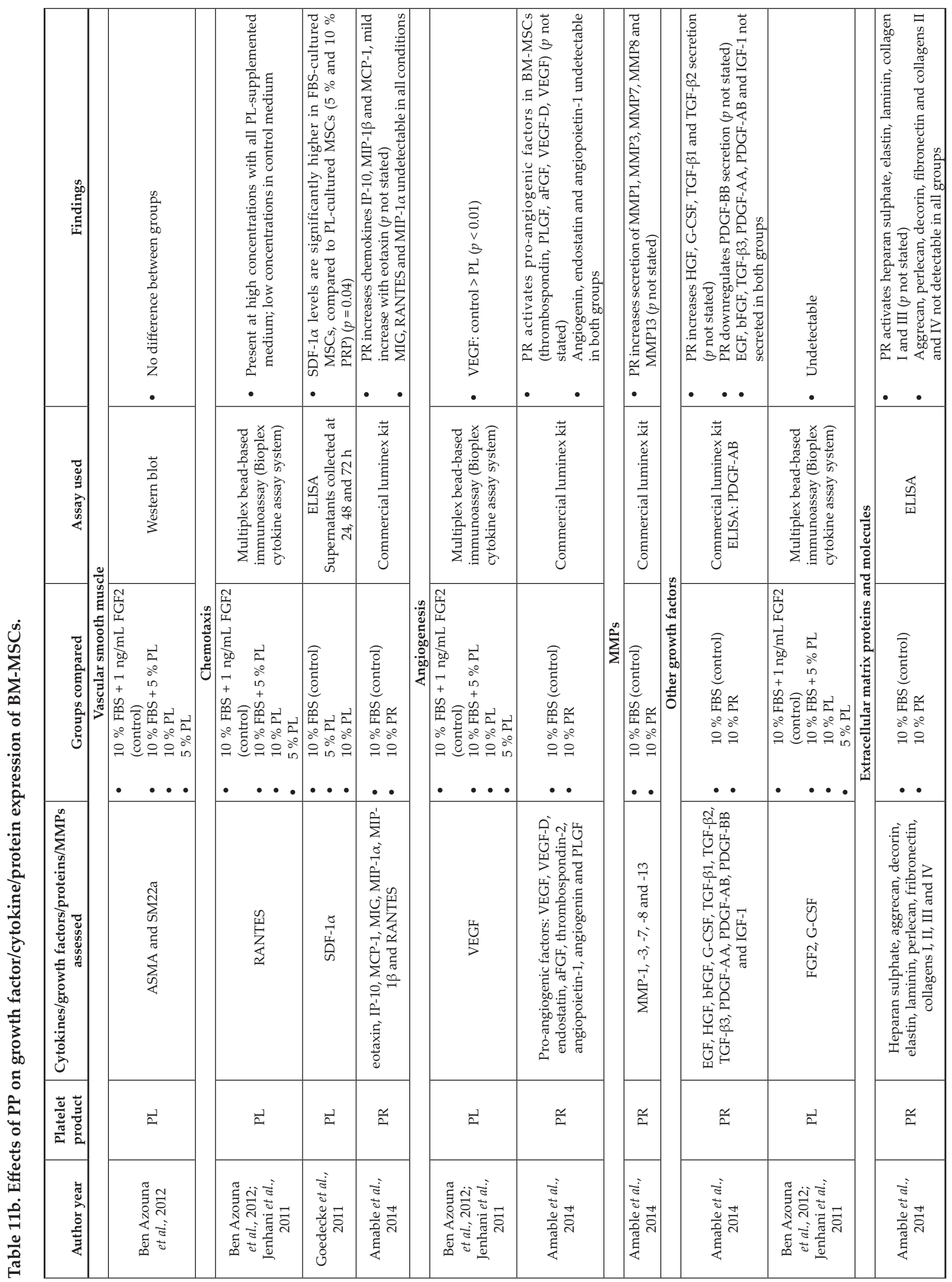




\begin{tabular}{|c|c|c|c|c|c|c|c|}
\hline 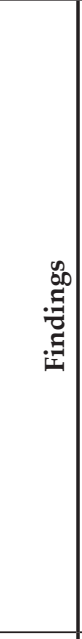 & 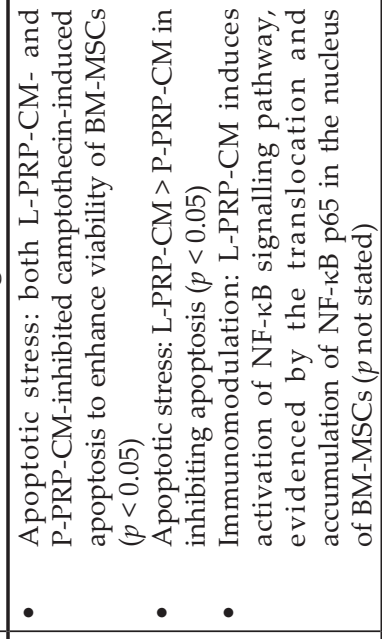 & 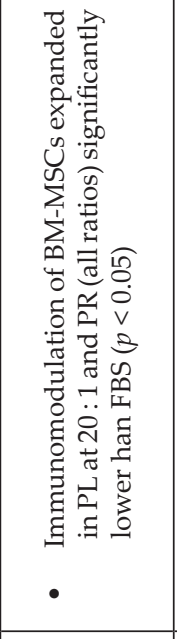 & 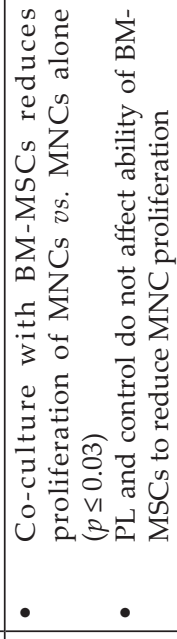 & 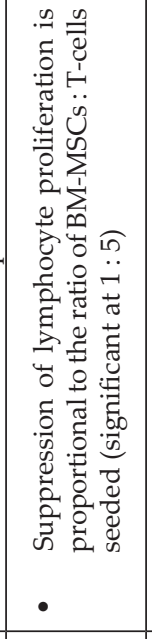 & 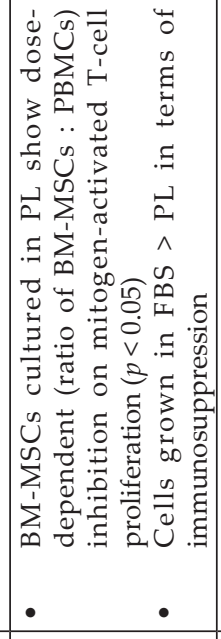 & 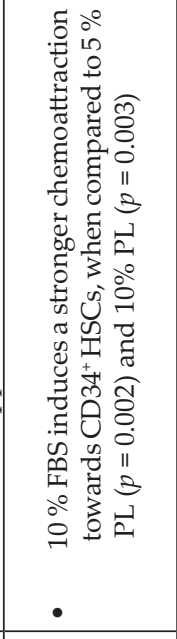 & 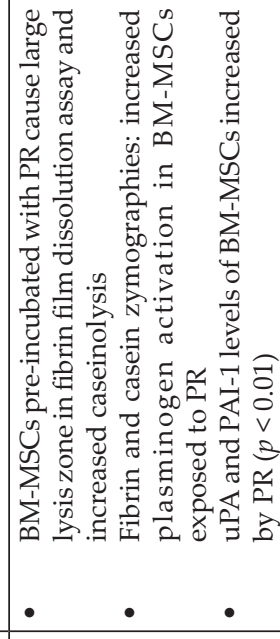 \\
\hline 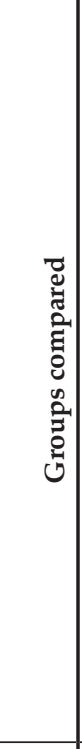 & 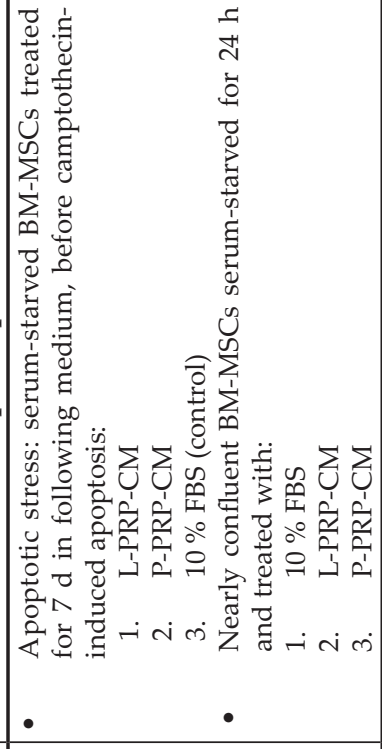 & 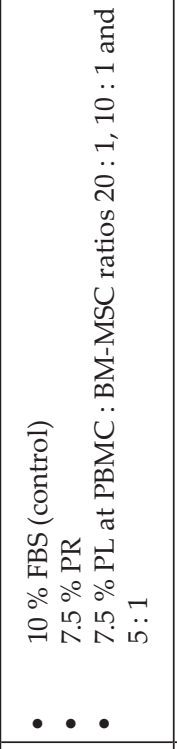 & 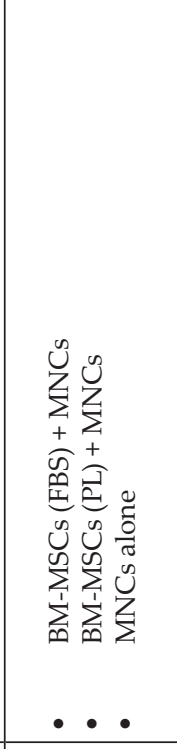 & 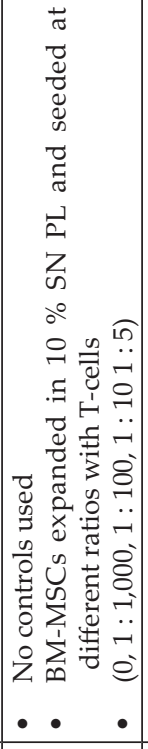 & 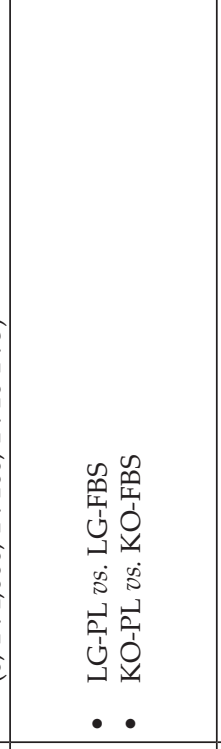 & 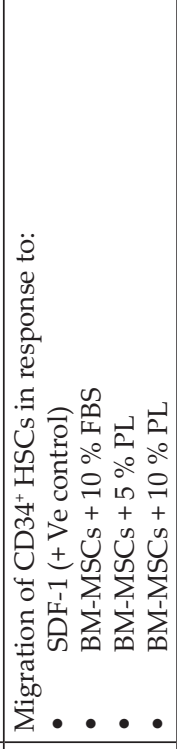 & 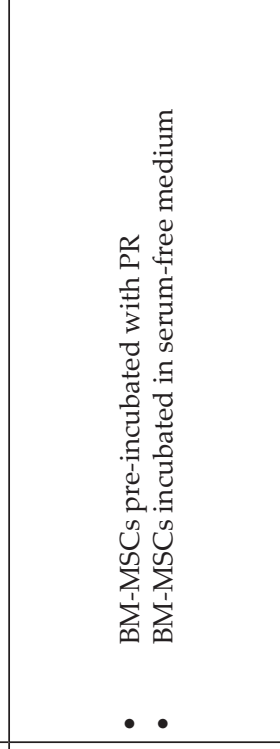 \\
\hline 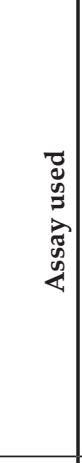 & 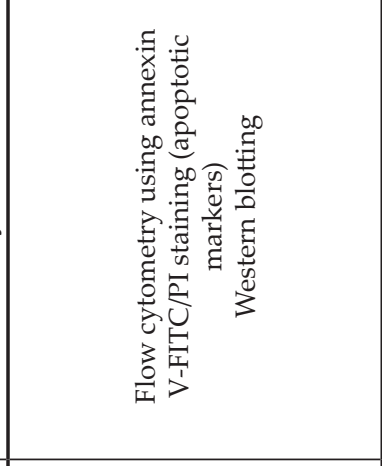 & 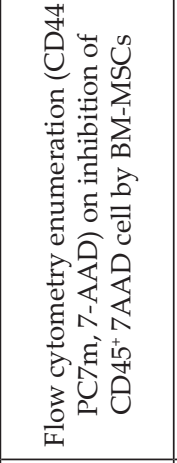 & 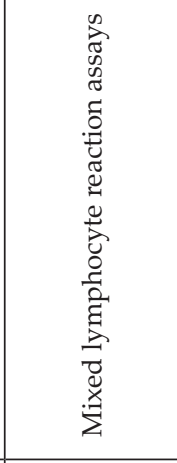 & 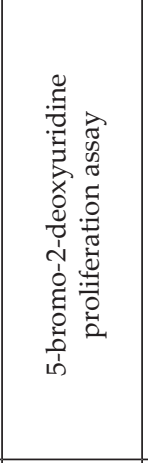 & 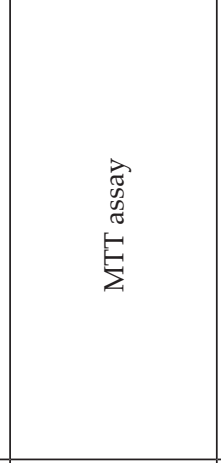 & 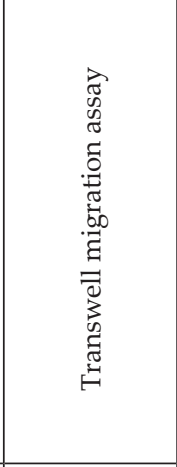 & 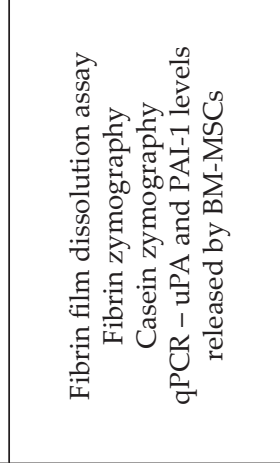 \\
\hline 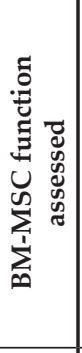 & 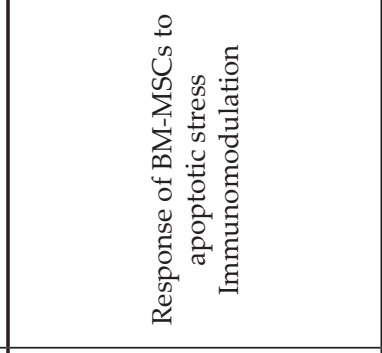 & 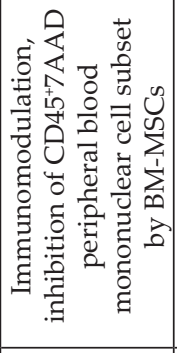 & 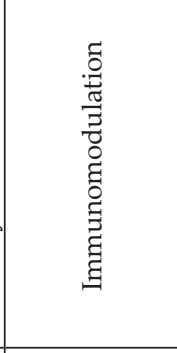 & 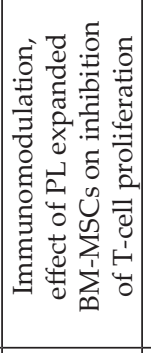 & 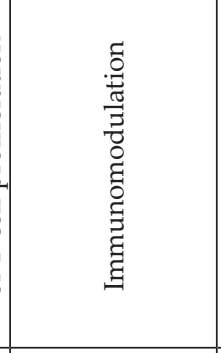 & 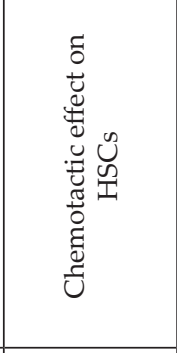 & 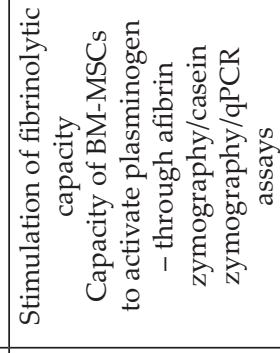 \\
\hline 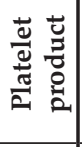 & 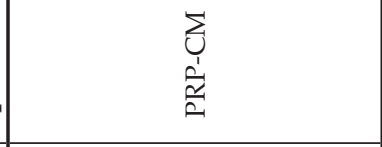 & 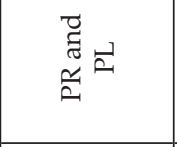 & 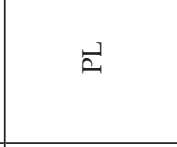 & $\overrightarrow{2}$ & $\vec{E}$ & $\overrightarrow{2}$ & 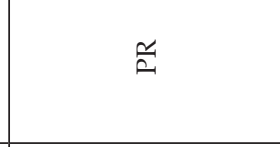 \\
\hline 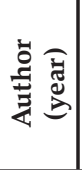 & 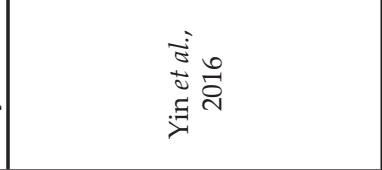 & 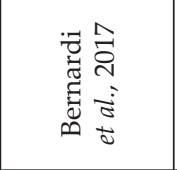 & 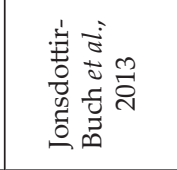 & 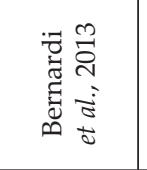 & 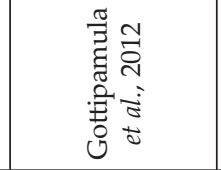 & 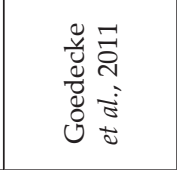 & 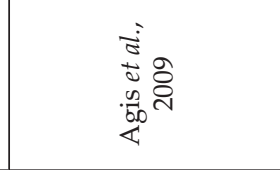 \\
\hline
\end{tabular}




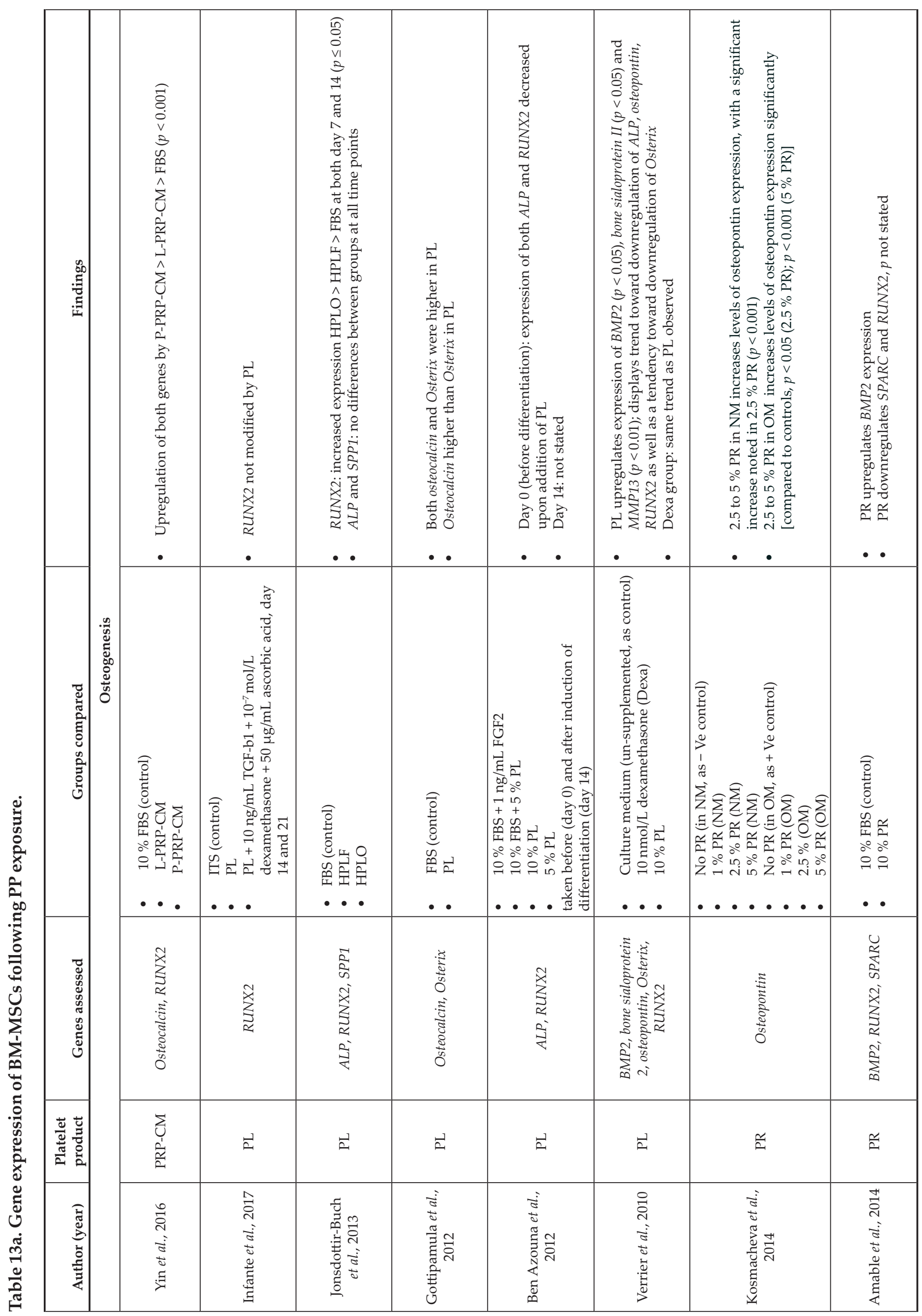




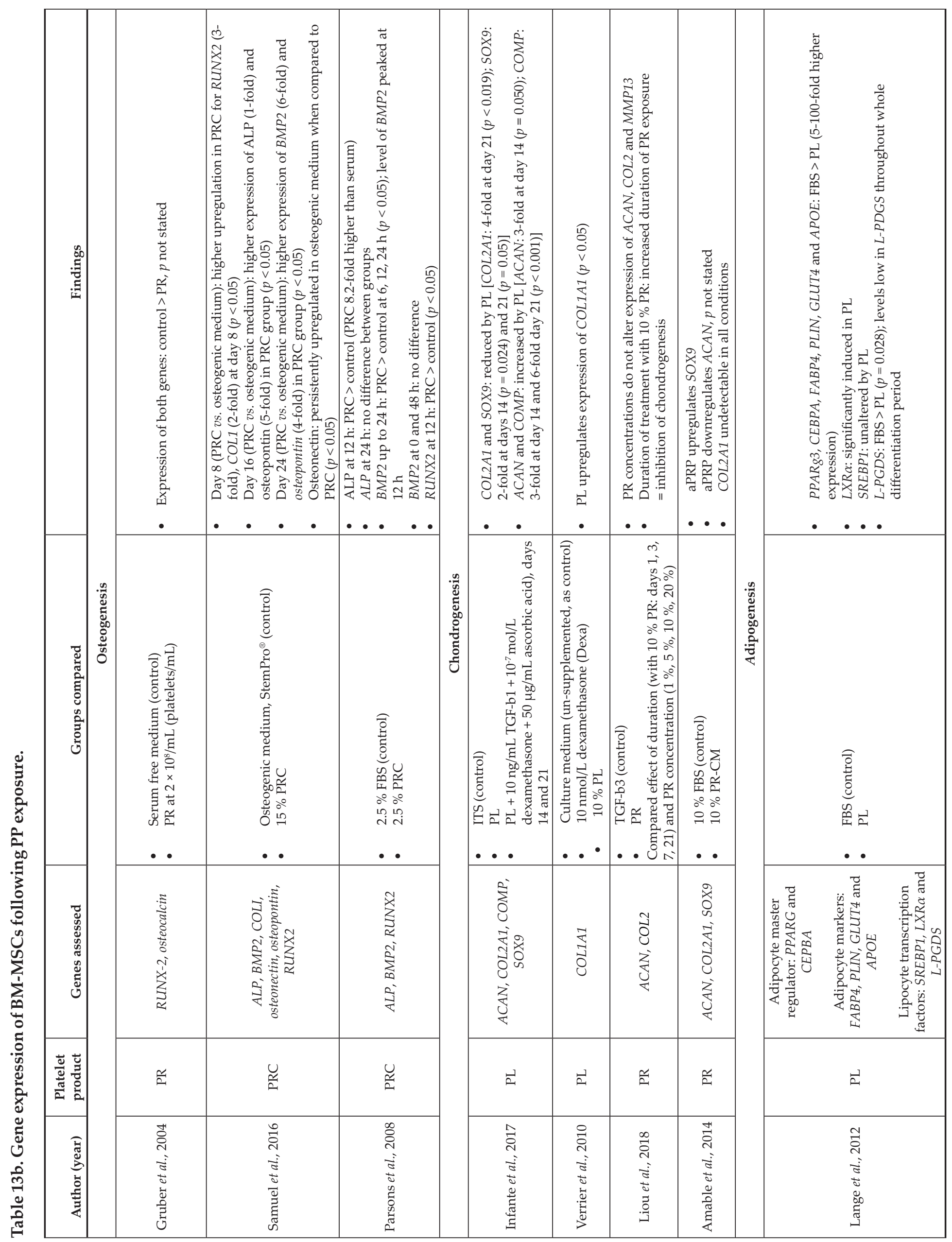




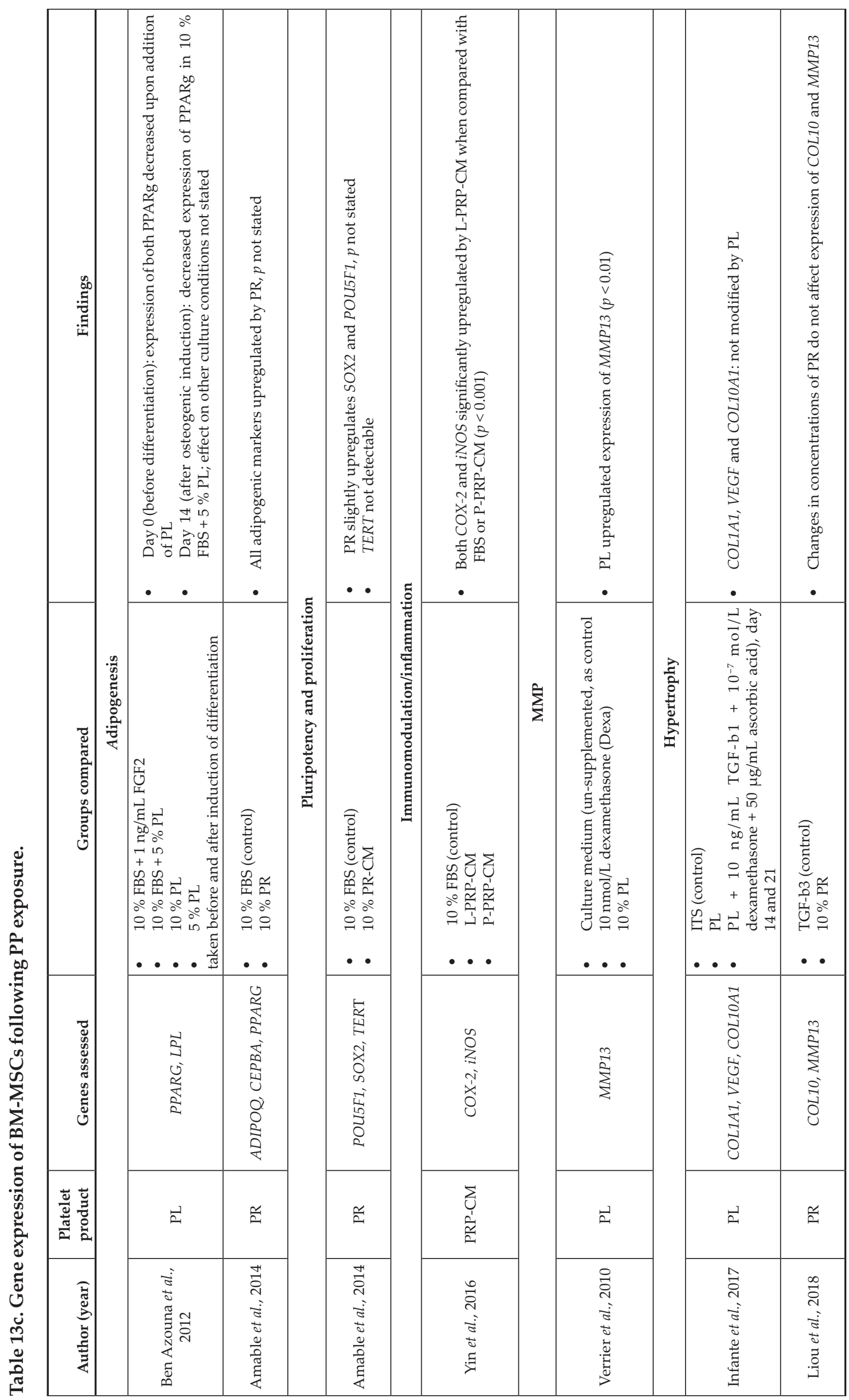


the zone of injury - further guaranteeing successful bone repair. Moreover, the release of growth factors (HGF, G-CSF, TGF- 1 1, TGF- $\beta 2$ ), MMPs (MMP1, MMP3, MMP7, MMP8, MMP13) and extracellular matrix molecules (heparan sulphate, elastin, laminin, collagen I and III), with multiple regulatory roles that enhance bone regeneration, was likewise larger in BM-MSCs exposed to PP (Amable et al., 2014). Taken together, these results agreed with findings from functional assays.

BM-MSC gene expression has also been assessed by several studies. Proliferative gene markers in BM-MSCs were found to be consistently upregulated following PP exposure, confirming the findings from differentiation assays (Amable et al., 2014). The effects of PP on the expression of BM-MSC gene markers for osteogenesis, chondrogenesis, adipogenesis and immunomodulation were rather variable and dependant on the gene assessed. Alterations to the physiological behaviour and characteristics of subcultured BM-MSCs following multiple passages using highly variable media (Ganguly et al., 2019) could potentially explain the inconsistency in gene expression observed.

The present systematic review highlights the usefulness of PPs, many of which could be harvested and delivered autologously. Until research would advance in a manner whereby the array of most potent growth factors, cytokines and proteins could be commercially produced and compliant with the Good Manufacturing Practice, PPs remain a useful adjunct to bone healing. To ensure that the strengths of these PPs are put to the best use and not risk them being disregarded in the history of medicine as another 'mythic wonder drug' (Wang and Avila, 2007), there are several aspects that the clinical, scientific community and the industry are responsible for, including: (1) standardised use of terminology; (2) standardised preparation protocols; (3) standardised protocols for clinical application; (4) consistent description of source, volume, method of harvesting/processing and contents of these PPs used. Only then, further progress and breakthrough would be made in the fields of bone regeneration and tissue engineering.

\section{Conclusion}

In vitro studies in human have demonstrated the multi-faceted 'priming effect' of PPs on the biophysiological functions of BM-MSCs. PPs have been shown to improve proliferation, migration, osteogenic differentiation, immunomodulation, reaction to apoptotic stress as well as pro-angiogenic and pro-inflammatory capacities of BM-MSCs. The lack of standardised terminology and protocols surrounding the use of PPs was highlighted, along with other factors that unfortunately restrict the transferability of these findings into clinical practice. Furthermore, the impact of short-term exposure of BM-MSCs to PPs, as seen commonly in clinical practice, has not been investigated. Therefore, further collaborative multi-centre in vitro research in humans modelled to reflect clinical practice is required to better understand the effects of PP exposure on the biophysiological function(s) of BM-MSCs in human.

\section{Conflicts of interest and source of funding}

None of the authors has any conflict of interest relevant to the study and no funding was received for the completion of this project.

\section{References}

Agis H, Kandler B, Fischer MB, Watzek G, Gruber R (2009) Activated platelets increase fibrinolysis of mesenchymal progenitor cells. J Orthop Res 27: 972980.

Amable PR, Teixeira MV, Carias RB, Granjeiro JM, Borojevic R (2014) Mesenchymal stromal cell proliferation, gene expression and protein production in human platelet-rich plasma-supplemented media. PLoS One 9: e104662. DOI: 10.1371/journal. pone.0104662.

Andia I, Maffulli N (2019) New biotechnologies for musculoskeletal injuries. Surgeon 17: 244-255.

Ben Azouna N, Jenhani F, Regaya Z, Berraeis L, Ben Othman T, Ducrocq E, Domenech J (2012) Phenotypical and functional characteristics of mesenchymal stem cells from bone marrow: comparison of culture using different media supplemented with human platelet lysate or fetal bovine serum. Stem Cell Res Ther 3: 6 . DOI: $10.1186 /$ scrt97.

Bernardi M, Agostini F, Chieregato K, Amati E, Durante C, Rassu M, Ruggeri M, Sella S, Lombardi E, Mazzucato M, Astori G (2017) The production method affects the efficacy of platelet derivatives to expand mesenchymal stromal cells in vitro. J Transl Med 15: 90. DOI: 10.1186/s12967-017-1185-9.

Bernardi M, Albiero E, Alghisi A, Chieregato K, Lievore C, Madeo D, Rodeghiero F, Astori G (2013) Production of human platelet lysate by use of ultrasound for ex vivo expansion of human bone marrow-derived mesenchymal stromal cells. Cytotherapy 15: 920-929.

Bielecki T, Gazdzik TS, Szczepanski T (2008) Benefit of percutaneous injection of autologous platelet-leukocyte-rich gel in patients with delayed union and nonunion. Eur Surg Res 40: 289-296.

Bishop JA, Palanca AA, Bellino MJ, Lowenberg DW (2012) Assessment of compromised fracture healing. J Am Acad Orthop Surg 20: 273-282.

Bustos ML, Huleihel L, Kapetanaki MG, LinoCardenas CL, Mroz L, Ellis BM, McVerry BJ, Richards TJ, Kaminski N, Cerdenes N, Mora AL, Rojas M (2014) Aging mesenchymal stem cells fail to protect because of impaired migration and antiinflammatory response. Am J Respir Crit Care Med 189: 787-798. 
Calori GM, Giannoudis PV (2011) Enhancement of fracture healing with the diamond concept: the role of the biological chamber. Injury 42: 1191-1193.

Calori GM, Mazza E, Colombo M, Ripamonti C, Tagliabue L (2011) Treatment of long bone nonunions with polytherapy: indications and clinical results. Injury 42: 587-590.

Calori GM, Tagliabue L, Gala L, d'Imporzano M, Peretti G, Albisetti W (2008) Application of rhBMP-7 and platelet-rich plasma in the treatment of long bone non-unions: a prospective randomised clinical study on 120 patients. Injury 39: 1391-1402.

Chiang CC, Su CY, Huang CK, Chen WM, Chen TH, Tzeng YH (2007) Early experience and results of bone graft enriched with autologous platelet gel for recalcitrant nonunions of lower extremity. J Trauma 63: 655-661.

Choumerianou DM, Martimianaki G, Stiakaki E, Kalmanti L, Kalmanti M, Dimitriou H (2010) Comparative study of stemness characteristics of mesenchymal cells from bone marrow of children and adults. Cytotherapy 12: 881-887.

DeLong JM, Russell RP, Mazzocca AD (2012) Platelet-rich plasma: the PAW classification system. Arthroscopy 28: 998-1009.

Di Matteo B, Filardo G, Kon E, Marcacci M (2015) Platelet-rich plasma: evidence for the treatment of patellar and Achilles tendinopathy-a systematic review. Musculoskelet Surg 99: 1-9.

Dohan Ehrenfest DM, Doglioli P, de Peppo GM, Del Corso M, Charrier JB (2010) Choukroun's plateletrich fibrin (PRF) stimulates in vitro proliferation and differentiation of human oral bone mesenchymal stem cell in a dose-dependent way. Arch Oral Biol 55: 185-194.

Dohan Ehrenfest DM, Rasmusson L, Albrektsson T (2009) Classification of platelet concentrates: from pure platelet-rich plasma (P-PRP) to leucocyte- and platelet-rich fibrin (L-PRF). Trends Biotechnol 27: 158-167.

Einhorn TA, Gerstenfeld LC (2015) Fracture healing: mechanisms and interventions. Nat Rev Rheumatol 11: 45-54.

Ekegren CL, Edwards ER, de Steiger R, Gabbe BJ (2018) Incidence, costs and predictors of non-union, delayed union and mal-union following long bone fracture. Int J Environ Res Public Health 15: 2845. DOI: $10.3390 /$ ijerph15122845.

Etulain J (2018) Platelets in wound healing and regenerative medicine. Platelets 29: 556-568.

Galasso O, Mariconda M, Romano G, Capuano N, Romano L, Ianno B, Milano C (2008) Expandable intramedullary nailing and platelet rich plasma to treat long bone non-unions. J Orthop Traumatol 9: 129-134.

Ganguly P, El-Jawhari JJ, Burska AN, Ponchel F, Giannoudis PV, Jones EA (2019) The analysis of in vivo aging in human bone marrow mesenchymal stromal cells using colony-forming unit-fibroblast assay and the CD45(low)CD271(+) phenotype. Stem Cells Int 2019: 5197983. DOI: 10.1155/2019/5197983.
Ganguly P, El-Jawhari JJ, Giannoudis PV, Burska AN, Ponchel F, Jones EA (2017) Age-related changes in bone marrow mesenchymal stromal cells: a potential impact on osteoporosis and osteoarthritis development. Cell Transplant 26: 1520-1529.

Gianakos AL, Sun L, Patel JN, Adams DM, Liporace FA (2017) Clinical application of concentrated bone marrow aspirate in orthopaedics: a systematic review. World J Orthop 8: 491-506.

Giannoudis PV, Einhorn TA, Marsh D (2007) Fracture healing: the diamond concept. Injury 38 Suppl 4: S3-6.

Giannoudis PV, Einhorn TA, Schmidmaier G, Marsh D (2008) The diamond concept-open questions. Injury 39 Suppl 2: S5-8.

Goedecke A, Wobus M, Krech M, Münch N, Richter K, Hölig K, Bornhauser M (2011) Differential effect of platelet-rich plasma and fetal calf serum on bone marrow-derived human mesenchymal stromal cells expanded in vitro. J Tissue Eng Regen Med 5: 648-654.

Golos J, Walinski T, Piekarczyk P, Kwiatkowski K (2014) Results of the use of platelet rich plasma in the treatment of delayed union of long bones. Ortop Traumatol Rehabil 16: 397-406.

Gottipamula S, Sharma A, Krishnamurthy S, Majumdar AS, Seetharam RN (2012) Human platelet lysate is an alternative to fetal bovine serum for large-scale expansion of bone marrow-derived mesenchymal stromal cells. Biotechnol Lett 34: 13671374.

Gruber R, Karreth F, Kandler B, Fuerst G, Rot A, Fischer MB, Watzek G (2004) Platelet-released supernatants increase migration and proliferation, and decrease osteogenic differentiation of bone marrow-derived mesenchymal progenitor cells under in vitro conditions. Platelets 15: 29-35.

Hak DJ, Fitzpatrick D, Bishop JA, Marsh JL, Tilp S, Schnettler R, Simpson H, Alt V (2014) Delayed union and nonunions: epidemiology, clinical issues, and financial aspects. Injury 45 Suppl 2: S3-7.

Hutton B, Salanti G, Caldwell DM, Chaimani A, Schmid CH, Cameron C, Ioannidis JP, Straus S, Thorlund K, Jansen JP (2015) The PRISMA extension statement for reporting of systematic reviews incorporating network meta-analyses of health care interventions: checklist and explanations. Ann Intern Med 162: 777-784.

Infante A, Rubio-Azpeitia E, Sanchez P, Alberdi R, Rodriguez CI, Andia I (2017) Platelet rich plasma and culture configuration affect the matrix forming phenotype of bone marrow stromal cells. Tissue Eng Regen Med 14: 567-577.

Jenhani F, Durand V, Ben Azouna N, Thallet S, Ben Othmen T, Bejaoui M, Domenech J (2011) Human cytokine expression profile in various conditioned media for in vitro expansion bone marrow and umbilical cord blood immunophenotyped mesenchymal stem cells. Transplant Proc 43: 639-643.

Jonsdottir-Buch SM, Lieder R, Sigurjonsson OE (2013) Platelet lysates produced from expired 
platelet concentrates support growth and osteogenic differentiation of mesenchymal stem cells. PLoS One 8: e68984. DOI: 10.1371/journal.pone.0068984.

Karadjian M, Senger AS, Essers C, Wilkesmann S, Heller R, Fellenberg J, Simon R, Westhauser F (2020) Human platelet lysate can replace fetal calf serum as a protein source to promote expansion and osteogenic differentiation of human bone-marrow-derived mesenchymal stromal cells. Cells 9: 918. DOI: 10.3390/ cells9040918.

Kasten P, Vogel J, Beyen I, Weiss S, Niemeyer P, Leo A, Lüginbuhl R (2008) Effect of platelet-rich plasma on the in vitro proliferation and osteogenic differentiation of human mesenchymal stem cells on distinct calcium phosphate scaffolds: the specific surface area makes a difference. J Biomater Appl 23: 169-188.

Kim M, Kim C, Choi YS, Kim M, Park C, Suh Y (2012) Age-related alterations in mesenchymal stem cells related to shift in differentiation from osteogenic to adipogenic potential: implication to age-associated bone diseases and defects. Mech Ageing Dev 133: 215-225.

Kosmacheva SM, Danilkovich NN, Shchepen AV, Ignatenko SI, Potapnev MP (2014) Effect of platelet releasate on osteogenic differentiation of human mesenchymal bone marrow stem cells. Bull Exp Biol Med 156: 560-565.

Lana J, Purita J, Paulus C, Huber SC, Rodrigues BL, Rodrigues AA, Santana MH, Madureira JL, Jr., Malheiros Luzo AC, Belangero WD, AnnichinoBizzacchi JM (2017) Contributions for classification of platelet rich plasma - proposal of a new classification: MARSPILL. Regen Med 12: 565-574.

Lange C, Brunswig-Spickenheier B, Eissing L, Scheja L (2012) Platelet lysate suppresses the expression of lipocalin-type prostaglandin D2 synthase that positively controls adipogenic differentiation of human mesenchymal stromal cells. Exp Cell Res 318: 2284-2296.

Lee DH, Ryu KJ, Kim JW, Kang KC, Choi YR (2014) Bone marrow aspirate concentrate and plateletrich plasma enhanced bone healing in distraction osteogenesis of the tibia. Clin Orthop Relat Res 472: 3789-3797.

Liou JJ, Rothrauff BB, Alexander PG, Tuan RS (2018) Effect of platelet-rich plasma on chondrogenic differentiation of adipose- and bone marrow-derived mesenchymal stem cells. Tissue Eng Part A 24: 14321443.

Lucarelli E, Beccheroni A, Donati D, Sangiorgi L, Cenacchi A, Del Vento AM, Meotti C, Bertoja AZ, Giardino R, Fornasari PM, Mercuri M, Picci P (2003) Platelet-derived growth factors enhance proliferation of human stromal stem cells. Biomaterials 24: 30953100 .

Lucarelli E, Beretta R, Dozza B, Tazzari PL, O'Connel SM, Ricci F, Pierini M, Squarzoni S, Pagliaro PP, Oprita EI, Donati D (2010) A recently developed bifacial platelet-rich fibrin matrix. Eur Cell Mater 20: 13-23.
Magalon J, Chateau AL, Bertrand B, Louis ML, Silvestre A, Giraudo L, Veran J, Sabatier F (2016) DEPA classification: a proposal for standardising PRP use and a retrospective application of available devices. BMJ Open Sport Exerc Med 2: e000060. DOI: 10.1136/bmjsem-2015-000060.

Malhotra R, Kumar V, Garg B, Singh R, Jain V, Coshic P, Chatterjee K (2015) Role of autologous platelet-rich plasma in treatment of long-bone nonunions: a prospective study. Musculoskelet Surg 99: 243-248.

Mariconda M, Cozzolino F, Cozzolino A, D'Agostino E, Bove A, Milano C (2008) Platelet gel supplementation in long bone nonunions treated by external fixation. J Orthop Trauma 22: 342-345.

Martin JR, Houdek MT, Sierra RJ (2013) Use of concentrated bone marrow aspirate and platelet rich plasma during minimally invasive decompression of the femoral head in the treatment of osteonecrosis. Croat Med J 54: 219-224.

Marx RE (2001) Platelet-rich plasma (PRP): what is PRP and what is not PRP? Implant Dent 10: 225-228.

Marx RE (2004) Platelet-rich plasma: evidence to support its use. J Oral Maxillofac Surg 62: 489-496.

Mautner K, Malanga GA, Smith J, Shiple B, Ibrahim V, Sampson S, Bowen JE (2015) A call for a standard classification system for future biologic research: the rationale for new PRP nomenclature. PM R 7: S53-S59.

Mills LA, Simpson AH (2013) The relative incidence of fracture non-union in the Scottish population (5.17 million): a 5-year epidemiological study. BMJ Open 3: e002276. DOI: 10.1136/bmjopen-2012-002276.

Mishra A, Harmon K, Woodall J, Vieira A (2012) Sports medicine applications of platelet rich plasma. Curr Pharm Biotechnol 13: 1185-1195.

Moher D, Liberati A, Tetzlaff J, Altman DG (2009) Preferred reporting items for systematic reviews and meta-analyses: the PRISMA statement. Ann Intern Med 151: 264-269.

Moisley KM, El-Jawhari JJ, Owston H, Tronci G, Russell SJ, Jones EA, Giannoudis PV (2019) Optimising proliferation and migration of mesenchymal stem cells using platelet products: a rational approach to bone regeneration. J Orthop Res 37: 1329-1338.

Moradian H, Rafiee A, Ayatollahi M (2017) Design and fabrication of a novel transplant combined with human bone marrow mesenchymal stem cells and platelet-rich fibrin: new horizons for periodontal tissue regeneration after dental trauma. Iran J Pharm Res 16: 1370-1378.

Muraglia A, Ottonello C, Spanò R, Dozin B, Strada P, Grandizio M, Cancedda R, Mastrogiacomo M (2014) Biological activity of a standardized freezedried platelet derivative to be used as cell culture medium supplement. Platelets 25: 211-220.

Muraglia A, Todeschi MR, Papait A, Poggi A, Spanò R, Strada P, Cancedda R, Mastrogiacomo M (2015) Combined platelet and plasma derivatives enhance proliferation of stem/progenitor cells maintaining their differentiation potential. Cytotherapy 17: 17931806. 
Murphy MB, Blashki D, Buchanan RM, Yazdi IK, Ferrari M, Simmons PJ, Tasciotti E (2012) Adult and umbilical cord blood-derived platelet-rich plasma for mesenchymal stem cell proliferation, chemotaxis, and cryo-preservation. Biomaterials 33: 5308-5316.

Nguyen ATM, Tran HLB, Pham TAV (2019) In vitro evaluation of proliferation and migration behaviour of human bone marrow-derived mesenchymal stem cells in presence of platelet-rich plasma. Int J Dent 2019: 9639820. DOI: 10.1155/2019/9639820.

Parsons P, Butcher A, Hesselden K, Ellis K, Maughan J, Milner R, Scott M, Alley C, Watson JT, Horner A (2008) Platelet-rich concentrate supports human mesenchymal stem cell proliferation, bone morphogenetic protein-2 messenger RNA expression, alkaline phosphatase activity, and bone formation in vitro: a mode of action to enhance bone repair. J Orthop Trauma 22: 595-604.

Perut F, Filardo G, Mariani E, Cenacchi A, Pratelli L, Devescovi V, Kon E, Marcacci M, Facchini A, Baldini N, Granchi D (2013) Preparation method and growth factor content of platelet concentrate influence the osteogenic differentiation of bone marrow stromal cells. Cytotherapy 15: 830-839.

Prins HJ, Rozemuller H, Vonk-Griffioen S, Verweij VG, Dhert WJ, Slaper-Cortenbach IC, Martens AC (2009) Bone-forming capacity of mesenchymal stromal cells when cultured in the presence of human platelet lysate as substitute for fetal bovine serum. Tissue Eng Part A 15: 3741-3751.

Roffi A, Di Matteo B, Krishnakumar GS, Kon E, Filardo G (2017) Platelet-rich plasma for the treatment of bone defects: from pre-clinical rational to evidence in the clinical practice. A systematic review. Int Orthop 41: 221-237.

Rombouts WJ, Ploemacher RE (2003) Primary murine MSC show highly efficient homing to the bone marrow but lose homing ability following culture. Leukemia 17: 160-170.

Samuel S, Ahmad RE, Ramasamy TS, Karunanithi P, Naveen SV, Murali MR, Abbas AA, Kamarul T (2016) Platelet-rich concentrate in serum free medium enhances osteogenic differentiation of bone marrowderived human mesenchymal stromal cells. PeerJ 2016 4: e2347. DOI: 10.7717/peerj.2347.

Sanchez M, Anitua E, Cugat R, Azofra J, Guadilla J, Seijas R, Andia I (2009) Nonunions treated with autologous preparation rich in growth factors. J Orthop Trauma 23: 52-59.

Say F, Turkeli E, Bulbul M (2014) Is platelet-rich plasma injection an effective choice in cases of nonunion? Acta Chir Orthop Traumatol Cech 81: 340345.

Schallmoser K, Henschler R, Gabriel C, Koh MBC, Burnouf T (2020) Production and quality requirements of human platelet lysate: a position statement from the working party on cellular therapies of the International Society of Blood Transfusion. Trends Biotechnol 38: 13-23.

Schar MO, Diaz-Romero J, Kohl S, Zumstein MA, Nesic D (2015) Platelet-rich concentrates differentially release growth factors and induce cell migration in vitro. Clin Orthop Relat Res 473: 1635-1643.

Scherer SS, Tobalem M, Vigato E, Heit $Y$, Modarressi A, Hinz B, Pittet B, Pietramaggiori G (2012) Nonactivated versus thrombin-activated platelets on wound healing and fibroblast-tomyofibroblast differentiation in vivo and in vitro. Plast Reconstr Surg 129: 46e-54e.

Skific M, Golemovic M, Crkvenac-Gornik K, Vrhovac R, Golubic Cepulic B (2018) Comparative analysis of biological and functional properties of bone marrow mesenchymal stromal cells expanded in media with different platelet lysate content. Cells Tissues Organs 205: 226-239.

Soares CS, Babo PS, Reis RL, Carvalho PP, Gomes ME (2020) Platelet-derived products in veterinary medicine: a new trend or an effective therapy? Trends Biotechnol. DOI: 10.1016/j.tibtech.2020.07.011.

Spees JL, Gregory CA, Singh H, Tucker HA, Peister A, Lynch PJ, Hsu SC, Smith J, Prockop DJ (2004) Internalized antigens must be removed to prepare hypoimmunogenic mesenchymal stem cells for cell and gene therapy. Mol Ther 9: 747-756.

Su P, Tian Y, Yang C, Ma X, Wang X, Pei J, Qian A (2018) Mesenchymal stem cell migration during bone formation and bone diseases therapy. Int J Mol Sci 19: 2343. DOI: 10.3390/ijms19082343.

Verboket R, Leiblein M, Seebach C, Nau C, Janko M, Bellen M, Bonig H, Henrich D, Marzi I (2018) Autologous cell-based therapy for treatment of large bone defects: from bench to bedside. Eur J Trauma Emerg Surg 44: 649-665.

Verrier S, Meury TR, Kupcsik L, Heini P, Stoll T, Alini M (2010) Platelet-released supernatant induces osteoblastic differentiation of human mesenchymal stem cells: potential role of BMP-2. Eur Cell Mater 20: 403-414.

Vogel JP, Szalay K, Geiger F, Kramer M, Richter W, Kasten P (2006) Platelet-rich plasma improves expansion of human mesenchymal stem cells and retains differentiation capacity and in vivo bone formation in calcium phosphate ceramics. Platelets 17: 462-469.

Wang HL, Avila G (2007) Platelet rich plasma: myth or reality? Eur J Dent 1: 192-194.

Wang T, Zhang X, Bikle DD (2017) Osteogenic differentiation of periosteal cells during fracture healing. J Cell Physiol 232: 913-921.

Yin W, Qi X, Zhang Y, Sheng J, Xu Z, Tao S, Xie X, Li X, Zhang C (2016) Advantages of pure platelet-rich plasma compared with leukocyte- and platelet-rich plasma in promoting repair of bone defects. J Transl Med 14: 73. DOI: 10.1186/s12967-016-0825-9.

\section{Discussion with Reviewer}

Reviewer: In addition to the variations due to many preparation protocols, it would be interesting to have the authors' opinion on the inter-individual variations. Prepared in similar conditions, PPs from 
different patients will have different properties (in term of efficacy). Do you think that the preparation of PPs has more influence on its outcome than the patient of origin?

Authors: Factors affecting BM-MSC functions are multifactorial. Both PP preparation method and donor play equally important roles in determining the outcomes when using PPs clinically to optimise BM-MSC function. However, whilst patient's biology is often not-modifiable, the choice of PPs and its components used (e.g. only growth factors in PL/ PR; combination of both cells and growth factors in PRP) as well as its preparation and incubation period with BM-MSCs are all flexible and remains at the clinician's disposal when treating patients with autologous PPs.

Editor's note: The Scientific Editor responsible for this paper was Martin Stoddart. 